NATIONAL INSTITUTE OF STANDARDS

AND TECHNOLOGY

NIST Technical Note 1318

\title{
Variances Based on Data With Dead Time Between the Measurements
}

James A. Barnes

David W. Allan 



\section{Variances Based on Data With Dead Time Between the Measurements}

James A. Barnest

David W. Allan

Time and Frequency Division

Center for Basic Standards

Natlonal Measurement Laboratory

Natlonal Institute of Standards and Technology

Boulder, Colorado 80303-3328

†Austron, Inc.

Boulder, Colorado 80301

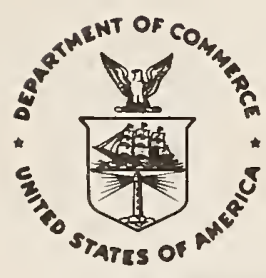

U.S. DEPARTMENT OF COMMERCE, Robert A. Mosbacher, Secretary NATIONAL INSTITUTE OF STANDARDS AND TECHNOLOGY. John W Lvons, Director 
National Institute of Standards and Technology Technical Note 1318 Natl. Inst. Stand. Technol., Tech. Note 1318, 48 pages (Mar. 1990) CODEN:NTNOEF

U.S. GOVERNMENT PRINTING OFFICE WASHINGTON: 1990 


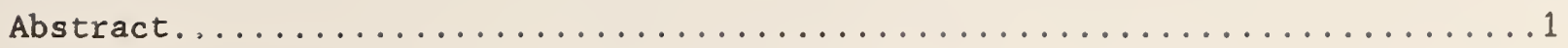

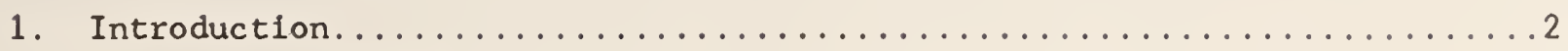

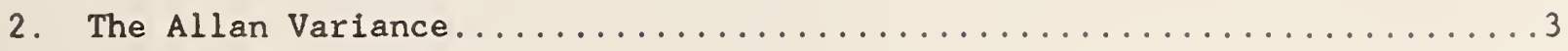

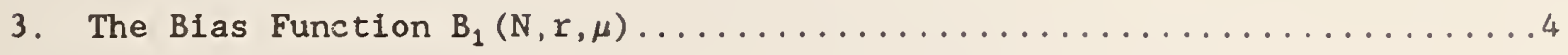

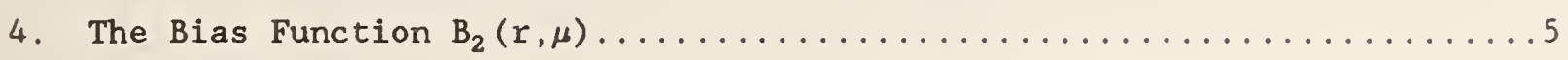

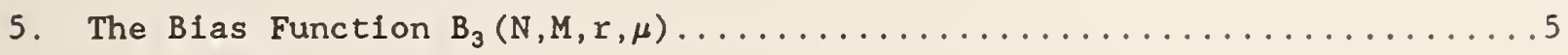

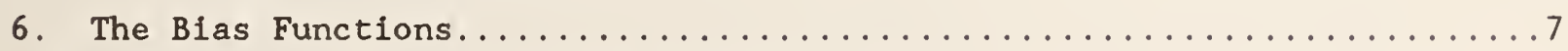

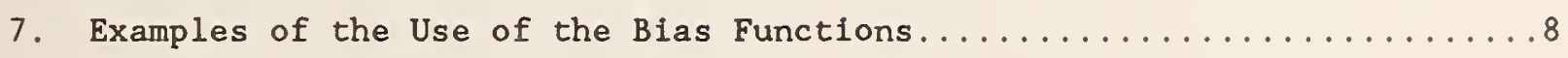

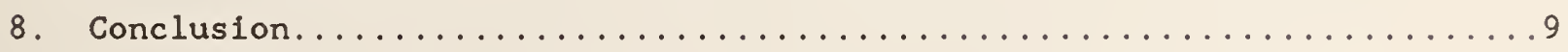

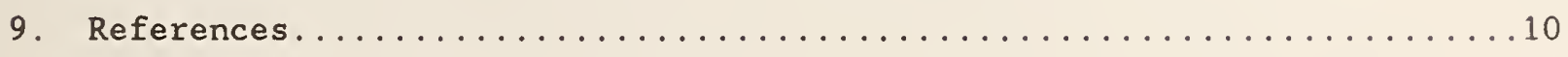

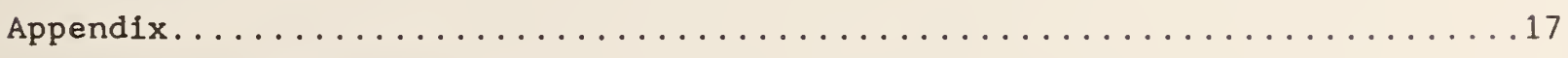

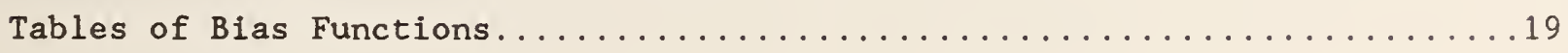



VARIANCES BASED ON DATA WITH DEAD TIME BETWEEN THE MEASUREMENTS

\author{
James A. Barnes \\ Austron, Inc. \\ Boulder, Colorado 80301 \\ and \\ David W. Allan \\ Time and Frequency Division \\ National Institute of Standards and Technology \\ Boulder, Colorado 80303
}

The accepted definition of frequency stability in the time domain is the two-sample variance (or Allan variance). It is based on the measurement of average frequencies over adjacent time intervals, with no "dead time" between the intervals. The primary advantages of the Allan variance are that (1) it is convergent for many encountered noise models for which the conventional variance is divergent; (2) it can distinguish between many important and different spectral noise types; (3) the two-sample approach relates to many practical implementations; for example, the rms change of an oscillator's frequency from one period to the next; and (4) Allan variances can be easily estimated at integer multiples of the sample interval.

In 1974 a table of bias functions which related variance estimates with various configurations of number of samples and dead time to the Allan variance was published [1]. The tables were based on noises with pure power-law spectral densities.

Often situations occur that unavoidably have dead time between measurements, but still the conventional variances are not

convergent. Some of these applications are outside of the time-andfrequency field. Also, the dead times are often distributed throughout a given average, and this distributed dead time is not treated in the 1974 tables.

This paper reviews the bias functions $B_{1}(N, r, \mu)$, and $B_{2}(r, \mu)$ and introduces a new bias function, $B_{3}(2, M, r, \mu)$, to handle the commonly occurring cases of the effect of distributed dead time on the computed variances: Some convenient and easy-to-interpret asymptotic limits are reported. A set of tables for the bias functions are included at the end of this paper.

Key words: Allan variance; bias functions; data sampling and dead time; dead time between the measurement; definition of frequency stability; distributed dead time; two-sample variance 


\section{Introduction}

The sample mean and variance indicate respectively the approximate magnitude of a quantity and its uncertainty. For many situations a continuous function of time is sampled, or measured, at fairly regular intervals. Sampling is not always instantaneous. It takes a finite time and provides an "average reading." If the underlying process (or noise) is random and uncorrelated in time, then the fluctuations are said to be "white" noise. In this situation, the sample mean and variance calculated by the conventional formulas,

$$
\begin{aligned}
& m=\frac{1}{N} \sum_{n=1}^{N} \bar{y}_{n}, \\
& s^{2}=\frac{1}{N-1} \sum_{n=1}^{N}\left(\bar{y}_{n}-m\right)^{2},
\end{aligned}
$$

provide the needed information. The "bar" over the y in eq (1) above denotes the average over a finite time interval. In time and frequency work, $y$ is defined as the average fractional (or normalized) frequency deviation from nominal over an interval $\tau$ and at some specified measurement time. As in science generally, the physical model determines the appropriate mathematical model. For the white noise model, the sample mean and variance are the mainstays of most analyses.

Although white noise is a common model for many physical processes, more general noise models are being identified and used. In precise time and frequency measurement, for example, there are two quantities of great interest: instantaneous frequency and phase. These two quantities by definition are exactly related by a differential. (We are NOT considering Fourier frequencies at this point.) That is, the instantaneous frequency is the time rate of change of phase. Thus, if we were employing a model of white frequency-modulation (white FM) noise, then the phase noise is the integral of the white FM noise, commonly called a Brownian motion or random walk. Therefore, depending on whether we are currently interested in phase or frequency, the sample mean and variance may or may not be appropriate. 
By definition, white noise has a power spectral density (PSD) that is constant with Fourier frequency. Since random walk noise is the integral of white noise, the power spectral density of a random walk varies as $1 / f^{2}$ (where $f$ is the Fourier frequency) [2]. We encounter noise models whose power spectral densities are various power laws of their Fourler frequencles. Flicker noise is very common and is defined as a nolse whose power spectral density varies as $1 / \mathrm{f}$ over a relevant spectral range. If an oscillator's instantaneous frequency is well modeled by flicker noise, then its phase would be the integral of the flicker noise. It would have a PSD which varied as $1 / f^{3}$.

Nolse models whose PSD's are power laws of the Fourler frequency but not integer exponents are possible as well but not as common. This paper considers power-law PSD's of a quantity $y(t) ; y(t)$ is a continuous sample function which can be measured at regular intervals. For noises whose PSD's vary as $f^{\alpha}$ with $\alpha<-1$ at low frequencles, the conventional sample mean and variance given in eq (1) do not converge as $\mathrm{N}$ gets large [2, 3]. This lack of convergence renders the sample mean and variance ineffective and often misleading in some situations.

Although the sample mean and variance have limitations, other time-domain statistics can be convergent and quite useful. The quantities that we consider in this paper depend significantly on the details of the sampling procedures. Indeed, each sampling scheme has its own bias, and this is the motivation for the bias functions discussed in this paper.

\section{The Allan Variance}

Recognizing that for particular types of noise, the conventional sample variance fails to converge as the number of samples, $N$, grows, Allan suggested that we set $N=2$ and average many of these two-sample variances to get a convergent and stable measure of the spread of the quantity in question [3]. This is what has come to be called the Allan variance. 
More specifically let us consider a sample function of time as indicated in IIgure 1. A measurement consists of averaging $y(t)$ over the interval $r$. The next measurement begins at a time $T$ after the beginning of the previous measurement interval. There is no logical reason why $T$ must be as large as $T$ or larger--if $T<r$, then the second measurement begins before the first is completed, which is unusual but possible. When $T=r$, there is no dead time between measurements.

The accepted definition of the Allan variance is the expected value of a twosample varlance with no dead time between successive measurements. In symbols, the Allan variance is given by

$$
\sigma_{y}^{2}(\tau)=3 / 2 E\left[\left(\bar{y}_{n+1}-\bar{y}_{n}\right)^{2}\right]
$$

where there is no dead time between the two sample averages for the Allan variance and the $E[\cdot]$ denotes the expectation operator.

\section{The Bias Function $B_{1}(N, \Gamma, \mu)$}

Define $N$ to be the number of sample averages of $y(t)$ used in eq (1) to estimate a sample variance ( $N=2$ for an Allan variance). Also define $r$ to be the ratio of $\mathrm{T}$ to $\tau$ ( $r=1$ when there is no dead time between measurements). The parameter $\mu$ is related to the exponent of the power law of the PSD of the process $y(t)$. If $\alpha$ is the exponent in the power-law spectrum for $y(t)$, then the Allan variance varies as $\tau$ raised to the $\mu$ power, where $\alpha$ and $\mu$ are related as shown in figure 2 [2-4]. We can use estimates of $\mu$ to infer $\alpha$, the spectral type. The ambiguity in $\alpha$ for $\mu=-2$ has been resolved by using a modified $\sigma_{\mathrm{y}}^{2}(\tau)[5-7]$.

Often data cannot be taken without dead time between sample averages, and it is useful to consider other than two-sample variances. We will define the bias function $B_{1}(N, r, \mu)$ by the ratio,

$$
B_{1}(N, r, \mu)=\frac{\sigma^{2}(N, T, \tau)}{\sigma^{2}(2, T, \tau)}
$$


where $\sigma^{2}(\mathrm{~N}, \mathrm{~T}, \tau)$ is the expected sample variance given in eq (1) and based on $\mathrm{N}$ measurements at intervals $\mathrm{T}$ and averaged over a time $\tau$ and $\mathrm{r}=\mathrm{T} / \tau$. In words, $B_{1}(N, r, \mu)$ is the ratio of the expected variance for $N$ measurements to the expected variance for two samples (everything else held constant). The variances on the right in eq (3) depend implicitly on the noise type even though $\mu$ or $\alpha$ are not shown as independent variables. The noise-type parameter, $\mu$, is shown as an independent variable for all of the bias functions in this paper, because the values of the ratio of these variances explicitly depend on $\mu$ as will be derived later in the paper. Allan showed that if $\mathrm{N}$ and $\mathrm{r}$ are held constant, then the $\alpha, \mu$ relationship shown in figure 2 is the same; that is, we can still infer the spectral type from the $\tau$ dependence using the equation $\alpha=-\mu-1,-2 \leq \mu<2$ [3] .

\section{The Bias Function $B_{2}(r, \mu)$}

The bias function $B_{2}(r, \mu)$ is defined in [1] by the relation,

$$
\mathrm{B}_{2}(r, \mu)=\frac{\sigma^{2}(2, T, \tau)}{\sigma^{2}(2, \tau, \tau)}=\frac{\sigma^{2}(2, T, \tau)}{\sigma_{y}^{2}(\tau)} .
$$

In words, $B_{2}(r, \mu)$ is the ratio of the expected two-sample variance with dead time to that without dead time (with $N=2$ and $\tau$ the same for both variances). A plot of the $B_{2}(r, \mu)$ function is shown in figure 3 . The bias functions $B_{1}$ and $B_{2}$ represent biases relative to $N=2$ rather than infinity; that is, the ratio of the $N$ sample variance (with or without dead time) to the Allan variance and the ratio of the two-sample dead-time variance to the Allan variance respectively.

\section{The Bias Function $B_{3}(N, M, r, \mu)$}

Consider the case where a great many measurements are available with dead time between each pair of measurements $\left(T_{0}>\tau_{0}\right)$. The measurements are averaged over the time interval $\tau_{0}$, the spacing between the beginning of one measurement to the next is $\mathrm{T}_{0}$, and it may not be convenient to retake the data. We might want to estimate the Allan variance at, say, multiples $M$ of 
the averaging time $\tau_{0}$. If we average groups of the measurements of $y(t)$, then the dead times between the original measurements are distributed periodically throughout the new average measurements (see figure 4). Define

$$
\overline{\bar{y}}_{1}=\frac{1}{M} \sum_{n=i}^{M+i-1} \bar{y}_{n} \text {, }
$$

where $\bar{y}_{1}$ are the raw or original measurements based on dead time $T_{0}-\tau_{0}$.

Also define the two-sample variance with distributed dead time as

$$
\sigma^{2}(2, M, T, \tau)=\frac{1}{2} E\left[\left(\overline{\bar{y}}_{1}-\overline{\bar{y}}_{1+M}\right)^{2}\right]
$$

with $\tau=\mathrm{M} \tau_{0}$ and $\mathrm{T}=\mathrm{MT}_{0}$.

We can now define $B_{3}$ as the ratio of the $\mathrm{N}$-sample variance with distributed dead time to the $\mathrm{N}$-sample variance with dead time accumulated at the end as in figure 1:

$$
B_{3}(N, M, r, \mu)=\frac{\sigma^{2}(N, M, T, \tau)}{\sigma^{2}(N, T, \tau)}
$$

Although $B_{3}(N, M, r, \mu)$ is defined for general $N$, the tables in the Appendix confine treatment to the case where $N=2$. There is little value in extending the tables to include general $\mathrm{N}$. Though the variances on the right in eq (7) depend explicitly on $N, T$ and $\tau$, the ratio $B_{3}(N, M, r, \mu)$ depends on the ratio $\mathrm{r}=\mathrm{T} / \tau$, and on $\mu$ as developed later in this paper.

In words, $B_{3}(2, M, r, \mu)$ is the ratio of the expected two-sample variance with periodically distributed dead time, as shown in figure 4, to the expected twosample variance with all the dead time grouped together as shown in figure 1. Both the numerator and the denominator have the same total averaging time and dead time, but they are apportioned differently. The product $B_{2}(r, \mu) \cdot$ $B_{3}(2, M, r, \mu)$ is the distributed dead-time variance over the Allan variance for a particular $\mathrm{T}, \tau, \mathrm{M}$ and $\mu$. 
Some useful asymptotic forms of $B_{3}$ can be found. In the case of large $M$ and $M>r$, we may write that

$$
\begin{array}{ll}
\mathrm{B}_{3} \simeq \frac{1+\mu}{3}, & 1 \leq \mu \leq 2, \\
\mathrm{~B}_{3} \simeq \frac{4 \ln (2)}{2 \ln (r)+3}, & \mu=0 .
\end{array}
$$

One simple and important conclusion from these two equations is that for the cases of flicker FM noise and random-walk FM noise, the $\tau^{\mu}$ dependence for large, is the same whether or not there is periodically distributed dead time. The values of the variances differ only by a constant, and in the latter case the constant is 1 . This conclusion is also true for white FM noise, and in this case the constant is also 1.

In the cases $r \gg 1$ and $-2 \leq \mu \leq-1$, we may write for the asymptotic behavior of $\mathrm{B}_{3}$

$$
B_{3} \simeq M^{\alpha}, \alpha=-\mu-1
$$

as was determined empirically. In this region of power-law spectrum the $B_{3}$ function has an $M^{\alpha}$ dependence for an $f^{\alpha}$ spectrum.

\section{The Bias Functions}

The bias functions can be written fairly simply by first defining the function,

$$
F(A)=2 A^{\mu+2}-(A+1)^{\mu+2}-|(A-1)|^{\mu+2} .
$$

The bias functions become

$$
B_{1}(N, r, \mu)=\frac{1+\sum_{n=1}^{N-1} \frac{N-n}{N(N-1)} \cdot F(n r)}{1+\frac{1}{2} F(r)},
$$




$$
B_{2}(r, \mu)=\frac{1+3 / 2 F(r)}{2\left(1-2^{\mu}\right)}
$$

as given in [1], and

$B_{3}(2, M, r, \mu)=\frac{2 M+M \cdot F(M r)-\sum_{n=1}^{M-1}(M-n)[2 F(n r)-F((M+n) r)-F((M-n) r)]}{\left(M^{\mu+2}\right)[F(r)+2]}$,

as indicated in the appendix.

For $\mu=0$, eqs (11), (12), and (13) are the indeterminate form $0 / 0$ and must be evaluated by l'Hôpital's rule. Special attention must also be given when expressions of the form $0^{\circ}$ arise. We verified a random sampling of the table entries using noise simulation and Monte Carlo techniques. No errors were detected. The results in this paper differ some from those in [8], which suggests that there may be some mistakes. Tables for the three bias functions are listed at the end of the paper (note that the computer print-out did not have a symbol for Greek $m u \equiv \mu$ ).

\section{Examples of the Use of the Bias Functions}

The spectral type, that is, the value of $\mu$, may be inferred by varying $\tau$, the sample time. However, another useful way of determining the value of $\mu$ is by using $B_{1}(N, r, \mu)$ as follows: calculate an estimate of $\sigma_{y}^{2}(N, T, \tau)$ and $\sigma_{y}^{2}(2, T, \tau)$ and hence $B_{1}(N, r, \mu)$; then use the tables to infer the value of $\mu$.

Suppose one has an experimental value for $\sigma_{\mathrm{y}}^{2}\left(\mathrm{~N}_{1}, \mathrm{~T}_{1}, \tau_{1}\right)$ and its spectral type is known, that is, $\mu$ is known. Suppose also that one wishes to know the variance at some other set of measurement parameters, $\mathrm{N}_{2}, \mathrm{~T}_{2}, \tau_{2}$. An unbiased estimate of $\sigma_{\mathrm{y}}^{2}\left(\mathrm{~N}_{2}, \mathrm{~T}_{2}, \tau_{2}\right)$ may be calculated by the equation:

$$
\sigma_{\mathrm{y}}^{2}\left(\mathrm{~N}_{2}, \mathrm{~T}_{2}, \tau_{2}\right)=\left(\frac{\tau_{2}}{\tau_{1}}\right)^{\mu}\left(\frac{\mathrm{B}_{1}\left(\mathrm{~N}_{2}, \mathrm{r}_{2}, \mu\right) \mathrm{B}_{2}\left(\mathrm{r}_{2}, \mu\right)}{\mathrm{B}_{1}\left(\mathrm{~N}_{1}, \mathrm{r}_{1}, \mu\right) \mathrm{B}_{2}\left(\mathrm{r}_{1}, \mu\right)}\right) \sigma_{\mathrm{y}}^{2}\left(\mathrm{~N}_{1}, \mathrm{~T}_{1}, \tau_{1}\right)
$$

where $r_{1}=T_{1} / \tau_{1}$ and $r_{2}=T_{2} / \tau_{2}$. 
Since the time-domain definition for frequency stability is the Allan variance, it behooves us, where possible, to relate other variances to the Allan variance. If we have an $\mathrm{N}$-sample variance on data with dead-time $\mathrm{T}-\tau$ and we know the power-law spectral type (the value of $\mu$ ), then we may write

$$
\sigma_{y}^{2}(\tau)=\frac{\sigma_{y}^{2}(N, T, \tau)}{B_{1}(\mathrm{~N}, \mathrm{r}, \mu) \mathrm{B}_{2}(\mathrm{r}, \mu)} .
$$

If we have an $\mathrm{N}$-sample variance where each data entry is an average of $M$ samples with distributed dead time, then we may write

$$
\sigma_{\mathrm{y}}^{2}(\tau)=\frac{\sigma_{\mathrm{y}}^{2}(\mathrm{~N}, \mathrm{M}, \mathrm{T}, \tau)}{\mathrm{B}_{1}(\mathrm{~N}, \mathrm{r}, \mu) \mathrm{B}_{2}(\mathrm{r}, \mu) \mathrm{B}_{3}(\mathrm{~N}, \mathrm{M}, \mathrm{r}, \mu)} .
$$

\section{Conclusion}

For some important power-law spectral density models often used in characterizing precision oscillators $\left(S_{y}(f) \sim f^{\alpha}, \alpha=-2,-1,0,+1,+2\right)$, we have studied the effects on variances when there is dead time between the frequency samples, and the frequency samples are averaged to increase the integration time. Since dead time between measurements is a common problem throughout metrology, the analysis here has broader applicability than just to time and frequency. Specifically, this kind of analysis has been used with gage blocks and standard volt cells--showing that the classical variance may be non-convergent in some cases [9].

Heretofore, the Allan variance has been shown to have some convenient theoretical properties in relation to power-law spectra as the integration or sample time is varied (if $\sigma_{y}{ }^{2}(\tau) \sim \tau^{\mu}$, then $\alpha=-\mu-1,-2<\mu \leq 2$ ). Since $\sigma_{y}(\tau)$, by definition, is estimated from data with no dead time, the sample or integration time can be unambiguously changed to investigate the $\tau$ dependence. From our analysis, we have concluded that for the asymptotic limit of several samples being averaged with dead time present in the data, the $\tau$ dependence of the variances is the same. The $\alpha=-\mu-1$ relationship still remains valid for white FM noise $(\mu=-1, \alpha=0)$, flicker FM noise $(\mu=0, \alpha=-1)$, and for 
random-walk FM noise $(\mu=+1, \alpha=-2)$. The asymptotic limit is approached as the product of number of samples averaged and the initial data sample time, $\tau_{0}$, becomes larger than the dead time $(M>r)$. The variances so obtained differ only by a constant, which can be calculated as given in this paper.

A knowledge of the appropriate power-law spectral model is required to translate a distributed dead-time variance to the corresponding value of the Allan variance. In principle, the power-law spectral model can be estimated from the $\tau^{\mu}$ dependence, using the variance analysis on the data as outlined above.

9. References

[1] J.A. Barnes, "Tables of Bias Functions, $B_{1}$ and $B_{2}$, for Variances Based on Finite Samples of Processes with Power Law Spectral Densities," NBS Tech. Note 375 (1969).

[2] J.A. Barnes, "Atomic Timekeeping and the Statistics of Precision Signal Generation," IEEE Proc. 54, No. 2, pp. 207-220, Feb. 1966.

[3] D.W. Allan, "Statistics of Atomic Frequency Standards, "IEEE Proc. 54, No. 2, pp. 221-230, Feb. 1966.

[4] RFC Vessot, L. Mueller, and J. Vanier, "The Specification of Oscillator Characteristics from Measurements Made in the Frequency Domain," IEEE Proc. 54, No. 2, pp. 199-207, Feb. 1966.

[5] D.W. Allan and J.A. Barnes, "A Modified "Allan Variance" With Increased Oscillator Characterization Ability," Proc. 35th Annual Frequency Control Symposium, USAERADCOM, Ft. Monmouth, NJ, May 1981 , pp. 470-475.

[6] D.W. Allan, "Time and Frequency (Time-Domain) Characterization, Estimation, and Prediction of Precision Clocks and Oscillators," IEEE Transactions on UFFC, November 1987.

[7] P. Lesage and T. Ayi, "Characterization of Frequency Stability: Analysis of the Modified Allan Variance and Properties of its Estimate," IEEE Trans. Instrum. Meas., IM-33, no. 4, pp. 332-336, Dec. 1984.

[8] N.D. Faulkner and E.V.I. Mestre, "Time-Domain Analysis of Frequency 
Stability Using Non-zero Dead-Time Counter Techniques," IEEE Trans. on Instrum. \& Meas., IM-34, 144-151 (1985).

[9] D.W. Allan, "Should the Classical Varlance Be Used as a Basic Measure in Standards Metrology?" IEEE Trans. on Instrumentation and Measurement, IM-36, 646-654, 1987 . 
Table 1. Table of some bias function identities

$$
\begin{array}{ll}
B_{1}(2, r, \mu) & =1 \\
B_{1}(N, r, 2) & =(N(N+1)) / 6 \\
B_{1}(N, 1,1) & =N / 2 \\
B_{1}(N, 1, \mu) & =\left(N\left(1-N^{\mu}\right)\right) /\left[\left(2(N-1)\left(1-2^{\mu}\right)\right] \text { for } \mu \neq 0\right. \\
& =N \text { for } \mu=0 \\
B_{1}(N, 1, \mu) & =1 \text { for } \mu<0 \\
& =\left[2 /(N(N-1)] \sum_{n=1}^{N-1}(N-n) \cdot n^{\mu} \text { for } \mu>0\right. \\
B_{1}(N, r,-1) & =1 \text { if } r \geq 1 \\
B_{1}(N, r,-2) & =1 \text { if } r \neq 1 \text { or } 0 \\
B_{2}(0, \mu) & =0 \\
B_{2}(1, \mu) & =1 \\
B_{2}(r, 2) & =r^{2} \\
B_{2}(r, 1) & =(3 r-1) / 2 \text { if } r \geq 1 \\
B_{2}(r,-1) & =r \text { if } 0 \leq r \leq 1 \\
B_{2}(r,-2) & =1 \text { if } r \geq 1 \\
& =0 \text { if } r=0 \\
B_{3}(2, M, 1, \mu) & =1 \text { if } r=1 \\
B_{3}(2, M, r,-2) & =M \\
B_{3}(2, r, \mu) & =1 \\
B_{3}(2, M, r, 2) & =1 \\
B_{3}(2, M, r,-1) & =1 \text { for } r \geq 1 \\
& =1 \\
&
\end{array}
$$




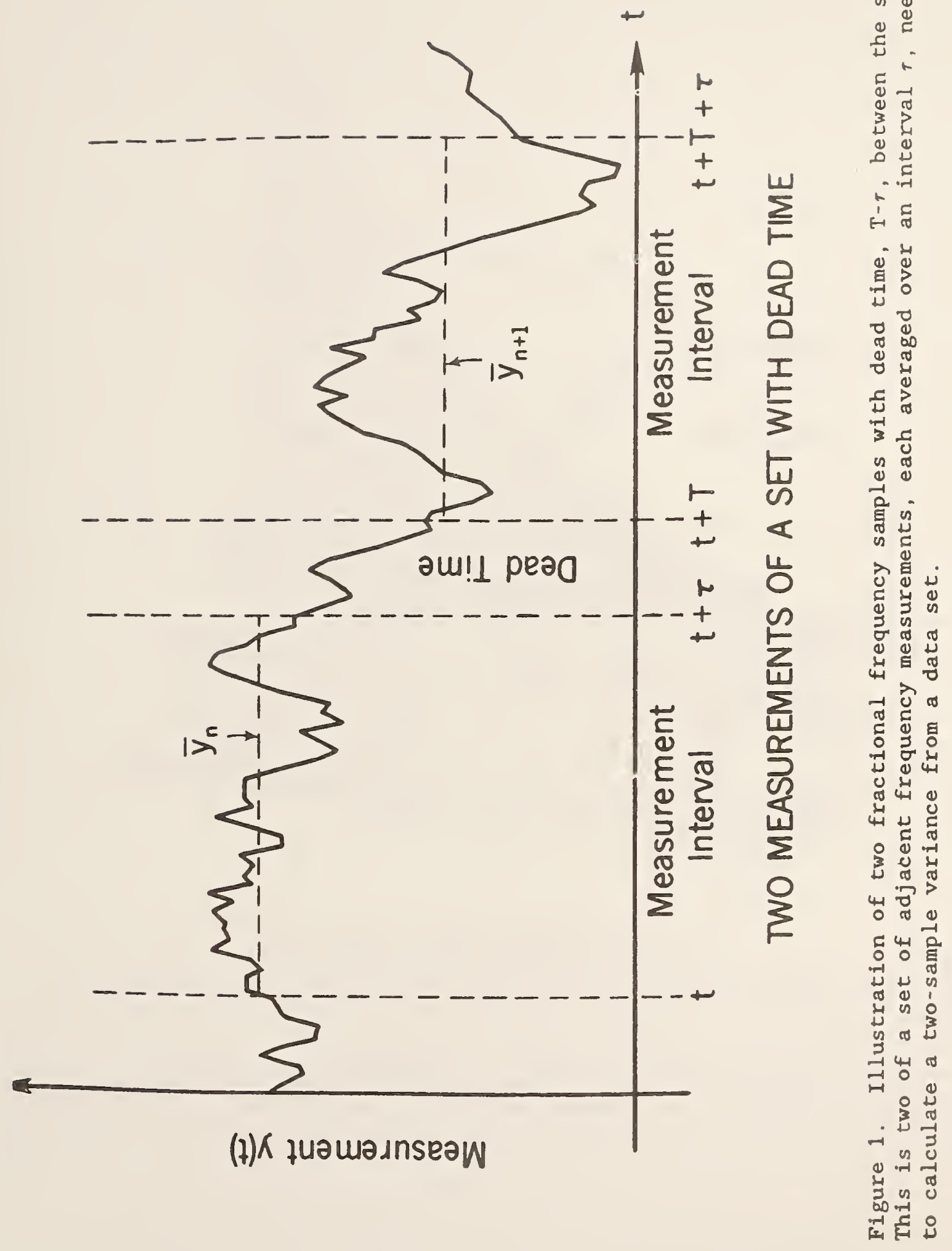




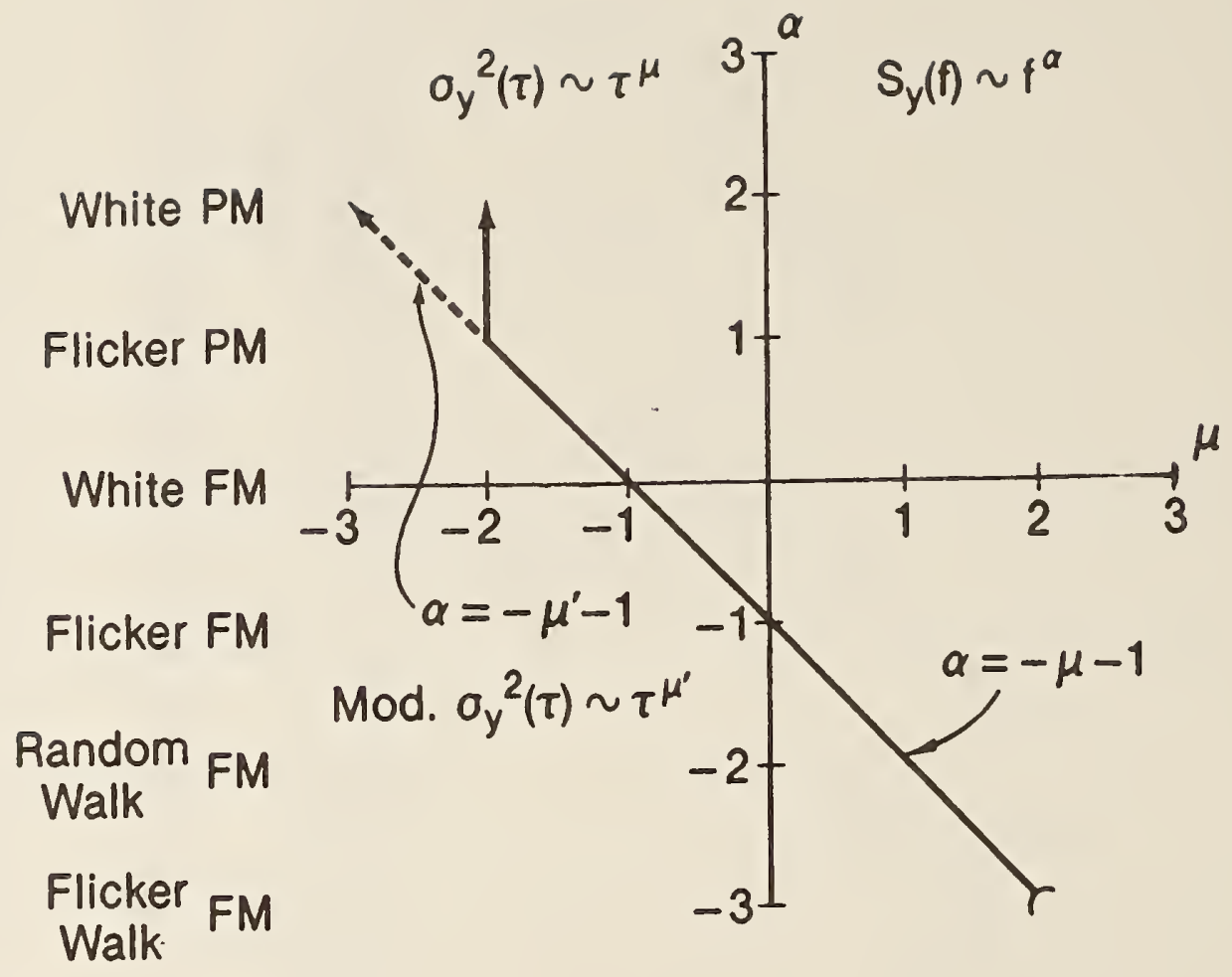

Figure 2. A plot of the relationship between the frequency-domain power-law spectral-density exponent $\alpha$ and the time-domain two-sample Allan variance exponent $\mu(\alpha=-\mu-1,-2 \leq \mu<2$ and $\alpha \geq 1$ for $\mu=-2)$. Also shown is the similar relationship between $\alpha$ and the modified Allan variance with exponent on $\tau$ of $\mu^{\prime}\left(\alpha=-\mu^{\prime}-1,-4 \leq \mu^{\prime}<2\right)$. The pointing arrows indicate the mualpha relationship ( $\alpha$ vs. $\mu$ or $\mu^{\prime}$ ) for which the particular variance applies. 


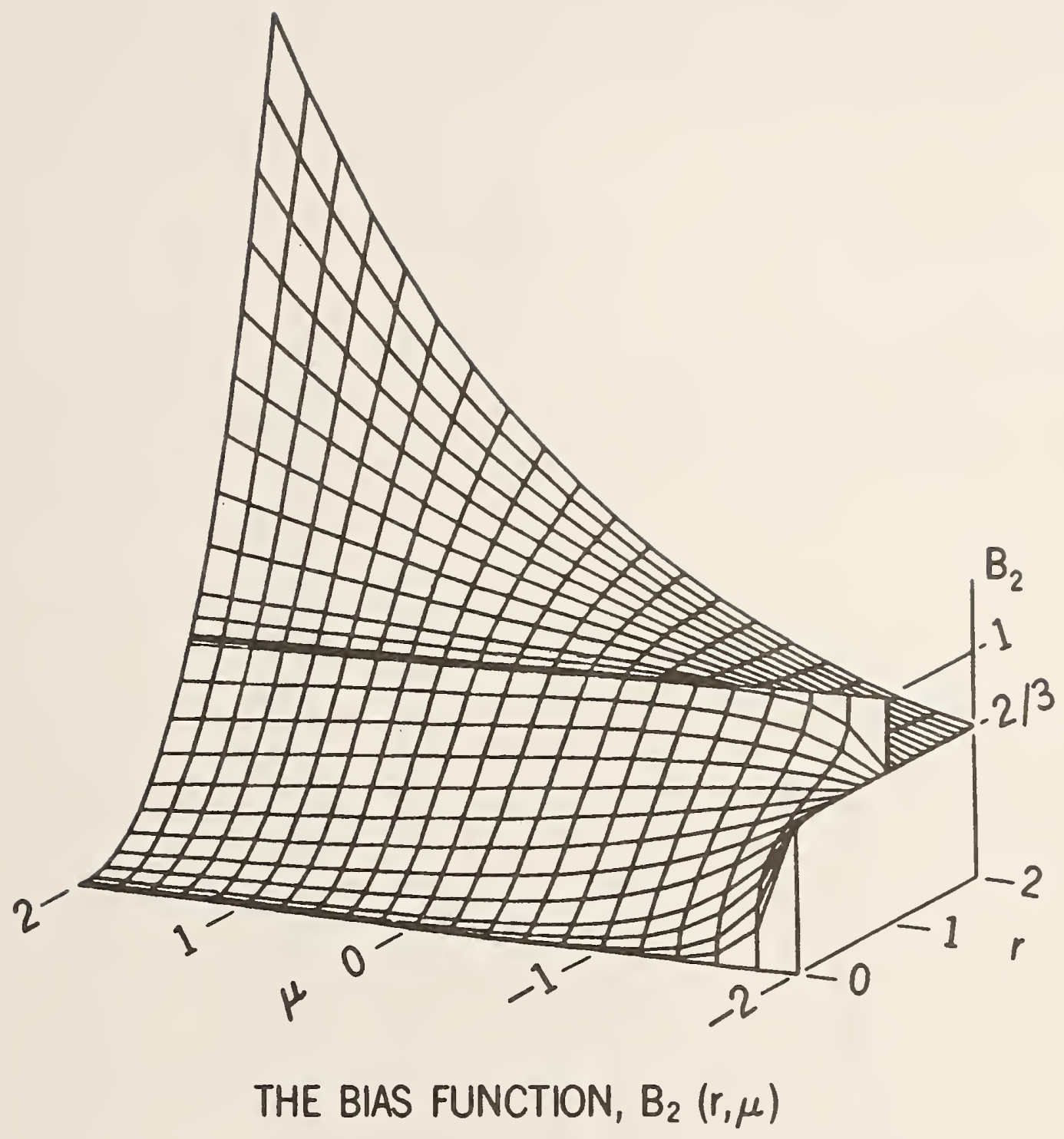

Figure 3. A three dimensional plot of the bias function $B_{2}(r, \mu)$, where $r=T / r$, and the dead time is $T-r$. The "fin" at $r=1$ and $\mu=-2$ approaches zero width as the measurement bandwidth approaches infinity (see appendix ref. [3]). 


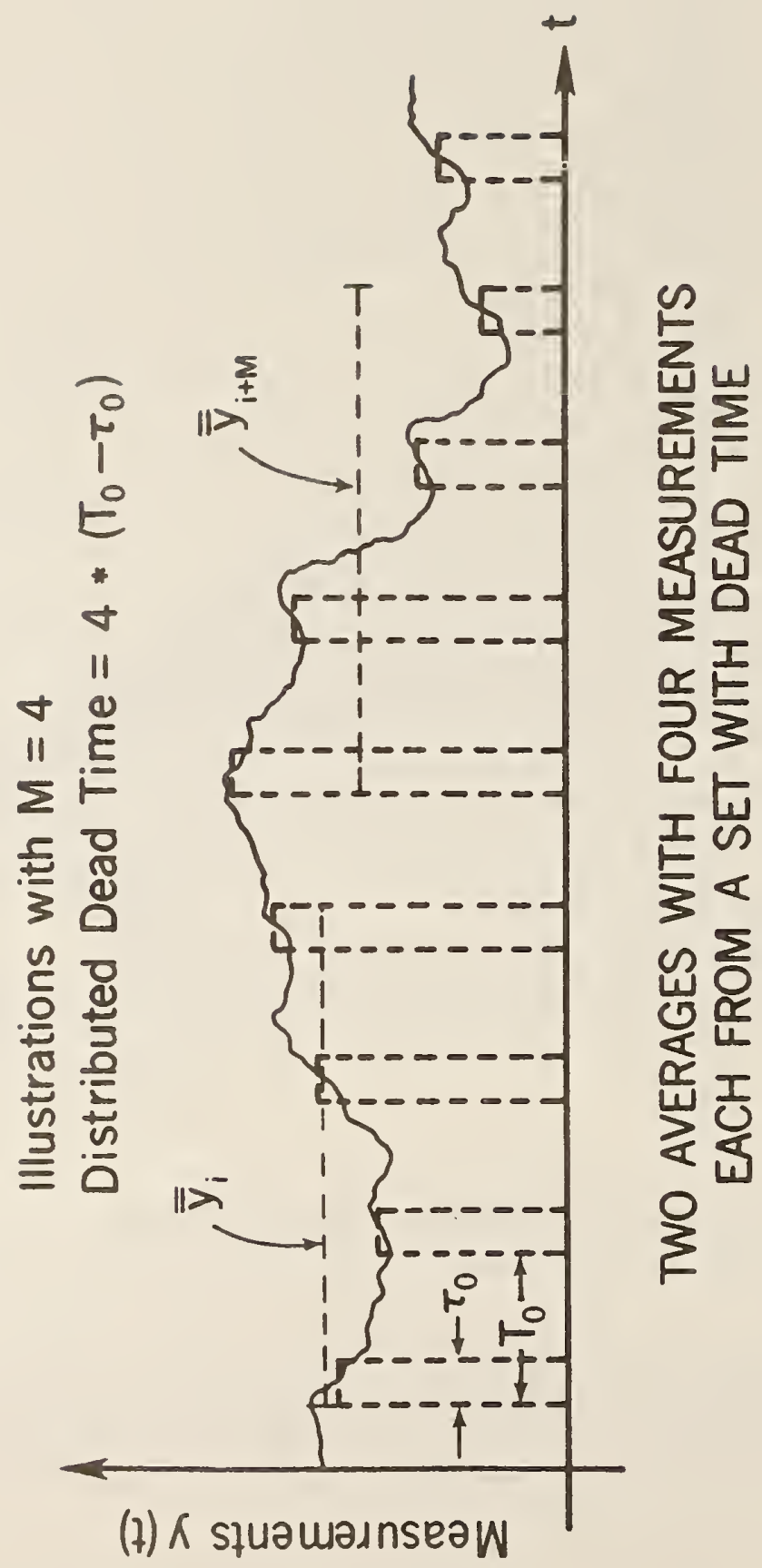

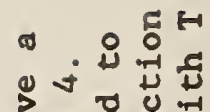

क II 过

되 에

n 乌ु요

- 1 ध

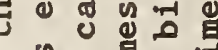

叫貝。

का मू

ه मी

H 1 क 4 (1)

iे 500

ह

월 웅

ए

है थ

政基

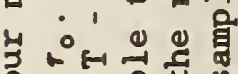

, 11

○ 每! 变

จำำ

自, 政

데 0 욤

tis

तु

a

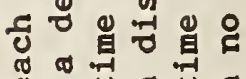

व व

ర

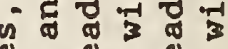

8 व

$\pi \circ 0$ \%

- 0 क

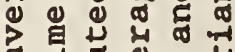

๙

०

$\rightarrow$ स 4

을 11

寻

年

๘ ๙

हैं is

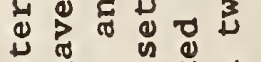

㟒

व $\rightarrow \infty$ क

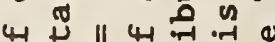

○

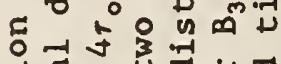

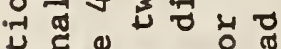

๘

म्र

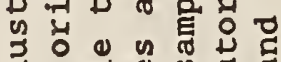

ב.

겅 을

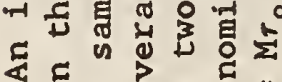
ธี

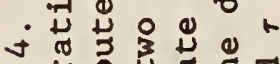

。出苟 3 उु कू

車 


\section{Appendix}

With reference to figure 1, the frequency sampling window has an equivalent phase sampling window. The intent is to evaluate the variance, $S(M)$, of the sampled phase function in terms of the phase autocorrelation function, $R(\tau)$. The process here is to correctly account for terms and cross-terms coming from squaring and averaging the samples for each $M$. The $B_{3}(2, M, r, \mu)$ function can then be obtained from the relation,

$$
B_{3}(2, M, r, \mu)=\frac{S(M)}{S(1) \cdot M^{\mu+2}}
$$

for appropriate $M, r$, and $\mu$. The denominator is just the two-sample variance with dead time for MT and Mr (in accordance with the definition of $\left.B_{3}(2, M, r, \mu)\right)$. The factors common to the numerator and denominator are ignored in the following.

For $M=1$, the variance $S(1)$ is just

$$
S(1)=4 \cdot R(0)-4 \cdot R(\tau)-4 \cdot R(T)+2 \cdot R(T+\tau)+2 R(T-\tau)=
$$

where use has been made of the definition of the autocorrelation function,

$$
R(T)=E[\phi(t) \cdot \phi(t+T)]
$$

It is convenient to define a function $G(T)$ as

$$
G(T)=2 \cdot R(T)-R(T+\tau)-R(T-\tau)
$$

Similarly, $S(2)$ can now be written in the form,

$$
S(2)=8 \cdot R(0)-8 \cdot R(\tau)+2 \cdot G(T)-4 \cdot G(2 T)-2 \cdot G(3 T) .
$$

Following this procedure, we can verify that the general $S(M)$ is just 


$$
\begin{aligned}
S(M) & =4 \cdot M \cdot R(0)-4 \cdot M \cdot R(\tau)-2 \cdot M \cdot G(M T) \\
& +2 \sum_{n=1}^{M-1}(M-n)[2 \cdot G(n T)-G((M+n) T)-G((M-n) T)] .
\end{aligned}
$$

Following the work of Barnes and Allan [2,3], we can define the function $U(\tau)$ by the relation,

$$
U(\tau)=2 \cdot R(0)-2 \cdot R(\tau)
$$

and also define

$$
F(n r)=G(n T) / U(r)
$$

where $r=T / \tau$. The function $U(\tau)$ for power-law power spectral densities has the form,

$$
U(\tau)=\frac{|\tau|^{\mu+2}}{4-2^{\mu+2}}
$$

which yields

$$
F(n r)=2 \cdot(n r)^{\mu+2}-(n r+1)^{\mu+2}-|n r-1|^{\mu+2}
$$

Finally, the working relation can be written as

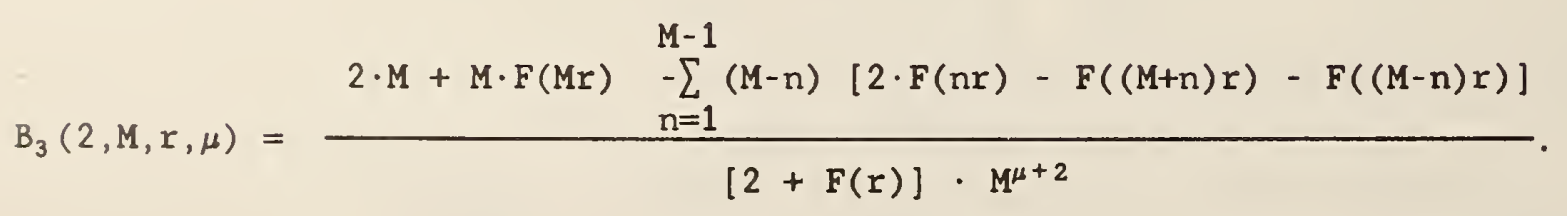


$B 1(N, r, \omega)$ for $r=.01$

\begin{tabular}{|c|c|c|c|c|c|c|c|c|c|c|c|}
\hline in 1 & $N=$ & 4 & 8 & 16 & 32 & 64 & 128 & 256 & 512 & 1024 & INF \\
\hline-2 & 8 & $1.000 E+\infty 0$ & $1.000 \varepsilon+00$ & 1. $000 E+\infty 0$ & $1.000 E+\infty 0$ & $1.000 E+\infty$ & $1.000 \varepsilon+00$ & $1.000 E+\infty$ & $1.000 E+\infty$ & 1. $000 E+\infty 0$ & $1.000 E+\infty$ \\
\hline-1.8 & 8 & $1.091 E+\infty 0$ & $1.210 E+\infty$ & $1.360 E+\infty 0$ & $1.545 E+00$ & $1.772 E+\infty$ & $2.00 \% E+\infty 0$ & $2.349 E+00$ & $2.456 E+\infty 0$ & $2.494 E+00$ & $2.51 x+\infty$ \\
\hline-1.6 & 1 & $1.199 E+\infty$ & $1.48 \pi+\infty 0$ & $1.8935+00$ & $2.455 E+00$ & $3.23 \% E+\infty 0$ & 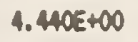 & $5.505 E+00$ & $6.002 E+00$ & $6.198 E+00$ & $6.30 \% E+\infty 0$ \\
\hline-1.4 & 8 & $1.328 E+\infty 0$ & $1.8565+\infty 0$ & $2.608 E+00$ & $3.991 E+\infty 0$ & $6.054 E+\infty 0$ & $9.500 \leq+00$ & $1.282 E+01$ & $1.456 E+01$ & $1.532 E+01$ & $1.565 E+01$ \\
\hline-1.2 & : & $1.482 \approx+00$ & $2.346 E+\infty 0$ & $3.800 E+\infty 0$ & $6.5902+00$ & $1.145 E+01$ & $2.026 E+01$ & $2.94 \pi+01$ & $3.486 E+01$ & 3. TSAE+01 & 3.990< +01 \\
\hline-1 & 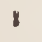 & $1.667 E+\infty 0$ & $3.000 E+00$ & $5.66 \pi+00$ & $1.100 E+01$ & $2.16 \pi+01$ & 4.250E+01 & $6.628 \varepsilon+01$ & $8.190 E+01$ & $9.064 E+01$ & 1.000E +02 \\
\hline-.8 & 8 & $1.884 E+00$ & $3.860 E+00$ & $8.303 E+\infty 0$ & $1.828 E+01$ & 4.041E+01 & $8.691 E+01$ & $1.443 E+02$ & $1.868 E+02$ & $2.137 E+02$ & $2.519 E+02$ \\
\hline-.6 & 1 & $2.134 E+\infty 0$ & $4.959 E+\infty 0$ & $1.2055+01$ & $2.97 \pi+01$ & $7.296 E+01$ & $1.69 \pi E+02$ & $2.995+02$ & $4.076 E+02$ & $4.851 E+02$ & $6.423 E+02$ \\
\hline-.4 & 8 & $2.407 E+\infty 0$ & $6.27 \%+00$ & $1.7035+01$ & $4.655 \mathrm{E}+01$ & $1.24 \pi+02$ & $3.106 E+02$ & $5.814 E+02$ & 8. $357 E+02$ & $1.043 E+03$ & $1.715 E+03$ \\
\hline-.2 & 8 & $2.677 E+\infty 0$ & $7.714 E+\infty 0$ & $2.2965+01$ & $6.8065+01$ & $1.976 E+02$ & 5. $220 E+02$ & $1.036 E+03$ & $1.500 E+03$ & $2.084 E \div 03$ & $5.581 E+03$ \\
\hline 0 & 1 & $2.91 \approx+00$ & $9.075 E+00$ & 2.909E+01 & $9.282 E+01$ & 2.857 & & & $9 E+03$ & $3.826 E+03$ & \\
\hline .2 & 8 & $3 . \cos x+\infty$ & $1.018 \varepsilon+01$ & $3.448+01$ & $1.16 x+02$ & $3.763 E+02$ & $1.097 E+03$ & $2.446 E+03$ & $4.263 E+03$ & $6.455 E+03$ & \\
\hline .4 & 8 & $3.203 E+\infty 0$ & $1.095 E+01$ & 3. $857 E+01$ & $1.354 E+02$ & $4.57 z+02$ & $1.390 E+03$ & $3.28 \pi E+03$ & $6.17 x+03$ & $1.014 E+04$ & \\
\hline .6 & : & $3.268<+\infty$ & $1.143 E+01$ & $4.1335+01$ & $1.495 E+02$ & $5.221 E+02$ & $1.649 E+03$ & $4.142 \varepsilon+03$ & $8.41 \%+03$ & $1.514 E+04$ & \\
\hline .8 & 8 & $3.302 E+\infty 0$ & $1.170 E+01$ & $4.303 E+01$ & $1.590<+02$ & 5. $708 E \div 02$ & $1.86 \%=03$ & $4.991 E+03$ & 1.104E+04 & $2.18 \% \varepsilon+04$ & \\
\hline 1 & 8 & $3.319 E+\infty 0$ & $1.185 E+01$ & $4.403 E+01$ & $1.653 E+02$ & $6.0665+02$ & $2.055 E+03$ & $5.842 E+03$ & $1.412 E+04$ & 3. $109 \varepsilon+04$ & \\
\hline 1.2 & $:$ & $3.32 \pi+00$ & $1.1925+01$ & $4.461 E+01$ & 1.693E +02 & 6. $300 E+02$ & $2.215 E+03$ & $6.717 E+03$ & $1.78 z \leq+04$ & $4.385 E+04$ & \\
\hline 1.4 & 8 & $3.350 E+\infty 0$ & $1.196 E+01$ & $4.494 E+01$ & $1.720 E+02$ & $6.530 E \div 02$ & $2.35 \%+03$ & $7.640 E+03$ & $2.234 E+04$ & $6.169 E+04$ & \\
\hline 1.6 & : & $3.332 E+\infty 0$ & $1.198 E+01$ & 4.514E+01 & $1.736 E+02$ & $6.680 E \div 02$ & $2.493 E+03$ & $8.637 E+03$ & $2.793 E+04$ & 8.697E+04 & \\
\hline 1.8 & $:$ & 3. $330^{2} E+\infty 0$ & $1.19 \% E+01$ & $4.526 E+01$ & $1.750 E \div 02$ & $6.819 E+02$ & $2.623 E+03$ & $9.73 \pi+03$ & $3.493 E+04$ & $1.231 E+05$ & \\
\hline 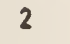 & : & $3.333 \varepsilon+00$ & $1.200 E+01$ & $4.533 E+01$ & $1.760 E+02$ & $6.933 E+02$ & $2.752 E+03$ & $1.097 E+04$ & $4.378 E+04$ & $1.749 E+05$ & \\
\hline
\end{tabular}

$B 1(N, r, m)$ for $r=.03$

\begin{tabular}{|c|c|c|c|c|c|c|c|c|c|c|c|}
\hline 41 & $N=$ & 4 & 8 & 16 & 32 & 64 & 128 & 256 & 512 & 1024 & INF \\
\hline-2 & 8 & $1.000 E+\infty 0$ & $1.000 E+\infty$ & $1.000 \varepsilon+\infty$ & $1.000 E+\infty$ & $1.000 E+00$ & $1.000 E+00$ & $1.000 E+00$ & $1.000 E+00$ & $1.000 E+\infty 0$ & 1. $000 E+\infty 0$ \\
\hline-1.8 & $:$ & $1.091 E+\infty$ & $1.211 E+\infty 0$ & $1.366 E+\infty 0$ & $1.573 E+\infty$ & $1.827 E+00$ & $1.950 E+00$ & $1.995 E+00$ & $2.010 E+00$ & $2.015 E+\infty$ & $E+\infty$ \\
\hline-1.6 & : & $1.1995+\infty 0$ & $1.491 E+\infty 0$ & $1.910 E+\infty 0$ & $2.532 E+\infty$ & $3.341 E+\infty 0$ & $3.782 E+\infty 0$ & $3.9635+00$ & $4.030 E+00$ & $4.053 E+\infty 0$ & $E+\infty$ \\
\hline-1.4 & $:$ & 1. $329 E+00$ & $1.863 E+00$ & $2.719 E * 00$ & $4.133 E+\infty 0$ & $6.000 E+00$ & $7.2685+00$ & $7.815 E+00$ & $8.044 E+00$ & $8.135 E+\infty 0$ & 8.19 \\
\hline-1.2 & : & $1.484 E+\infty 0$ & $2.355 E+00$ & $3.918 \varepsilon+\infty$ & $6.768 E+\infty 0$ & $1.093 E+01$ & $1.378 \varepsilon+01$ & $1.525 E+01$ & $1.5 \% 5 E+01$ & $1.626 E+01$ & $1.651 E+01$ \\
\hline-1 & $:$ & $1.667 E+\infty 0$ & 3. $000 E+\infty 0$ & $5.66 \pi t+\infty$ & $1.100 E+01$ & $1.928 E+01$ & $2.560 E+01$ & $2.929 E+01$ & 3.127E+01 & 3.22\%+01 & 3. $3335+01$ \\
\hline-.8 & $:$ & $1.878 \varepsilon+00$ & $3.825 E+00$ & $8.141 E+\infty 0$ & 1. $7535+01$ & $E+01$ & $4.626 E+01$ & $5.49 \% \Leftarrow+01$ & $6.029 E+01$ & $6.341 E+01$ & $6.770 E+01$ \\
\hline. .6 & : & $2.114 E+00$ & $4.860 E+00$ & $1.147 E+01$ & $2.706 E+01$ & $5.41 E+01$ & $8.04 \% E+01$ & $9.997 E+01$ & $1.134 E+02$ & $1.225 E+02$ & $1.403 E+02$ \\
\hline .4 & $:$ & $2.362 E+100$ & $6.005 E+00$ & $1.566 E+01$ & $3.993 E+01$ & $8.524 E+01$ & $1.335 E+02$ & $1.744 E+02$ & $2.066 E+02$ & $2.314 E+02$ & $3.099 E+02$ \\
\hline-.2 & : & $2.604 E+\infty 0$ & $7.242 E+00$ & $2.047 E+01$ & $5.501 E+01$ & $1.25 \%+02$ & $2.094 E+02$ & $2.897 E+02$ & $3.61 \%+02$ & $4.256 E+02$ & 8.5 \\
\hline 0 & : & $2.819 E+\infty 0$ & $8.4305+00$ & $2.546 E+01$ & $7.3485+01$ & $1.749 E+02$ & $3.096 E+02$ & $4.568 E+02$ & $6.08 \not E+02$ & $7.611 E+02$ & \\
\hline .2 & : & $2.992 E+00$ & $9.460 E+00$ & $3.013 E+01$ & $9.127 E+01$ & $2.286 E+02$ & $4.324 E+02$ & $6.856 E+02$ & $9.834 E+02$ & $1.328 E+03$ & \\
\hline .4 & $:$ & $3.118 E+\infty 0$ & $1.027 E+01$ & 3. $412 E+01$ & $1.077 E+02$ & $2.835 E+02$ & 5. $750 E+02$ & $9.868 E+02$ & $1.541 E+03$ & $2.2 T \pi E+03$ & \\
\hline .6 & $:$ & $3.203 E+00$ & $1.087 E+01$ & $3.728 \varepsilon+01$ & $1.220 E+02$ & $E+02$ & $7.357 E+02$ & $1.376 E+03$ & $2.364 E+03$ & 3. $868 E+03$ & \\
\hline .8 & : & 3. $257 E+\infty 0$ & $1.128 E+01$ & $3.966 E+01$ & $1.339 E+02$ & 3. $887 E+02$ & $9.152 E+02$ & $1.87 \%+03$ & $3.500 E+03$ & $6.554 E+03$ & \\
\hline 1 & $:$ & $3.290 E+00$ & $1.155 E+01$ & $4.141 E+01$ & $1.437 E+02$ & 4. $382 E+02$ & $1.117 E \div 03$ & $2.533 E+03$ & $5.395 E+03$ & $1.114 E+04$ & \\
\hline 1.2 & : & 3. $309+00$ & $1.172 E+01$ & 4.267E+01 & $1.518 E+02$ & $4.867 E+02$ & $1.348 E+03$ & $3.392 x+03$ & $8.128 E+03$ & $1.9035+04$ & \\
\hline 1.4 & $:$ & $3.320 E+\infty 0$ & $1.184 E+01$ & 4. $360 E+01$ & $1.5885 * 02$ & $5.35 z+02$ & $1.615 E+03$ & 4.535E $\div 03$ & $1.228 \varepsilon+04$ & $3.276 E+04$ & \\
\hline 1.6 & : & $3.327 E+\infty 0$ & $1.191 E+01$ & $4.431 E+01$ & $1.650 E+02$ & $5.851 E+02$ & $1.930 E+03$ & $6.068 \varepsilon+03$ & $1.866 E+04$ & $5.684 E+04$ & \\
\hline 1.8 & $:$ & 3. $331 E+00$ & $1.196 E+01$ & 4. 487E+01 & $1.706 E+02$ & $6.374 E+02$ & $2.304 E+03$ & $8.142 E+03$ & $2.849 E+04$ & $9.936 E+04$ & \\
\hline 2 & : & 3. $333 E+\infty$ & $1.200 E+01$ & $4.533 E+01$ & $1.760 E+02$ & $6.933 E+02$ & $2.75 x+03$ & $1.097 E+04$ & $4.378 \varepsilon+04$ & $1.749 \varepsilon+05$ & \\
\hline
\end{tabular}


$B 1(N, r, w)$ for $r * .1$

\begin{tabular}{|c|c|c|c|c|c|c|c|c|c|c|c|}
\hline N 1 & $N$ & 4 & 8 & 16 & 32 & 64 & 128 & 256 & 512 & 1024 & INF \\
\hline-2 & : & $1.000 E+\infty 0$ & $1.000 E+\infty$ & 1.000E+D & $1.000 E+\infty 0$ & $1.000 E+\infty$ & $1.000 E+\infty 0$ & $1.000 E+00$ & $1.000 E+\infty 0$ & 1.000E++0 & $0 E+00$ \\
\hline-1.8 & : & $1.093 E+\infty 0$ & $1.226 E+\infty$ & $1.438 E+\infty 0$ & $1.54 \pi+\infty$ & 1.579E+00 & $1.586 E+\infty 0$ & $1.506 E+\infty 0$ & $1.585 E+00$ & $1.584 E+\infty 0$ & $1.582 E+\infty 0$ \\
\hline-1.6 & : & $1.205 E+\infty 0$ & $1.522 E+00$ & $2.021 E+\infty 0$ & $2.329 E+\infty 0$ & $2.44 \%+\infty$ & $2.490 E+00$ & $2.502 E+\infty 00$ & $2.500 \bar{t}+00$ & $2.50 \sigma t+00$ & $2.504 E+00$ \\
\hline-1.4 & : & 1.337E++0 & 1. $90 \pi+\infty$ & $2.832 \varepsilon+\infty 0$ & 3. $47 \pi E+\infty$ & $3.7725+\infty$ & $3.892 E+\infty 0$ & $3.938 E+00$ & $3.9 \% 1 E+\infty 0$ & $3.960 E+\infty 0$ & $3.962 \leq+\infty 0$ \\
\hline-1.2 & : & $1.491 E+\infty$ & $2.396 E+00$ & $3.931 E+\infty 0$ & $5.125 E+\infty 00$ & $5.751 E+00$ & $6.0465+00$ & $6.177 E+\infty$ & $6.235 E+\infty 0$ & $6.260 E+00$ & $6.278 E+\infty 0$ \\
\hline-1 & $:$ & $1.66 \pi E+\infty 0$ & $3.000 E+\infty 0$ & $5.375 E+\infty 0$ & $7.42 \%+\infty+\infty$ & $8.653 E+\infty$ & $9.31 z+00$ & $9.652 E+\infty 00$ & $9.82 x E+\infty 0$ & $9.91 x E+\infty$ & 1.000E+01 \\
\hline-.8 & : & $1.860 \varepsilon+\infty 0$ & $3.720 E+\infty 0$ & $7.204 E+\infty 0$ & $1.055 E+01$ & $1.281 E+01$ & $1.41 \%+01$ & $1.502 E+01$ & $1.550 E+01$ & $1.57 \pi+01$ & $1.616 E+01$ \\
\hline-.6 & : & $2.065 E+\infty 00$ & $4.540 E+\infty 0$ & $9.423 E+\infty 0$ & $1.461 E+01$ & $1.8595+01$ & $2.13 \pi+01$ & $2.326 E+01$ & 2. $452 E+01$ & $2.53 \pi+01$ & $2.70 x E+01$ \\
\hline-.4 & : & $2.273 E+\infty 0$ & $5.430 E+\infty 0$ & $1.19 \%+01$ & $1.971 E+01$ & $2.642 E+01$ & 3.177E+01 & $3.5925+01$ & $3.910 E+01$ & $4.152 E+01$ & $4.92 x E+01$ \\
\hline-.2 & : & $2.472 E+00$ & $6.344 E+\infty 0$ & $1.481 E+01$ & $2.565 E+01$ & $3.6735+01$ & $4.664 E+01$ & $5.5425+01$ & $6.314 E+01$ & $6.9995+01$ & $E+02$ \\
\hline 0 & : & $2.653 E+\infty 0$ & $7.236 E+00$ & 1. $77 \% E+01$ & 3. $300 E+01$ & 5. $003 E+01$ & $6.771 E+01$ & $8.566 E+01$ & $1.03 \pi+02$ & $1.21 \%+02$ & \\
\hline .2 & : & $2.810 E+00$ & $8.065 E+\infty 0$ & 2. $080 E+01$ & 4.111E+01 & $6.690 E+01$ & $9.750 E+01$ & $1.331 E+02$ & $1.741 E+02$ & $2.214 E+02$ & \\
\hline .4 & : & $2.940 E+\infty 0$ & $8.804 E+\infty 0$ & 2.376E+01 & $5.015 E+01$ & $8.816 E+01$ & $1.397 E+02$ & $2.0845+02$ & $2.993 E+02$ & $4.194 E+02$ & \\
\hline .6 & : & $3.043 E+\infty 0$ & $9.445 E+\infty 0$ & $2.663 E+01$ & $6.014 E+01$ & $1.1495+02$ & $1.9995+02$ & $3.295 E+02$ & $5.265 E+02$ & 8. $254 E+02$ & \\
\hline .8 & : & $3.123 E+\infty 0$ & $9.989 E+00$ & $2.938 E+01$ & $7.118 \varepsilon+01$ & $1.48 \pi+02$ & $2.862 E+02$ & $5.26 \% \Leftarrow+02$ & $9.466 \varepsilon+02$ & $1.678 E+03$ & \\
\hline 1 & $:$ & $3.184 E+00$ & 1. $045 E+01$ & $3.204 E+01$ & 8. $347 E+01$ & $1.918 E+02$ & $4.114 E+02$ & 8. $\overline{2} 235+02$ & $1.735 E+03$ & $3.500 E+03$ & \\
\hline 1.2 & : & $3.230 E+\infty 0$ & $1.084 E+01$ & 3. $463 E+01$ & $9.726 E+01$ & $2.470 E+02$ & $5.943 E+02$ & $1.394 E+03$ & 3. $232 x^{\circ}+03$ & $7.4535+03$ & \\
\hline 1.4 & : & $3.265 E+00$ & $1.118 E+01$ & $3.721 E+01$ & 1. $130 E+02$ & $3.183 E+02$ & $8.636 E+02$ & $2.304 E+03$ & $6.103 E+03$ & $1.613 E+04$ & \\
\hline 1.6 & : & $3.293 E+00$ & $1.147 E+01$ & $3.982 E+01$ & 1. $309 E+02$ & 4. $113 E+02$ & $1.263 E+03$ & $3.8432+03$ & $1.166 E+04$ & $3.535 E+04$ & \\
\hline 1.8 & : & $3.315 E+00$ & $1.174 E+01$ & 4. $252 E+01$ & $1.517 E+02$ & $5.330 \varepsilon+02$ & $1.858 E+03$ & $6.467 E+03$ & $2.250 E+04$ & $7.830 E+04$ & \\
\hline 2 & $\cdot:$ & 3. $333 E+00$ & $1.200 E+01$ & $4.5335+01$ & $1.760 E+02$ & $6.933 E+02$ & $2.752 \varepsilon+03$ & $1.097 E+04$ & $4.378 E+04$ & $1.74 \%+05$ & \\
\hline
\end{tabular}

$B 1(N, r, m)$ for $r=.3$

\begin{tabular}{|c|c|c|c|c|c|c|c|c|c|c|c|}
\hline Mu 1 & $N=$ & 4 & 8 & 16 & 32 & 64 & 128 & 256 & 512 & 1024 & INF \\
\hline-2 & : & $1.000 E+\infty$ & $1.000 E+\infty$ & $1.000 E+\infty 0$ & $00 E+\infty$ & 1. $000 E+\infty 0$ & $1.000 E+00$ & $1.000 E+\infty 0$ & $1.000 E+00$ & $1.000 E+00$ & $=\$ 00$ \\
\hline-1.8 & : & $1.124 E+\infty 0$ & $1.226 E+00$ & $1.259 E+\infty 0$ & $1.265 E+00$ & $1.265 E+00$ & $1.263 E+00$ & $1.262 E+\infty$ & $1.261 E+00$ & $1.261 E+\infty 0$ & $1.260 E+00$ \\
\hline-1.6 & : & 1. $254 E+\infty$ & $1.479 E+\infty 0$ & $1.564 E+00$ & $1.589 E+\infty 0$ & $1.594 E+00$ & $1.594 E+00$ & $1.593 E+\infty 0$ & $1.591 E+C 0$ & $1.591 E+00$ & $1.590 E+00$ \\
\hline-1.4 & : & $1.388 E+\infty 0$ & $1.75 \% E+\infty 0$ & $1.925 E+00$ & $1.9888+00$ & $2.009 E+00$ & $2.014 E+\infty 0$ & $2.015 E+\infty 0$ & $2.015 E+\infty 0$ & $2.014 E+\infty 0$ & $2.013 E+00$ \\
\hline-1.2 & : & $1.527 E+\infty 0$ & $2.068 E+00$ & $2.351 E+00$ & $2.479 E+00$ & $2.534 E+00$ & $2.556 E+\infty 0$ & $2.565 E+\infty 0$ & $2.569 E+\infty 0$ & $2.570 E+00$ & $2.571 E+\infty$ \\
\hline-1 & : & $1.667 E+0.0$ & $2.405 E+\infty 0$ & $2.850 \varepsilon+100$ & $3.087 E+00$ & $3.209 E+00$ & 3. $271 E+\infty$ & $3.302 E+\infty 0$ & $3.318 E+00$ & $3.326 E+\infty 0$ & $3.333 E+\infty 0$ \\
\hline-.8 & : & 1. $806 E+\infty 0$ & $2.76 \%+\infty 0$ & $3.435 E+00$ & $3.847 E+\infty 0$ & $4.093 E+00$ & $4.23 \%+\infty 0$ & $4.325 E+\infty 0$ & $4.376 E+00$ & $4.405 E+00$ & $4.447 \varepsilon+00$ \\
\hline. .6 & : & $1.943 E+00$ & $3.160 E+00$ & $4.122 E+00$ & $4.804 E+00$ & $5.275 E+00$ & $5.595 E+\infty 0$ & $5.812 E+\infty 0$ & $5.958 E+00$ & $6.056 E+00$ & 6. $252 E+00$ \\
\hline-.4 & : & $2.076 E+O D$ & $3.577 E+00$ & $4.927 E+\infty 0$ & $6.024 E+\infty 0$ & $6.889 E+\infty 0$ & $7.564 E+\infty 0$ & $8.086 E+00$ & $8.48 \pi+00$ & $8.795 E+00$ & $9.776 E+\infty 0$ \\
\hline-.2 & : & $2.203 E+\infty 0$ & $4.021 E+00$ & $5.877 E+00$ & $7.597 E+\infty 0$ & $9.147 E+00$ & $1.053 E+01$ & $1.175 E+01$ & $1.282 E+01$ & 1. $377 \mathrm{E}+01$ & $2.017 E+01$ \\
\hline 0 & : & $2.325 E+00$ & $4.492 E+00$ & $7.000 E+100$ & $9.651 E+\infty 0$ & $1.2385+01$ & $1.515 E+01$ & $1.796 E+01$ & $2.079 E+01$ & $2.363 E+01$ & \\
\hline .2 & : & $2.440 E+00$ & $4.994 E+00$ & 8. $338 E+00$ & $1.236 E+01$ & $1.710 E+01$ & $2.260 E+01$ & $2.897 E+01$ & $3.632 E+01$ & $4.479 E+01$ & \\
\hline .4 & : & $2.55 C E+\infty 0$ & $5.530 E+00$ & $9.940 E+00$ & $1.599 E+01$ & $2.411 E+01$ & 3.493E+01 & $4.926 E+01$ & $6.823 E+01$ & $9.330 E+01$ & \\
\hline .6 & : & $2.654 E+00$ & $6.106 E+00$ & $1.187 E+0 !$ & $2.089 E+01$ & $3.472 E+01$ & $5.581 E+01$ & $8.786 E+01$ & $1.365 E+02$ & $2.103 E+02$ & \\
\hline .8 & : & $2.754 E+100$ & $6.729 E+\infty 0$ & $1.422 E+0 !$ & $2.756 E+01$ & $5.098 E+01$ & $9.187 E+01$ & $1.632 E+02$ & $2.874 E+02$ & $5.037 E+02$ & \\
\hline 1 & : & $2.852 E+\infty 0$ & $7.406 E+\infty 00$ & 1. $708 E+01$ & $3.673 E+01$ & $7.618 E+01$ & $1.552 E+02$ & $3.132 E+02$ & $6.292 E+02$ & $1.261 E+03$ & \\
\hline 1.2 & : & $2.947 E+00$ & 8. $147 E+00$ & $2.05 \%+01$ & $4.942 E+01$ & $1.157 E+02$ & $2.678 E+02$ & $6.170 E+02$ & $1.419 E+03$ & $3.261 E+03$ & \\
\hline 1.4 & : & $3.042 x+00$ & $8.963 E+100$ & $2.493 E+01$ & $6.709 E+01$ & $1.781 E+02$ & $4.703 E+02$ & $1.241 E+03$ & 3. $272 E+03$ & 8. $631 E+03$ & \\
\hline 1.6 & : & $3.137 E+00$ & $9.868 E+00$ & 3.030E+01 & $9.185 E+01$ & 2. 774E+02 & 8. $381 E+02$ & $2.535 E+03$ & $7.673 E+03$ & $2.324 E+04$ & \\
\hline 1.8 & : & $3.234 E+00$ & $1.087 E+01$ & $3.699 E+01$ & $1.267 E+02$ & $4.366 E+02$ & $1.511 E+03$ & 5. $245 E+03$ & $1.823 E+04$ & $6.343 E+04$ & \\
\hline 2 & : & 3. $323 E+00$ & $1.200 E+01$ & $4.533 E+01$ & $1.760 E+02$ & $6.933 E+02$ & $2.752 E+03$ & $1.097 E+04$ & 4. $378 E+04$ & $1.749 E+05$ & \\
\hline
\end{tabular}


$B 1(N, r, w)$ for $r=1$

\begin{tabular}{|c|c|c|c|c|c|c|c|c|c|c|c|}
\hline Hu 1 & $\mathbb{N}=$ & 4 & 8 & 16 & 32 & 64 & 128 & 256 & 512 & 1024 & INF \\
\hline-2 & : & 8.33కE-01 & $7.500 E-01$ & $7.083 E-01$ & $6.875 E-01$ & $6.771 E-01$ & $6.719 E-01$ & $6.693 E-01$ & $6.600 E-01$ & $6.673 E-01$ & $6.667 \mathrm{E}-01$ \\
\hline-1.8 & : & $8.581 E-01$ & $7.827 E-01$ & $7.431 E-01$ & $7.226 E-01$ & $7.122 E-01$ & $7.0685-01$ & $7.042 E-01$ & $7.028 E-01$ & $7.021 E-01$ & $7.014 E-01$ \\
\hline-1.6 & : & $8.866 E-01$ & $8.221 E-01$ & $7.864 E-01$ & $7.6725-01$ & $7.570 E-01$ & $7.51 \pi-01$ & $7.490 E-01$ & $7.476 E-01$ & $7.468 E-01$ & $7.461 E-01$ \\
\hline-1.4 & : & $9.193 E-01$ & $8.700 E-01$ & $8.410 E-01$ & $8.245 x-01$ & 8.154E-01 & 8.105E-01 & $8.079 E-01$ & 8.065E-01 & 8. $058 E-01$ & $8.051 E-01$ \\
\hline-1.2 & : & $9.56 \% E-01$ & $9.284 E-01$ & $9.105-01$ & $8.997 E-01$ & $8.933 E-01$ & $8.89 \pi-01$ & 8.877E-01 & 8.866E-01 & $8.860 E-01$ & $8.854 E-01$ \\
\hline-1 & $:$ & $1.000 E+\infty 0$ & $1.000 E+\infty 0$ & $1.000 E+\infty 0$ & $1.000 E+\infty 0$ & $1.000 E+00$ & $1.000 E+\infty$ & $1.000 E+\infty 0$ & $1.000 E+\infty 0$ & $1.000 E+00$ & $1.000 E+00$ \\
\hline-.8 & $:$ & $1.050 E+\infty$ & $1.068 E+00$ & $1.11 \pi+\infty$ & $1.137 E+\infty 00$ & $1.150 E+\infty$ & $1.160 E+\infty$ & $1.165 E+00$ & $1.169 E+00$ & $1.171 E+00$ & $1.175 E+00$ \\
\hline-.6 & : & $1.10 \pi+\infty$ & $1.19 \pi+\infty 0$ & $1.271 E+\infty$ & $1.327 E+\infty$ & $1.370 E+\infty 0$ & $1.401 E+\infty$ & $1.422 E+\infty$ & $1.438 x+00$ & 1. $448 E+00$ & $1.470 E+00$ \\
\hline-.4 & : & $1.172 E+\infty$ & $1.333 E+\infty$ & $1.476 E+\infty 0$ & $1.59 \%+\infty$ & $1.700 E+\infty$ & $1.782 \geq+\infty$ & $1.847 E+\infty 0$ & $1.898 E+\infty 00$ & $1.938 E+\infty 0$ & $2.065 E+00$ \\
\hline-.2 & : & $1.247 E+\infty$ & $1.502 \pm+\infty 0$ & $1.754 E+\infty 0$ & $1.994 E+\infty 0$ & $2.216 E+\infty$ & $2.418 \varepsilon+\infty$ & $2.59 \%+00$ & $2.75 \%+\infty$ & 2. $900 E+\infty 0$ & $3.863 E+00$ \\
\hline 0 & : & $1.333 E+00$ & $1.714 E+\infty 0$ & $2.133 E+\infty 0$ & $2.581 E+\infty 0$ & $3.048 E+\infty 0$ & $3.528 \varepsilon+\infty 0$ & $4.016 E+\infty 0$ & 4.50\%E+00 & $5.005 E+00$ & \\
\hline .2 & : & $1.432 E+00$ & $1.982 E+\infty$ & $2.658 E+\infty$ & $3.471 E+\infty$ & $4.432 E+\infty$ & $5.555 E+\infty$ & $6.858 E+\infty 0$ & $8.363 E+\infty 0$ & $1.010 E+01$ & \\
\hline .4 & : & $1.546 E+00$ & $2.320 E+\infty 0$ & $3.391 E+\infty$ & $4.846 E+\infty 0$ & $6.801 E+\infty$ & $9.40 \pi+\infty$ & 1. $28 \pi E+01$ & $1.744 E+01$ & 2. $350 E+01$ & \\
\hline .6 & : & $1.677 E+\infty$ & $2.750 E+\infty$ & $4.424 E+\infty 0$ & $7.006 E+00$ & $1.096 E+01$ & $1.698 E+01$ & $2.614 E+01$ & 4.005E+01 & $6.114 E+01$ & \\
\hline .8 & : & $1.827 E+\infty$ & $3.299 E+\infty 0$ & $5.894 E+\infty$ & $1.045 E+01$ & $1.841 E+01$ & $3.230 E+01$ & $5.6525+01$ & $9.872 \Sigma+01$ & $1.722 E+02$ & \\
\hline 1 & : & $2.000 E+00$ & 4. $000 E+00$ & 8. $000 E+00$ & $1.600 E+01$ & $3.200 E+01$ & $6.400 E+01$ & $1.280 E+02$ & $2.560 E+02$ & $5.120 E+02$ & \\
\hline 1.2 & : & $2.198 E+\infty 0$ & 4. $900 E+\infty 0$ & $1.104 E+01$ & $2.506 E+01$ & $5.71 \pi+01$ & $1.308 E+02$ & $2.999 E+02$ & $6.881 E+02$ & $1.580 E+03$ & \\
\hline 1.4 & : & $2.426 E+\infty 0$ & $6.059 E+\infty 0$ & $1.5465+01$ & $3.999 E+01$ & $1.04 E+02$ & $2.738 E+02$ & $7.202 \varepsilon+02$ & $1.89 \pi+03$ & $5.003 E+03$ & \\
\hline 1.6 & : & $2.688 E+00$ & $7.555 E+\infty$ & $2.191 E+01$ & $6.479 E+01$ & $1.938 E+02$ & $5.833 E+02$ & $1.762 \varepsilon+03$ & $5.331 E \div 03$ & $1.615 E+04$ & \\
\hline 1.8 & : & $2.988 E+\infty 0$ & $9.490 E+\infty 0$ & $3.1385+01$ & $1.063 E+02$ & $3.646 E+02$ & $1.260 E+03$ & $4.372 E+03$ & $1.519 E+04$ & $5.286 E+04$ & \\
\hline 2 & : & $3.333 E+\infty 0$ & $1.200 E+01$ & $4.533 E+01$ & $1.760 E+02$ & $6.933 E+02$ & $2.75 X E+03$ & $1.097 E+04$ & $4.378 E+04$ & $1.749 E+05$ & \\
\hline
\end{tabular}

$B 1(N, r, m)$ for $r=1.01$

\begin{tabular}{|c|c|c|c|c|c|c|c|c|c|c|c|}
\hline w 1 & $N=$ & 4 & 8 & 16 & 32 & 64 & 128 & 256 & 512 & 1024 & INF \\
\hline-2 & : & $1.000 E+\infty$ & $1.000 E+\infty 0$ & $1.000 E+\infty 0$ & $1.000 E+\infty 0$ & $1.000 E+\infty$ & $1.000 E+00$ & $1.000 E+00$ & $1.000 E+00$ & $1.000 E+\infty 0$ & 1.000E+00 \\
\hline-1.8 & : & $9.156 E-01$ & $8.682 E-01$ & $8.425 E-01$ & $8.2885-01$ & $8.217 E-01$ & $8.181 E-01$ & $8.162 E-01$ & $8.153 E-01$ & $8.148 E-01$ & $8.143 E-01$ \\
\hline-1.6 & : & $9.098 E-01$ & $8.566 E-01$ & $8.264 E-01$ & $8.098 \varepsilon-01$ & $8.009 E-01$ & $7.963 E-01$ & $7.936 \mathrm{E}-01$ & $7.926 E-01$ & $7.920 E-01$ & $7.913 E-01$ \\
\hline-1.4 & : & $9.286 E-01$ & $8.840 E-01$ & $8.573 E-01$ & $8.41 \% E-01$ & 8. 333E-01 & $8.287 \mathrm{E}-01$ & $8.262 E-01$ & $8.249 E-01$ & $8.242 E-01$ & $8.235 E-01$ \\
\hline-1.2 & : & $9.5995-01$ & $9.331 E-01$ & $9.160 E-01$ & $9.056 E-01$ & $8.995 E-01$ & $8.960 E-01$ & $8.940 E-01$ & $8.929 E-01$ & $8.924 E-01$ & $8.917 E-01$ \\
\hline-1 & : & $1.000 E+\infty 0$ & $1.000 E+00$ & $1.000 E+\infty 0$ & $1.000 E+\infty$ & $1.000 E+00$ & 1. $000 E+00$ & $1.000 E+\infty 0$ & $1.000 E+00$ & $1.000 E+\infty 0$ & $1.000 E+\infty 0$ \\
\hline-.8 & $:$ & $1.048 E+\infty 0$ & $1.085 E+00$ & $1.113 E+\infty$ & $1.133 E+\infty$ & $1.147 E+\infty$ & $1.155 E+100$ & $1.161 E+\infty$ & $1.165 E+\infty 0$ & $1.167 E+\infty$ & $1.170 E+\infty 00$ \\
\hline-.6 & : & $1.104 E+\infty 0$ & $1.193 E+\infty 0$ & $1.265 E+\infty$ & $1.321 E+\infty$ & $1.36 \boldsymbol{x}+00$ & $1.393 E+\infty 0$ & $1.414 E+\infty 0$ & $1.42 \%+\infty$ & 1. $440 E+00$ & $1.461 E+\infty$ \\
\hline-.4 & : & $1.168 E+\infty$ & $1.327 E+\infty 0$ & $1.468 E+\infty$ & $1.589 E+\infty 0$ & $1.68 \% E+\infty 0$ & $1.770 E+\infty$ & $1.835 E+\infty 0$ & $1.885 E+\infty 0$ & $1.924 E+00$ & $2.050 E+\infty 0$ \\
\hline-.2 & : & $1.243 E+00$ & $x+\infty 0$ & $1.743 E+\infty$ & $1.980=+\infty$ & 2.200 & $2.400 E+\infty 0$ & $2.579 E+\infty$ & $2.737 E+\infty$ & $2.877 E+\infty 0$ & $+\infty$ \\
\hline 0 & : & 1. $32 \% E+\infty$ & $1.706 E+\infty 0$ & $2.120 E+\infty$ & $2.5635+00$ & $3.025 E+\infty 0$ & $3.500 E+\infty 0$ & $3.984 E+\infty 00$ & $4.472 \leq+\infty$ & $4.963 E+00$ & \\
\hline .2 & $:$ & $1.428 E+00$ & $1.9725+\infty 0$ & $2.642 E+\infty 0$ & $3.44 \pi+\infty$ & 4. $400 E+00$ & $5.512 E+\infty$ & $6.804 E+\infty 0$ & $8.296 E+\infty$ & $1.002 \varepsilon+01$ & \\
\hline .4 & : & $1.541 E+\infty 0$ & $2.309 E+\infty 0$ & $3.371 E+\infty 0$ & $4.814 E+\infty 0$ & $6.754 E+\infty 0$ & $9.340 E+00$ & $1.27 \pi+01$ & $1.731 E+01$ & $2.332 E+01$ & \\
\hline .6 & : & $1.672 x+\infty 0$ & $2.739 \varepsilon+\infty 0$ & $4.400 E+\infty 0$ & $6.9635+00$ & $1.08 \% E+01$ & $1.68 \pi E+01$ & $2.59 \pi+01$ & $3.978 \varepsilon+01$ & $6.073 E+01$ & \\
\hline .8 & $:$ & $1.822 E+00$ & $3.205 E+\infty 0$ & $5.864 E+00$ & $1.0395+01$ & 1.830 & $3.212 E+01$ & $5.619 E+01$ & $9.814 E+01$ & $1.712 \varepsilon+02$ & \\
\hline 1 & $:$ & $1.995 E+\infty 0$ & $3.985 E+\infty 0$ & $7.966 E+\infty 0$ & $1.5935 \div 01$ & 3.185E+01 & $6.369 E+01$ & $1.274 E+02$ & 2.547E+02 & $5.095 E+02$ & \\
\hline 1.2 & : & $2.194 E+\infty 0$ & $4.8005 E+\infty$ & $1.100 E+01$ & $2.49 \pi E+01$ & $5.69 x+01$ & $1.303 E+02$ & $2.987 E+02$ & $6.854 E+02$ & $1.573 E+03$ & \\
\hline 1.4 & $:$ & $2.422 E+\infty 0$ & $6.045 E+\infty$ & $1.5425+01$ & 3. $900 E+01$ & $1.041 E+02$ & $2.730 E+02$ & $7.180 E+02$ & $1.892 \mathbf{E}+03$ & $4.988 E+03$ & \\
\hline 1.6 & : & $2.685 E+\infty 0$ & $7.542 \pi+00$ & $2.18 \pi E+01$ & $6.466 E+01$ & $1.934 E+02$ & $5.822 x+02$ & $1.758 E+03$ & $5.321 E+03$ & $1.611 E+04$ & \\
\hline 1.8 & $:$ & $2.986 E+\infty 0$ & $9.482 x+\infty$ & $3.135 E+01$ & $1.062 E+02$ & $3.643 E+02$ & $1.25 \% \Leftarrow+03$ & $4.367 E+03$ & $1.518 E+04$ & 5. $200 E+04$ & \\
\hline 2 & $:$ & 3. $335 E+\infty 0$ & 1. $200 E+01$ & $4.5335+01$ & $1.760 E+02$ & $6.933 E+02$ & 2. $752 \mathbb{E}+03$ & $1.097 E+04$ & $4.378 E+04$ & $1.749 E+05$ & \\
\hline
\end{tabular}


$B I(N, r, m u)$ for $r=1.1$

\begin{tabular}{|c|c|c|c|c|c|c|c|c|c|c|c|}
\hline Hos 1 & $N=$ & 4 & 8 & 16 & 32 & 64 & 128 & 256 & 512 & 1024 & INF \\
\hline-2 & : & $1.000 E+\infty 0$ & $1.000 E+00$ & $000 E+\infty$ & $1.000 E+00$ & $1.000 E+00$ & $1.000 E+\infty 0$ & $C O O E+\infty O$ & $1.000 E+00$ & $1.000 E+\infty 0$ & $1.000 E+\infty 0$ \\
\hline-1.8 & : & $9.527 E-01$ & $9.243 E-01$ & $9.082 E-01$ & $8.994 E-01$ & $8.948 E-01$ & $8.924 E-01$ & $3.911 E-01$ & 8. $905 E-01$ & $8.902 E-01$ & $8.898 E-01$ \\
\hline-1.6 & $:$ & $9.415 E-01$ & $9.048 E-01$ & $8.831 E-01$ & $8.70 \%-01$ & $8.642 E-01$ & $8.607 E-01$ & $8.588 E-01$ & $8.578 E-01$ & $8.574 E-01$ & $8.569 E-01$ \\
\hline-1.4 & $:$ & $9.493 E-01$ & $9.1575-01$ & $8.948 E-01$ & $8.825 E-01$ & $8.755 E-01$ & $8.717 E-01$ & $8.696 E-01$ & $8.685 E-01$ & $8.680 E-01$ & $8.674 E-01$ \\
\hline-1.2 & : & $9.696 E-01$ & $9.482 E-01$ & $9.341 E-01$ & $9.254 E-01$ & $9.201 E-01$ & $9.171 E-01$ & $9.154 E-01$ & $9.145 E-01$ & $9.140 E-01$ & $9.134 E-01$ \\
\hline-1 & : & $1.000 E+00$ & $1.000 E+\infty 0$ & $1.000 E+\infty 0$ & $1.000 E+00$ & $1.000 E+00$ & 1. $000 E+00$ & $1.000 E+00$ & $1.000 E+\infty$ & $1.000 E+00$ & 1. $000 E+00$ \\
\hline-.8 & : & 1. $040 E+00$ & $1.072 E+\infty$ & $1.096 E+00$ & $1.114 E+00$ & 1.126 & $1.134 E+\infty 0$ & $1.13 \%+00$ & $1.14 \mathbb{x}+\infty$ & $1.144 E+\infty 0$ & $1.147 E+\infty 0$ \\
\hline-.6 & : & $1.088 E+00$ & $1.166 E+00$ & $1.230 E+00$ & $1.281 E+00$ & $1.319 E+\infty 0$ & $1.346 E+\infty 0$ & $1.366 E+\infty 0$ & $1.37 \% E+00$ & $1.38 \%+00$ & $1.408 E+00$ \\
\hline-.4 & : & $1.147 E+00$ & $1.288 E+\infty 0$ & $1.416 E+\infty$ & $1.526 E+00$ & $1.617 E+\infty 0$ & $1.692 \pm+00$ & $1.751 E+\infty$ & $1.797 E+\infty 0$ & $1.83 x \leq+\infty$ & $1.948 E+00$ \\
\hline-.2 & : & $1.217 E+00$ & $1.444 E+\infty 0$ & $1.671 E+\infty 0$ & $1.889 E+\infty 0$ & 2.091 & $2.275 E+\infty$ & $2.440 E \div 00$ & $2.586 E+\infty 0$ & $2.714 E+\infty 0$ & $3.593 E+00$ \\
\hline 0 & $:$ & $1.298 E+00$ & $1.643 E+00$ & $2.026 E+\infty 0$ & $2.435 E+00$ & $2.863 E+00$ & $3.304 E+00$ & $3.752 E+00$ & $4.205 E+\infty 0$ & $4.661 E+\infty 0$ & \\
\hline .2 & : & 1. $393 E+\infty 0$ & $1.8985+00$ & $2.521 E+\infty 0$ & $3.272 E+\infty$ & $4.160 E+00$ & $5.199 E+00$ & $6.404 E+\infty 0$ & $7.797 E+00$ & $9.402 E+\infty 0$ & \\
\hline .4 & : & $1.504 E+00$ & $2.223 E+00$ & $3.219 E+00$ & $4.575 E+\infty 0$ & $6.398 E+00$ & $8.828 E+00$ & $1.205 E+01$ & $1.632 E+01$ & $2.197 E+01$ & \\
\hline .6 & $:$ & $1.633 E+\infty 0$ & $2.640 E+00$ & $4.213 E+\infty 0$ & $6.63 \% E+00$ & $1.035 E+01$ & $1.602 E+01$ & 2. $463 E+01$ & 3. $771 E+01$ & $5.754 E+01$ & \\
\hline .8 & $:$ & $1.7835+00$ & $3.177 E+\infty 0$ & $5.637 E+\infty 0$ & $9.954 E+\infty 0$ & 1. $750 E+01$ & $3.068 E+01$ & $5.365 E+01$ & $9.3665+01$ & $1.634 E+02$ & \\
\hline 1 & : & $1.957 E+\infty 0$ & $3.870 E+00$ & $7.696 E+00$ & $1.535 \mathrm{E}+01$ & $3.065 E+01$ & $6.126 E+01$ & $1.225 E+02$ & $2.449 E+02$ & $4.898 E+02$ & \\
\hline 1.2 & : & $2.158 \varepsilon+\infty$ & $4.766 E+00$ & $1.069 E+01$ & $2.422 E+01$ & $5.521 E+01$ & $1.263 E+02$ & $2.894 E+02$ & $6.640 E+02$ & $1.524 E+03$ & \\
\hline 1.4 & $:$ & $2.391 E+100$ & $5.929 E+100$ & $1.508 E+01$ & 3.897E+01 & $1.016 E+02$ & $2.666 E+02$ & $7.011 E+02$ & 1.847E+03 & $4.870 E+103$ & \\
\hline 1.6 & $:$ & $2.6600+00$ & $7.443 E+\infty 0$ & $2.154 E+01$ & $6.366 E+01$ & $1.904 E+02$ & $5.730 E+02$ & $1.731 E+03$ & 5.237E+03 & $1.586 E+04$ & \\
\hline 1.8 & : & $2.97 Z E+00$ & $9.418 E+00$ & $3.111 E+01$ & $1.053 E+02$ & $3.614 E+02$ & $1.249 E+03$ & 4. $3335+03$ & $1.506 E+04$ & $5.238 E+04$ & \\
\hline 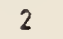 & : & $3.333 E+100$ & $1.200 E+01$ & $4.533 E+01$ & $1.760 E+02$ & $6.933 E+02$ & $2.752 E+03$ & $1.097 E+04$ & $4.378 E+04$ & $1.749 E+05$ & \\
\hline
\end{tabular}

$B !(N, r, m u)$ for $r=2$

\begin{tabular}{|c|c|c|c|c|c|c|c|c|c|c|c|}
\hline Hos 1 & $N=$ & 4 & 8 & 16 & 32 & 64 & 128 & 256 & 512 & 1024 & INF \\
\hline-2 & $:$ & 1. $000 E+\infty 0$ & $1.000 E+00$ & $1.000 E+00$ & $1.000 E+\infty$ & $1.000 E+00$ & 1. $000 E+\infty 0$ & $1.000 E+00$ & $1.000 E+00$ & $1.000 E+\infty$ & 1.000Et \\
\hline-1.8 & $:$ & $9.901 E-01$ & $9.836 E-01$ & $9.796 E-01$ & $9.774 E-01$ & $9.761 E-01$ & $9.755 E-01$ & $9.752 E-01$ & $9.750 E-01$ & $9.749 E-01$ & $9.748 E-01$ \\
\hline-1.6 & $:$ & $9.845 E-01$ & $9.737 E-01$ & $9.669 E-01$ & $9.630 E-01$ & $9.607 E-01$ & $9.595 E-01$ & $9.589 E-01$ & $9.586 E-01$ & $9.584 E-01$ & $9.58 z E-01$ \\
\hline-1.4 & $:$ & $9.837 E-01$ & $9.719-01$ & $9.641 E-01$ & S.593E-01 & $9.565 E-01$ & $9.550 E-01$ & $9.541 E-01$ & $9.537 E ̈-01$ & $9.534 E-01$ & $9.532 E-01$ \\
\hline-1.2 & $:$ & $9.886 E-01$ & $9.799 E-01$ & $9.738 E-01$ & $9.699 E-01$ & $9.675 E-01$ & $9.661 E-01$ & $9.653 E-01$ & $9.648 E-01$ & $9.646 E-01$ & $9.643 E-01$ \\
\hline-1 & $:$ & $1.000 E+\infty 0$ & $1.000 E+100$ & $1.000 E+\infty 0$ & $1.000 E+00$ & $1.000 E+\infty 0$ & $1.000 E+\infty$ & $1.000 E+00$ & $1.000 E+00$ & $1.0005+\infty$ & $1.000 E+00$ \\
\hline-.8 & $:$ & $1.019 E+00$ & $1.035 E+00$ & $1.048 E+00$ & $1.058 E+\infty$ & $1.064 E+00$ & $1.069 E+\infty 0$ & $1.072 E+\infty 0$ & $1.074 E+\infty 0$ & $1.075 E+\infty$ & $1.077 E+\infty 0$ \\
\hline-.6 & $:$ & $1.046 E+00$ & $1.090 E+00$ & $1.126 E+00$ & $1.156 E+00$ & $1.178 E+\infty 0$ & $1.195 E+\infty$ & $1.207 E+\infty$ & $1.215 E+\infty 0$ & $1.221 E+00$ & $1.2335+\infty$ \\
\hline$-.4^{\circ}$ & $:$ & $1.084 E+00$ & $1.168 E+00$ & $1.246 E+\infty 0$ & $1.315 E+\infty 0$ & $1.373 E+00$ & $1.420 E+\infty 0$ & $1.457 E+\infty 0$ & $1.487 E+\infty 0$ & $1.509 E+\infty 0$ & $1.583 E+00$ \\
\hline-.2 & $:$ & $1.133 E+\infty 0$ & $1.277 E+00$ & $1.425 E+00$ & $1.568 \varepsilon+00$ & $1.702 E+00$ & $1.824 E+\infty$ & $1.934 E+00$ & $2.031 E+00$ & $2.117 E+00$ & $2.704 E+00$ \\
\hline 0 & : & $1.195 E+00$ & $1.427 E+00$ & $1.689 E+00$ & $1.971 E+\infty 0$ & $2.267 E+00$ & $2.573 \xi+\infty$ & $2.884 E+00$ & $3.198 E+\infty 0$ & $3.515 E+\infty 0$ & \\
\hline .2 & $:$ & $1.273 E+\infty 0$ & $1.62 \% E+00$ & $2.075 E+00$ & $2.615 E+\infty$ & $3.256 E+00$ & $4.005 E+\infty$ & $4.876 E+\infty 0$ & $5.882 E+00$ & $7.042 E+\infty$ & \\
\hline .4 & $:$ & 1. $36 \% E+00$ & $1.901 E+00$ & $2.644 E+00$ & $3.65 \%+\infty$ & $5.025 E+00$ & $6.847 E+00$ & $9.267 E+00$ & $1.247 E+01$ & $1.670 E+01$ & \\
\hline .6 & $:$ & $1.486 E+00$ & $2.264 E+00$ & $3.485 E+00$ & $5.371 E+\infty$ & $8.262 E+00$ & $1.267 E+01$ & $1.937 E+01$ & $2.955 E+01$ & $4.498 E+01$ & \\
\hline .8 & $:$ & $1.628 E+00$ & $2.750 E+00$ & $4.733 E+\infty 0$ & $8.215 E+00$ & $1.431 E+01$ & $2.494 E+01$ & $4.34 \pi E+01$ & $7.576 E+01$ & $1.320 E+02$ & \\
\hline 1 & $:$ & $1.800 E+00$ & $3.400 E+00$ & $6.600 E+00$ & $O E+01$ & $2.580 \varepsilon+01$ & OE+01 & +02 & $2.050 E+02$ & $4.098 E+02$ & \\
\hline 1.2 & $:$ & $2.007 E+00$ & $4.271 E+\infty 0$ & $9.40 \% E+00$ & $2.114 E+01$ & $4.800 E+01$ & $1.096 E+02$ & $2.510 E+02$ & 5. $756 E+02$ & $1: 321 E+03$ & \\
\hline 1.4 & $:$ & $2.255 E+00$ & $5.439 E+00$ & $1.366 E+01$ & $3.512 E+01$ & $9.142 E+01$ & $2.395 E+02$ & $6.299 E+02$ & $1.659 E+03$ & $4.374 E+03$ & \\
\hline 1.6 & : & $2.552 E+\infty 0$ & $7.012 E+\infty 0$ & $2.014 E+01$ & $5.935 E+01$ & $1.773 E+02$ & $5.334 E+02$ & $1.611 E+03$ & $4.874 E+03$ & $1.476 E+04$ & \\
\hline 1.8 & $:$ & $2.900 E+00$ & $9.132 E+00$ & $3.006 E+01$ & $1.017 E+02$ & 3. $487 E+02$ & $1.205 E+03$ & $4.17 \%+03$ & $1.453 E+04$ & $5.053 E+04$ & \\
\hline 2 & : & $3.333 E+\infty 0$ & $1.200 E+01$ & $4.533 E+01$ & $1.760 E+02$ & $6.933 E+02$ & $2.752 E+03$ & 1.097E+04 & $4.378 E+04$ & $1.74 \%+05$ & \\
\hline
\end{tabular}


B1 $(W, r, w)$ for $r=4$

\begin{tabular}{|c|c|c|c|c|c|c|c|c|c|c|c|}
\hline 4 & $*$ & 4 & 8 & 16 & 32 & 64 & 128 & 206 & 512 & 1024 & IfF \\
\hline 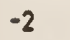 & 8 & $1.000 E+\infty 0$ & $1.000 E+00$ & $1.000 E+00$ & 1. $000 E+\infty 0$ & $1.000=00$ & $1 \mathrm{ADAC}+\infty$ & 1.000E+CO & 1. $000 E+\infty 0$ & $1.000 E+\infty$ & 1. $000 E+\infty$ \\
\hline 1.8 & 8 & $9.974 E-01$ & $9.95 \pi-01$ & $9.944 E-01$ & $9.940 E-01$ & $9.936 E-01$ & $9.7735 E-01$ & $2.934 E-01$ & $9.935 E-01$ & $9.936 E-01$ & $9.9355-01$ \\
\hline-1.6 & 8 & $9.952 x-01$ & $9.918 E-01$ & $9.896 \varepsilon-01$ & $9.884 E-01$ & $9.876 E-01$ & $9.073 E-01$ & $9,870 E-01$ & $9.86 \%-01$ & $9.86 \%-01$ & $9.8686-01$ \\
\hline-1.4 & 8 & $9.941 E-01$ & $9.6965-01$ & $9.869=01$ & $9.851 E-01$ & $9.840 E-01$ & $9.88=01$ & $7.801 E-01$ & $9.829 E-01$ & $9.829 E-01$ & $9.82 \%<-01$ \\
\hline-1.2 & $!$ & $9.95 x E-01$ & $9.916 E-01$ & $9.890<-01$ & $9.873 E-01$ & $9.86 z-01$ & $9.656 E-01$ & $9.855 E-01$ & $9.851 E-01$ & $9.850 E-01$ & $9.849 E-01$ \\
\hline-1 & 8 & $1.000 E+\infty 0$ & 1. $000 E+\infty$ & $1.000 E+\infty 0$ & 1. $000 E+\infty$ & $1.000 E+\infty$ & 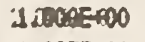 & 1.000E+00 & $1.000 E+\infty$ & 1. $000 E+\infty$ & $1.000 E+\infty$ \\
\hline .8 & : & $1.010<+\infty 0$ & $1.019+\infty$ & $1.026 E+\infty 0$ & $1.031 E+\infty$ & $1.035 E+\infty 0$ & 1.03 & 1.039 & $1.040 E+\infty$ & $1.041 E+\infty 0$ & $1.04 z+\infty$ \\
\hline-.6 & 8 & $1.027 E+\infty 0$ & $1.053 E+00$ & $1.075 E+\infty 0$ & $1.093 E+00$ & $1.107 E+00$ & 1.117 & $1.124 E+\infty$ & $1.12 \%+\infty$ & $1.132 E+\infty 0$ & 1. $140 E+\infty$ \\
\hline-.4 & 8 & $1.054 E+\infty 0$ & $1.10 \%+00$ & $1.160 E+\infty 0$ & $1.205 E+\infty 0$ & 1.2 & 1.2 & 12 & $1.318 E+\infty$ & $1.335 E+\infty$ & $1.38 x+\infty$ \\
\hline .2 & 1 & $1.092 E+\infty 0$ & $1.194 E+00$ & $1.29 \% E+\infty 0$ & $1.39 \%+\infty$ & 1.49 & 1. $.500 E+\infty 0$ & 1865 & $1.727 E+\infty$ & $1.780 E+\infty$ & $2.204 E+00$ \\
\hline 0 & 1 & $1.144 E+\infty 0$ & $1.318 E+\infty 0$ & $1.514 E+\infty$ & $1.726 E+\infty 0$ & $1.949=100$ & $2: 5795+\infty 0$ & 2.414 & $2.651 E+\infty$ & $2.890 E+\infty 0$ & \\
\hline .2 & 8 & $1.214 E+\infty$ & $1.495 E+\infty 0$ & $1.848 E+\infty 0$ & $2.275 E+00$ & $2.780 E+C D$ & .3. 3TRE+60 & .4 .067 & $4.865 E+\infty 0$ & $5.784 E+\infty 0$ & \\
\hline .4 & 8 & $1.303 E+\infty 0$ & $1.742 x+00$ & $2.3565+\infty 0$ & $3.196 E+\infty 0$ & $4.326 E+100$ & $: 8.8$ & 7.8 & $1.049 E+01$ & $1.399 E+01$ & \\
\hline .6 & 8 & $1: 415 E+00$ & $2.0825+00$ & $3.12 \%++\infty 0$ & $4.748 E+\infty 0$ & $7.22 s E+\infty 0$ & & & 2. $550 E+01$ & $3.875 E+01$ & \\
\hline .8 & 8 & $1.555 E+\infty$ & $2.548 E+00$ & 4. $303 E+\infty 0$ & $7.305+\infty$ & $1.278 E+01$ & $2.21 \%+01$ & $3.886 E+01$ & $6.718 E+01$ & $1.170 E+02$ & \\
\hline 1 & 8 & $1.727 E+\infty 0$ & $3.18 z+00$ & $6.091 E+\infty 0$ & $1.191 E+01$ & $2.355 E+01$ & $40085+01$ & $9.3365+01$ & $1.865 E+02$ & $3.726 E+02$ & \\
\hline 1.2 & $:$ & $1.938 E+00$ & $4.045 E+00$ & $8.826 E \div 00$ & $1.974 E+01$ & $4.472 E+01$ & $1.026 \mathrm{EFO2}$ & 2.336E+02 & $5.356 E+02$ & $1.229 E+03$ & \\
\hline 1.4 & 8 & $2.194 E+\infty 0$ & $5.219 E+\infty$ & $1.303 E+01$ & $3.340 E+01$ & $8.686 E+a 1$ & $2 \pi \pi \leq+02$ & 5.t281Et02 & $1.575 E+03$ & $4.154 E+03$ & \\
\hline 1.6 & $:$ & $2.504 E+00$ & $6.819 E+00$ & $1.95 x+01$ & $5.745 E+01$ & & & & $4.714 E+03$ & $1.428 E+04$ & \\
\hline 1.8 & : & $2.87 \%++\infty$ & $9.006 E+00$ & $2.960 E+01$ & 1. $000 E+02$ & $3.431 E+02$ & 1. $196 E+03$ & $4.1126+03$ & $1.42 \% E+04$ & $4.972 E+04$ & \\
\hline . & $:$ & $3.333 E+\infty 0$ & $1.200 E+01$ & $4.335 E+01$ & $1.760 E+02$ & $6.933 E+.02$ & $2.752 E+03$ & I'SPREA & $4.378 E+04$ & $1.749 E+05$ & \\
\hline
\end{tabular}

B1 $(N, r, w)$ for $r=8$

\begin{tabular}{|c|c|c|c|c|c|c|c|c|c|c|c|}
\hline 41 & $N=$ & 4 & 8 & 16 & 32 & 64 & 1588 & 256 & 512 & 1024 & INF \\
\hline 2 & : & $1.000 E+\infty 0$ & $1.000 E+\infty$ & $1.000 E+\infty 0$ & $1.000 E+\infty$ & 1.000E+teo & 11. $000 E+\infty$ & D. $600 E+00$ & $1.000 E+\infty$ & $1.000 E+00$ & 1.000E+O \\
\hline 1.8 & $:$ & $9.9935-01$ & $9.988 E-01$ & $9.985 E-01$ & $9.983 E-01$ & $9.982 E-01$ & $9.981 E-01$ & 9.98 & $9.981 E-01$ & $9.981 E-01$ & $9.981 E-01$ \\
\hline 1.6 & : & $9.984 E-01$ & $9.973 E-01$ & $9.966 E-01$ & $9.962 E-01$ & $9.960 E-01$ & $9.958 E-01$ & $9.758 E-01$ & $9.957 E-01$ & $9.957 E-01$ & $9.957 E-01$ \\
\hline 1.4 & $:$ & $9.978 E-01$ & $9.961 E-01$ & $9.950 E-01$ & $9.944 E-01$ & $9.940 E-01$ & 2.237E-01 & 2. $.336 \leq-01$ & $9.936 E-01$ & $9.935 E-01$ & $9.935 E-0.1$ \\
\hline 1.2 & : & $9.980 E-01$ & $9.963 E-01$ & $9.952 E-01$ & $9.945 E-01$ & $9.940 E-01$ & $9.738 E-01$ & $9.936 \mathrm{E}-01$ & $9.935 x-01$ & $9.935 E-01$ & $9.934 E-01$ \\
\hline 1 & : & $1.000 E+\infty$ & $1.000 E+\infty 00$ & $1.000 E+\infty 0$ & $1.000 E+\infty$ & 1. $000 E+\infty 0$ & $1.000 E+\infty 0$ & $1,000 E+00$ & $1.000 E+\infty 0$ & $1.000 E+00$ & $1.000 E+\infty 0$ \\
\hline .8 & : & $1.006 E+\infty 0$ & $1.011 E+\infty 0$ & $1.014 E+\infty 0$ & $1.017 E+\infty$ & $1.020 E+00$ & $: 1,021 E+\infty$ & & $1.022 E+\infty 00$ & $1.023 E+00$ & $1.023 E+\infty 0$ \\
\hline .6 & $:$ & $1.017 E+00$ & $1.033 E+\infty$ & $1.047 E+00$ & $1.058 E+\infty 0$ & $1.067 E+00$ & $11.073 E+00$ & :1:076E+00 & $1.081 E+\infty$ & $1.083 E+00$ & $1.088 E+\infty 0$ \\
\hline-.4 & 1 & $1.037 E+\infty 0$ & $1.075 E+\infty$ & $1.110 E+\infty 0$ & $1.142 E+\infty 0$ & & $1.18 \%++\infty$ & & $1.220 E+\infty 0$ & $1.231 E+\infty 0$ & $1.264 E+\infty 0$ \\
\hline-.2 & : & $1.06 \%+00$ & $1.145 E+\infty$ & $1.223 E+\infty$ & $1.29 \%+00$ & $1.371 E+\infty$ & 1. $436 E+\infty$ & 1.494 & $1.546 E+00$ & $1.592 E+\infty 0$ & $1.905 E+00$ \\
\hline 0 & : & $1.116 E+00$ & $1.255 E+\infty 0$ & $1.413 E+\infty 0$ & $1.584 E+\infty 0$ & $1.763 E+\infty 0$ & $1.949 E+\infty 0$ & 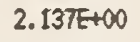 & $2.328 E+\infty$ & $2.520 E+\infty 0$ & \\
\hline .2 & : & $1.181 E+\infty 0$ & $1.420 E+00$ & $1.720 E+\infty$ & $2.083 E+\infty 0$ & $2.514 E+00$ & $3.019 E+\infty$ & $3.606 E+\infty$ & $4.284 E+00$ & 5. $066 E+00$ & \\
\hline .4 & : & $1.268 E+\infty 0$ & $1.657 E+\infty$ & $2.202 E+\infty 0$ & $2.946 E+\infty 00$ & $3.949 E+00$ & $-5.286 E+\infty 0$ & 7.062 & $9.414 E+\infty$ & $1.252 E+01$ & \\
\hline .6 & : & $1.380 E+\infty 0$ & $1.991 E+\infty 0$ & $2.950 E+00$ & $4.433 E+00$ & $6.706 E+00$ & $1.017 E+01$ & 1.544E+01 & $2.345 E+01$ & $3.55 \%+01$ & \\
\hline .8 & : & $1.521 E+\infty 0$ & $2.453 E+\infty 0$ & $4.101 E+\infty 0$ & $6.996 E+\infty 00$ & $1.206 E+01$ & $2.090 E+01$ & $3,630 E+01$ & $6.315 E+01$ & $1.099 E+02$ & \\
\hline 1 & : & $1.696 E+00$ & $3.087 E+\infty 0$ & $5.870 E+\infty 0$ & $1.143 E+01$ & $2.257 E+01$ & $4.483 E+01$ & $8.935 E+01$ & $1.784 E+02$ & $3.565 E+02$ & \\
\hline 1.2 & : & $1.910 E+\infty$ & $3.953 E+\infty 0$ & $8.5 \% 0 E+\infty$ & $1.91 \pi+01$ & $4.341 E+01$ & $9.989 E+01$ & $2.26 x+02$ & $5.195 E+02$ & $1.192 E+03$ & \\
\hline 1.4 & $:$ & $2.170 E+\infty 0$ & $5.135 E+\infty$ & $1.279 E+01$ & $3.276 E+01$ & $8.515 E+0.1$ & $2.230 E+02$ & $5.86 z+02$ & $1.544 E+03$ & $4.071 E+03$ & \\
\hline 1.6 & $:$ & $2.486 E+00$ & $6.751 E+\infty$ & $1.930 E+01$ & $5.678 E+01$ & $1.695 E+02$ & $5.099 E+02$ & $1.540 E+03$ & $4.658 E+03$ & $1.411 E+04$ & \\
\hline 1.8 & : & $2.870 E+00$ & $8.963 E+\infty 0$ & $2.945 E+01$ & $9.951 E+01$ & $3.413 E+02$ & 1. $1792+03$ & $4.0965+.03$ & $1.421 E+04$ & $4.945 E+04$ & \\
\hline 2 & : & 3. $333 E+00$ & $1.200 E+01$ & $4.533 E+01$ & $1.760 E+02$ & $6.933 E+02$ & $2.7520+03$ & $1.097 E+04$ & $4.378 E+04$ & $1.749 E+05$ & \\
\hline
\end{tabular}


$B 1(N, r, \ldots)$ for $r=16$

\begin{tabular}{|c|c|c|c|c|c|c|c|c|c|c|c|}
\hline 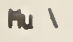 & $N=$ & 4 & 8 & 16 & 32 & 64 & 128 & 256 & 512 & 1024 & INF \\
\hline-2 & $:$ & $1.000 E+00$ & 1.000E++0 & $1.000 E+\infty 0$ & $1.000 E+\infty$ & $1.000 E+00$ & $1.000 E+00$ & $1.000 E+\infty 0$ & $1.000 E+\infty$ & $1.000 E+\infty 0$ & $1.000 E+00$ \\
\hline-1.8 & : & $9.9995-01$ & $9.997 E-01$ & $9.996 E-01$ & $9.9955-01$ & $9.9955-01$ & $9.9955-01$ & 9.995E-01 & $9.995 E-01$ & $9.9955-01$ & $9.9955-01$ \\
\hline-1.6 & 8 & $9.995-01$ & $9.991 E-01$ & $9.9895=01$ & $9.987 E-01$ & $9.987 E-01$ & 9.986E-01 & $9.9265-01$ & $9.9265-01$ & $9.9065-01$ & $9.9865-01$ \\
\hline-1.4 & $:$ & $9.992 z=01$ & $9.995 \times-01$ & $9.981 E-01$ & $9.97 \%=01$ & $9.97 \pi \mathrm{E}-01$ & $9.976 E-01$ & $9.976 E-01$ & $9.976 E-01$ & $9.975 E-01$ & $9.975 E-01$ \\
\hline-1.2 & $:$ & $9.991 E-01$ & $9.984 E-01$ & $9.97 \%=01$ & $9.976 E-01$ & $9.974 E-01$ & $9.9735=01$ & $9.972 \leq-01$ & $9.9725=01$ & $9.972<-01$ & $9.971 E-01$ \\
\hline-1 & $:$ & $1.0005+00$ & $1.000 E+\infty 0$ & $1.0005+00$ & $1.000 \leqslant+00$ & $1.000 E+\infty 0$ & $1.000 E+\infty 0$ & $1.000 E+00$ & $1.000 E+00$ & $1.000 E+00$ & $1.000 E+\infty$ \\
\hline-.8 & : & $1.0035+00$ & $1.006 E+\infty 0$ & $1.000 E+\infty 0$ & $1.010 E+00$ & $1.011 E+\infty 0$ & $1.012 \varepsilon+\infty$ & $1.0125+\infty$ & $1.013 \varepsilon+\infty 0$ & $1.013 E+\infty 0$ & $1.013 \Sigma+\infty$ \\
\hline. .6 & 8 & $1.011 E+\infty$ & $1.021 E+\infty 0$ & $1.030 E+\infty 0$ & $1.037 E+00$ & $1.0435+00$ & $1.047 E+\infty 0$ & $1.050 E+\infty$ & $1.052 \varepsilon+\infty 0$ & $1.0535+00$ & $1.0565+00$ \\
\hline-.4 & 8 & $1.026 E+00$ & $1.0535+\infty$ & $1.078 E+00$ & $1.101 E+00$ & $1.11 \%+\infty$ & $1.135 E+\infty$ & $1.14 \pi+\infty 0$ & $1.157 \mathrm{E}+00$ & $1.164 E+\infty 0$ & $1.188 E+\infty 0$ \\
\hline-.2 & : & $1.054 E+00$ & $1.113 E+00$ & $1.174 E+00$ & $1.233 E+\infty 0$ & $1.289 E+\infty 0$ & $1.30 \%+\infty$ & $1.336 E+00$ & $1.4252 \div 00$ & $1.461 E+\infty 0$ & $1.70 s E+00$ \\
\hline 0 & $:$ & $1.097 E+00$ & $1.214 E+\infty 0$ & $1.346 E+00$ & $1.48 \%+\infty 0$ & $1.63 \%+\infty 00$ & $1.794 E+\infty 0$ & $1.9525+\infty 0$ & $2.11 x+\infty 0$ & $2.273 E+\infty$ & \\
\hline .2 & : & $1.160 E+00$ & $1.37 \approx+00$ & $1.637 E+\infty 0$ & $1.958 E+\infty 0$ & $2.340 E+00$ & $2.787 E+\infty 0$ & $3.307 E+00$ & $3.90 \pi+\infty 0$ & 4.599E+00 & \\
\hline .4 & $:$ & $1.247 E+00$ & $1.606 E+\infty 0$ & $2.100 \Sigma+\infty 0$ & $2.794 E+00$ & $3.717 E++0$ & $4.950 E+00$ & $6.587 E+00$ & 8.75HE++0 & $1.162 x+01$ & \\
\hline .6 & : & $1.360 E+00$ & $1.9395+00$ & $2.8485+00$ & $4.251 E+00$ & 6. $409 \varepsilon+\infty 0$ & $9.696 E+00$ & $1.46 \%+01$ & $2.2285+01$ & $3.379 \varepsilon+01$ & \\
\hline .8 & : & $1.504 E+00$ & $2.404 E+00$ & $3.997 E+00$ & $6.794 \varepsilon+00$ & $1.169 E+01$ & $2.025 E+01$ & $3.5125+01$ & $6.1065+01$ & $1.0625+02$ & \\
\hline 1 & : & $1.681 E+00$ & $3.043 E+00$ & $5.766 E+00$ & $1.121 E+01$ & $2.211 E \div 01$ & $4.3995 \div 01$ & 8. $747 E+01$ & $1.746 E+02$ & $3.489 E+02$ & \\
\hline 1.2 & : & $1.898 E+\infty 0$ & $3.914 E+00$ & $8.491 E+00$ & $1.894 E+01$ & $4.265 E+01$ & $9.76 \% E+01$ & $2.236 E+02$ & $5.127 E+02$ & 1.17TE+03 & \\
\hline 1.4 & $:$ & $2.161 E+\infty 0$ & $5.104 E+\infty 0$ & 1.270E+01 & 3.251E+01 & 8. $450 E+01$ & $2.213 E+02$ & $5.817 E+02$ & $1.532 E \div 03$ & $4.039 E+03$ & \\
\hline 1.6 & $:$ & $2.480 E+\infty 0$ & $6.727 E+\infty 0$ & $1.923 E+01$ & $5.654 E+01$ & $1.688 E+02$ & $5.077 E+02$ & $1.535 E+03$ & $4.639 E+03$ & $1.405 E+04$ & \\
\hline 1.8 & $:$ & $2.866 E+00$ & $8.94 \%++\infty 0$ & $2.940 E+01$ & $9.934 E+01$ & $3.406 E+02$ & $1.17 \pi+03$ & $4.083 E+03$ & $1.419 E+04$ & $4.9365+04$ & \\
\hline 2 & : & 3. $3335+00$ & 1.200E+01 & $4.532 E+01$ & $1.760 E+02$ & $6.933 E+02$ & 2. $752 E+03$ & 1.097E+04 & $4.378 E+04$ & $1.7496+05$ & \\
\hline
\end{tabular}

$B 1(N, r, w)$ for $r=32$

\begin{tabular}{|c|c|c|c|c|c|c|c|c|c|c|c|}
\hline 4 & $N=$ & 4 & 8 & 16 & 32 & 64 & 128 & 256 & 512 & 1024 & INF \\
\hline 2 & : & $1.000 E+\infty 0$ & $1.000 E+00$ & 1.000E+00 & $1.000 E+\infty$ & $1.000 E+\infty 0$ & $1.000 E+\infty 0$ & 1. $000 E+\infty 0$ & $1.000 E+\infty$ & 1. $000 E+\infty 0$ & $1.000 E+\infty 0$ \\
\hline-1.8 & $:$ & $9.999 E-01$ & $9.999 E-01$ & $9.999 E-01$ & $9.999 E-01$ & $9.999 E-01$ & $9.9905-01$ & $9.998 E-01$ & $9.998 E-01$ & $9.990 E-01$ & $9.998 E-01$ \\
\hline 1.6 & : & $9.998 E-01$ & $9.997 E-01$ & $9.996 E-01$ & $9.996 E-01$ & $9.996 \varepsilon-01$ & $9.995 E-01$ & $9.995 x-01$ & $9.995 E-01$ & $9.995 E-01$ & $9.995 E-01$ \\
\hline 1.4 & : & $9.997 E-01$ & $9.994 E-01$ & $9.993 E-01$ & $9.992 E-01$ & $9.991 E-01$ & $9.991 E-01$ & $9.991 E-01$ & $9.991 E-01$ & $9.991 E-01$ & $9.991 E-01$ \\
\hline 1.2 & : & $9.996 E-01$ & $9.9935-01$ & $9.991 E-01$ & $9.990 E-01$ & $9.9895-01$ & $9.988 c-01$ & $9.988 E-01$ & $9.908 \varepsilon-01$ & $9.988 \varepsilon-01$ & $9.988 \varepsilon-01$ \\
\hline 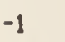 & : & 1.000E+00 & 1. $000 E+00$ & $1.000 E+\infty$ & $1.000 E+\infty 0$ & $1.000 E+\infty$ & 1. $000 E+00$ & 1. $000 E+00$ & $1.000 E+\infty 0$ & 1. $000 E+\infty$ & $1.000 E+\infty 0$ \\
\hline .8 & : & $1.002 E+00$ & $1.003 E+00$ & $1.005 E+\infty 0$ & $1.006 E+00$ & $1.006 E+00$ & 1.007E+00 & $1.007 E+\infty 0$ & 1.007E++00 & $1.007 E+00$ & $1.008 \varepsilon+00$ \\
\hline .6 & $:$ & $1.007 \mathrm{E}+00$ & $1.014 E+00$ & $1.01 \% E+\infty$ & $1.024 E+\infty 0$ & $1.028 E+\infty 0$ & $1.030 E+00$ & $1.032 E+\infty 0$ & $1.033 E+\infty 0$ & $1.034 E+\infty 0$ & $1.036 E+00$ \\
\hline. .4 & : & $1.019 E+00$ & $1.03 \%+00$ & $1.057 E+\infty 0$ & $1.073 E+\infty 0$ & $1.087 E+00$ & $1.098 E+00$ & 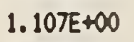 & $1.114 E+\infty 0$ & $1.119 E+\infty$ & $1.136 E+00$ \\
\hline-.2 & : & $1.043 E+00$ & $1.090 E+\infty$ & 1. $139 E+\infty 0$ & $1.186 E+\infty 0$ & $1.230 E+\infty 0$ & $1.271 E+00$ & $1.307 E+\infty$ & $1.3396+\infty 0$ & $1.368 E+00$ & $1.563 E+\infty 0$ \\
\hline 0 & : & & & & & $E+\infty$ & & $1.81 \% E+\infty$ & $57 E+\infty 0$ & $2.095 E+\infty 0$ & \\
\hline .2 & : & $1.146 E+00$ & $1.338 E+\infty 0$ & $1.57 \% E+\infty 0$ & $1.871 E+\infty 0$ & $2.218 E+\infty 0$ & $2.625 E+\infty 0$ & 3.097E+00 & 3. $643 E+\infty 0$ & $4.272 E+\infty 0$ & \\
\hline .4 & : & $1.233 E+\infty$ & $1.572 E+\infty 0$ & $2.046 E+\infty 0$ & $2.693 E+00$ & $3.565 E+00$ & $4.729 E+\infty$ & $6.275 E+\infty$ & 8. $320 E+\infty 0$ & $1.102 E+01$ & \\
\hline .6 & : & $1.348 E+\infty 0$ & $1.908 E+\infty$ & $2.787 E+\infty 0$ & 4.147E+00 & $6.231 E+\infty 0$ & $9.400 E+00$ & 1.424E+01 & $2.158 E+01$ & 3. $270 E+01$ & \\
\hline .8 & : & 1. $494 E+\infty 0$ & $2.378 E+\infty 0$ & $3.940 E+00$ & $6.685 E+00$ & $1.149 E+01$ & $1.986 E+01$ & 3.447E+01 & $5.9925+01$ & $1.042 E+02$ & \\
\hline 1 & : & $1.674 E+\infty 0$ & $3.021 E+\infty$ & $5.716 E+\infty 0$ & $1.111 E+01$ & $2.188 \varepsilon+01$ & $4.344 E+01$ & $8.656 E+01$ & $1.728 E+02$ & 3. $453 E+02$ & \\
\hline 1.2 & : & $1.893 E+\infty 0$ & $3.898 E+\infty 0$ & $8.448 E+00$ & $1.883 E+01$ & $4.261 E+01$ & $9.714 E+01$ & $2.223 E+02$ & 5.097E +02 & $1.170 E+03$ & \\
\hline 1.4 & : & $2.158 E+\infty 0$ & $5.091 E+\infty 0$ & $1.266 E * 01$ & $3.242 E+01$ & $8.425 E+01$ & $2.206 E+02$ & $5.799 E+02$ & $1.527 E+03$ & $4.027 E+03$ & \\
\hline 1.6 & : & $2.478 E+\infty 0$ & $6.71 \%+00$ & $1.920 E+01$ & $5.646 E+01$ & $1.685 E+02$ & $5.070 E+02$ & $1.531 E+03$ & $4.6325+03$ & $1.403 E+04$ & \\
\hline 1.8 & : & $2.865 E+\infty 0$ & $8.945 E+\infty 0$ & $2.938 E+01$ & $9.928 E+01$ & 3. $405 E+02$ & $1.176 E+03$ & 4. $081 E+03$ & $1.418 E+04$ & $4.934 E+04$ & \\
\hline . & : & 3. $333 E+\infty$ & $1.200 E+01$ & $4.533 E+01$ & $1.760 E+02$ & $6.933 E+02$ & $2.752 E+03$ & $1.097 E+04$ & $4.378 E+04$ & 1.749E+05 & \\
\hline
\end{tabular}


$01(N, r$, cus $)$ for $r=64$

\begin{tabular}{|c|c|c|c|c|c|c|c|c|c|c|c|}
\hline 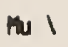 & $N=$ & 4 & 8 & 16 & 32 & 64 & 128 & 256 & 512 & 1024 & INF \\
\hline 2 & : & $1.000 E+\infty 0$ & $1.000 E+\infty$ & $1.000 E+00$ & $1.000 E+00$ & $1.000 E+00$ & $1.000 E+00$ & $1.000 E+\infty 0$ & $1.000 E+00$ & $1.000 E+00$ & 1. $000 E+00$ \\
\hline 1.8 & : & $1.000 E+00$ & $1.000 E+\infty 0$ & 1.000E+CO & $1.000 E+\infty$ & $1.000 E+\infty 0$ & $1.000 E+\infty 0$ & $1.000 E+00$ & $1.000 E+\infty 0$ & $1.000 E+\infty 0$ & $1.000 E+00$ \\
\hline 1.6 & : & $9.999 E-01$ & $9.999 E-01$ & $9.999 E-01$ & 9.999E-01 & $9.999 E-01$ & $9.999 E-01$ & $9.998 E-01$ & $9.998 E-01$ & $9.998 E-01$ & $9.998 E-01$ \\
\hline 1.4 & : & $9.999 E-01$ & $9.99 \% E-01$ & $9.997 E-01$ & $9.997 E-01$ & $9.99 \pi-01$ & $9.997 E-01$ & $9.997 E-01$ & $9.9966-01$ & $9.996 E-01$ & $9.996 E-01$ \\
\hline 1.2 & $:$ & $9.998 E-01$ & $9.997 E-01$ & $9.996 E-01$ & $9.995 E-01$ & $9.995 E-01$ & $9.995 E-01$ & $9.995 E-01$ & $9.995 E-01$ & $9.995 E-01$ & $9.995 E-01$ \\
\hline , & : & $1.000 E+00$ & $1.000 E+\infty 0$ & $1.000 E+\infty 0$ & $1.000 E+\infty 0$ & $1.000 E+00$ & 1. $000 E+00$ & $1.000 E+00$ & 1.000E+00 & 1. $000 E+00$ & $1.000 E+\infty 0$ \\
\hline .8 & : & $1.001 E+00$ & $1.00 x+\infty$ & $1.003 E+\infty$ & $1.003 E+00$ & $1.004 E+\infty 0$ & $1.004 E+\infty$ & $1.004 E+00$ & $1.004 E+\infty 0$ & $1.004 E+\infty 0$ & $1.004 E+\infty 0$ \\
\hline .6 & : & $1.005 E+\infty 0$ & $1.009 E+\infty 0$ & $1.013 E+\infty 0$ & $1.016 E+\infty 0$ & $1.018 E+\infty$ & $1.0 Z: J E+00$ & $1.021 E+\infty 0$ & $1.022 E+00$ & $1.022 E+\infty 0$ & $1.024 E+\infty 0$ \\
\hline-.4 & : & $1.014 E+\infty$ & $1.028 E+\infty 0$ & $1.042 E+\infty$ & $1.054 E+00$ & $1.064 E+00$ & $1.072 E+\infty 00$ & $1.078 E+00$ & $1.083 E+00$ & $1.087 E+\infty 0$ & $1.100 E+00$ \\
\hline 2 & : & $1.035 E+00$ & $1.073 E+00$ & $1.112 x+\infty$ & $1.151 E+\infty 0$ & $1.187 E+\infty 0$ & $1.220 E+00$ & $1.249 E+00$ & $1.275 E+\infty 0$ & $1.298 E+00$ & $E+\infty$ \\
\hline 0 & : & $1.073 E+00$ & $1.161 E+00$ & $1.261 E+00$ & $1.36 \%+00$ & $1.482 E+\infty 0$ & $1.600 E+00$ & $1.71 \%+\infty 0$ & $1.840 E+\infty 0$ & $1.961 E+00$ & \\
\hline .2 & $:$ & $1.135 E+00$ & $1.313 E+\infty$ & $1.536 E+00$ & $1.808 E+00$ & $2.129 E+00$ & $2.506 E+00$ & $2.944 E+00$ & $3.450 E+00$ & $4.033 E+00$ & \\
\hline .4 & : & $1.224 E+00$ & $1.548 E+\infty 0$ & $2.003 E+\infty 0$ & $2.624 E+\infty 00$ & $3.461 E+00$ & $4.578 E+\infty 00$ & $6.060 E+00$ & $8.023 E+00$ & $1.062 E+01$ & \\
\hline .6 & : & $1.341 E+00$ & $1.889 E+00$ & $2.749 E+\infty 0$ & $4.000 E+00$ & $6.119 E+00$ & $9.229 E+00$ & $1.396 E+01$ & $2.114 E+01$ & 3. $203 E+01$ & \\
\hline .8 & $:$ & $2.489 E+00$ & $2.363 E+\infty 0$ & $3.909 E+00$ & $6.624 E+\infty 0$ & $1.137 E+01$ & $1.966 E+01$ & $3.411 E+01$ & $5.92 \% \Leftarrow+01$ & $1.031 E+02$ & \\
\hline 1 & $:$ & $1.670 E+\infty 0$ & $3.010 E+\infty 0$ & $5.691 E+\infty 0$ & $1.105 E+01$ & $2.1 \pi T E+01$ & $4.322 E+01$ & $8.611 E+01$ & $1.719 E+02$ & $3.435 E+02$ & \\
\hline 1.2 & : & $1.890 E+00$ & $3.891 E+\infty 0$ & $8.429 E+00$ & $1.87 \% E \div 01$ & $4.251 E+01$ & $9.690 E+01$ & $2.218 E+02$ & $5.005 E+02$ & $1.16 \pi E+03$ & \\
\hline 1.4 & : & $2.156 E+00$ & $5.08 \pi E+00$ & $1.265 E+01$ & 3. $230 E+01$ & $8.415 E+01$ & $2.204 E+02$ & 5. $793 E+02$ & $1.526 E+03$ & $4.022 E+03$ & \\
\hline 1.6 & : & $2.47 \pi+\infty$ & $6.716 E+00$ & $1.91 \% E+01$ & $5.644 E+01$ & $1.685 E+02$ & $5.067 \xi+02$ & $1.530 E+03$ & $4.630 E \div 03$ & $1.402 E+04$ & \\
\hline 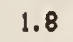 & : & $2.865 E+\infty$ & $8.943 E+00$ & $2.938 E+01$ & $9.92 \pi+01$ & $3.404 E+02$ & $1.176 E+03$ & 4. $080 E+03$ & $1.418 E+04$ & $4.933 E+04$ & \\
\hline . & : & $3.3335+\infty 0$ & 1. $200 E+01$ & $4.533 E+01$ & $1.760 E+02$ & $6.933 E+02$ & $2.75 x E+03$ & $1.097 E+04$ & $4.378 E+04$ & $1.749 E+05$ & \\
\hline
\end{tabular}

$B 1(N, r, m)$ for $r=128$

\begin{tabular}{|c|c|c|c|c|c|c|c|c|c|c|c|}
\hline 1 & $N=$ & 4 & 8 & 16 & 32 & 64 & 128 & 256 & 512 & 1024 & INF \\
\hline 2 & $:$ & $1.000 E+\infty$ & $1.000 E+00$ & $1.000 E+\infty 0$ & $1.000 E+00$ & $1.000 E+\infty 0$ & $1.000 E+\infty 0$ & $1.000 E+\infty 0$ & 1. $000 E+00$ & $1.000 E+00$ & 1. $000 E+\infty$ \\
\hline 1.8 & : & $1.000 E+00$ & $1.000 E+00$ & $1.000 E+00$ & $1.000 E+00$ & $1.000 E+00$ & $1.000 E+\infty 0$ & $1.000 E+\infty 0$ & 1. $000 E+00$ & $1.000 E+00$ & $1.000 E+00$ \\
\hline 1.6 & : & $1.000 E+00$ & 1. $000 E+\infty 0$ & $1.000 E+00$ & $1.000 E+00$ & $1.000 E+\infty 0$ & $1.000 E+00$ & $9.999 E-01$ & $9.999 E-01$ & $9.999 E-01$ & $9.9995-01$ \\
\hline 1.4 & : & $1.000 E+\infty$ & $9.999 E-01$ & $9.999 E-01$ & $9.999 E-01$ & $9.999 E-01$ & $9.999-01$ & $9.999 E-01$ & $9.999 E-01$ & $9.999 E-01$ & $9.999 E-01$ \\
\hline 1.2 & : & $9.999 E-01$ & $9.999 E-01$ & $9.998 E-01$ & $9.998 E-01$ & $9.998 E-01$ & $9.998 E-01$ & $9.998 E-01$ & $9.998 E-01$ & $9.998 E-01$ & $9.998 E-01$ \\
\hline 1 & : & $1.000 E+\infty 0$ & $1.000 E+\infty$ & $1.000 E+00$ & 1.000E+00 & $1.000 E+\infty 0$ & $1.000 E+\infty 0$ & $1.000 E+\infty 0$ & $1.000 E+00$ & $1.000 E+\infty 0$ & 1. $000 E+\infty 0$ \\
\hline .8 & : & $1.001 E+00$ & $1.001 E+\infty$ & $1.00 \mathbb{E}+00$ & $1.00 x E+\infty$ & $1.002 E+\infty 0$ & $1.002 E+00$ & $1.00 x+\infty$ & $1.002 E+\infty$ & $1.00 X E+\infty 0$ & $1.002 E+00$ \\
\hline 6 & $:$ & $1.0035+00$ & $1.006 E+00$ & $1.008 E+00$ & $1.010 E+\infty 0$ & $1.012 E+\infty 0$ & $1.013 E+00$ & $1.014 E+00$ & $1.014 E+00$ & $1.015 E+\infty 0$ & $1.015 E+00$ \\
\hline 4 & $:$ & $1.010 E+\infty$ & $1.021 E+\infty$ & $1.031 E+00$ & 1. $040 E+00$ & $1.04 \pi+\infty 0$ & $1.053 E+00$ & $1.058 E+\infty 0$ & $1.062 \pm+00$ & $1.065 E+\infty 0$ & $1.074 E+\infty 0$ \\
\hline .2 & : & $1.02 \%+\infty 00$ & $1.060 E+\infty 0$ & $1.092 \leq+\infty 0$ & $1.124 E+00$ & $1.153 E+\infty 0$ & $1.181 E+\infty$ & $1.205 E+\infty 0$ & $1.226 E+\infty$ & $1.245 E+00$ & $1.375 E+00$ \\
\hline 0 & : & $1.065 E+00$ & $1.144 E+\infty 0$ & $1.232 E+00$ & $1.329 E+00$ & $1.430 E+\infty 0$ & $1.534 E+\infty 0$ & $1.640 E+00$ & $1.748 E+00$ & $1.856 E+00$ & \\
\hline .2 & : & $1.127 E+\infty$ & $1.294 E+\infty 0$ & $1.5045+\infty 0$ & $1.75 \%+\infty$ & $2.082 E+00$ & $2.416 E+00$ & $2.827 E+\infty 0$ & 3. $303 E+\infty 0$ & $3.851 E+\infty$ & \\
\hline .4 & : & $1.217 E+\infty$ & $1.532 E+00$ & $1.973 E+00$ & $2.576 E+00$ & 3. $388 \varepsilon+00$ & 4. $471 E+\infty$ & $5.909 E+00$ & $7.813 E+00$ & $1.033 E+01$ & \\
\hline .6 & : & $1.336 E+00$ & $1.877 E+\infty$ & $2.725 E+00$ & $4.03 \pi+00$ & $6.048 E+\infty 0$ & $9.115 E+00$ & $1.378 \varepsilon+01$ & $2.086 E+01$ & $3.160 E+01$ & \\
\hline .8 & : & $1.486 E+00$ & $2.354 E+00$ & $3.891 E+00$ & $6.589 E+\infty 0$ & 1. $131 E+01$ & $1.955 E+01$ & $3.391 E+01$ & $5.893 E+01$ & $1.025 E+02$ & \\
\hline 1 & : & $1.668 E+00$ & $3.005 E+0.0$ & $5.679 E+\infty 0$ & $1.103 E+01$ & 2. $17 Z E+01$ & $4: 311 E+01$ & $8.58 \% E+01$ & $1.714 E+02$ & $3.426 E+02$ & \\
\hline 1.2 & : & $1.889 E+\infty 0$ & $3.887 t+\infty 0$ & $8.421 E+\infty 0$ & $1.877 E+01$ & $4.246 E+01$ & $9.680 E+01$ & $2.215 E+02$ & $5.079 \varepsilon+02$ & $1.166 E+03$ & \\
\hline 1.4 & : & $2.156 E+\infty 0$ & $5.005 E+00$ & $1.265 E+01$ & 3.237E+01 & $8.411 E+01$ & $2.203 E+02$ & $5.790 E+02$ & $1.525 E+03$ & $4.021 E+03$ & \\
\hline 1.6 & : & $2.477 E+\infty$ & $6.7155+\infty 0$ & $1.91 \% \Leftarrow+01$ & $5.643 E+01$ & $1.684 E+02$ & $5.06 \pi+02$ & $1.530 E+03$ & $4.629 E+03$ & $1.402 E+04$ & \\
\hline 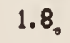 & : & $2.865 E+10$ & $8.913 E+00$ & $2.938 E+01$ & $9.926 E+01$ & 3. 404E +02 & $1.176 E+03$ & $4.060 E+03$ & $1.418 E+04$ & $4.932 E+04$ & \\
\hline 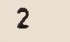 & : & $3.333 E+\infty 0$ & 1.200E+01 & $4.533 E+01$ & $1.760 E+02$ & $6.933 E+02$ & $2.75 x+03$ & $1.09 \pi+04$ & $4.378 \varepsilon+04$ & $1.749 E+05$ & \\
\hline
\end{tabular}


$B 1(N, r$, eus $)$ for $r=256$

\begin{tabular}{|c|c|c|c|c|c|c|c|c|c|c|c|}
\hline Nu 1 & $N=$ & 4 & 8 & 16 & 32 & 64 & 128 & 256 & 512 & 1024 & INF \\
\hline-2 & $:$ & $1.000 E+00$ & $1.000 E+00$ & $1.000 E+00$ & 1.000E+00 & $1.000 E+\infty 0$ & $1.000 E+00$ & 1.000E+00 & 1. $000 E+\infty 0$ & $1.000 E+00$ & 1.000E+00 \\
\hline-1.8 & : & $1.000 E+00$ & $1.000 E+00$ & $1.000 E+00$ & $1.000 E+00$ & $1.000 E+\infty 0$ & $1.000 E+00$ & $1.000 E+\infty 0$ & 1.000E+00 & $1.000 E+00$ & $1.000 E+00$ \\
\hline-1.6 & : & 1. $000 E+00$ & $1.000 E+00$ & $1.000 E+00$ & 1.000E+CO & $1.000 E+\infty 0$ & $1.000 E+\infty 0$ & $1.000 E+\infty 0$ & 1.000E+CO & $1.000 E+00$ & 1.000E+CO \\
\hline-1.4 & : & $1.000 E+\infty 0$ & $1.000 E+\infty 0$ & 1.000E+CO & $1.000 E+\infty 0$ & $1.000 E+\infty 0$ & $1.000 E+00$ & $1.000 E+\infty 0$ & $9.999-01$ & $9.999 E-01$ & $9.999 E-01$ \\
\hline-1.2 & : & $1.000 E+00$ & $9.999 E-01$ & $9.999 E-01$ & $9.999 E-01$ & $9.999 E-01$ & $9.9995-01$ & $9.999-01$ & $9.999 E-01$ & $9.9995-01$ & $9.9995-01$ \\
\hline-1 & $:$ & $1.000 E+00$ & $1.000 E+00$ & $1.000 E+00$ & $1.000 E+\infty 0$ & $1.000 E+00$ & $1.000 E+\infty 0$ & $1.000 E+00$ & $1.000 E+\infty 0$ & $1.000 E+00$ & 1.000E+Co \\
\hline-.8 & : & $1.000 E+00$ & $1.001 E+00$ & $1.001 E+\infty 0$ & $1.001 E+\infty 0$ & $1.001 E+00$ & $1.001 E+\infty$ & $1.001 E+\infty$ & $1.001 E+\infty 0$ & $1.001 E+\infty$ & $1.001 E+\infty$ \\
\hline-.6 & : & $1.002 E+00$ & 1.004E+00 & $1.005 E+00$ & $1.007 E+\infty 0$ & $1.008 E+00$ & $1.008 E+\infty 00$ & 1.00\% +00 & 1.00\%E+C0 & $1.010 E+\infty$ & $1.010 E+\infty$ \\
\hline-.4 & : & $1.008 E+00$ & $1.016 E+00$ & $1.023 E+\infty 0$ & $1.029 E+00$ & $1.035 E+\infty 0$ & $1.039 E+\infty 0$ & $1.043 E+\infty 0$ & $1.046 E+\infty 0$ & $1.048 E+\infty 0$ & $1.055 E+\infty 0$ \\
\hline-.2 & : & $1.024 E+\infty 0$ & $1.050 E+00$ & $1.077 E+\infty 0$ & $1.103 E+00$ & $1.127 E+\infty 0$ & $1.150 E+00$ & $1.170 E+\infty 0$ & $1.188 E+\infty 0$ & $1.204 E+\infty 0$ & $1.311 E+\infty$ \\
\hline 0 & : & $1.059 E+00$ & $1.130 E+00$ & $1.210 E+\infty 0$ & $1.296 E+00$ & $1.388 E+00$ & $1.482 E+\infty 0$ & $1.577 E+\infty$ & $1.674 E+\infty 0$ & $1.772 E+\infty$ & \\
\hline .2 & : & $1.121 E+00$ & $1.280 E+00$ & $1.479 E+00$ & $1.722 E+00$ & $2.009 E+00$ & $2.346 E+\infty 00$ & $2.737 E+\infty 0$ & $3.18 \% E+\infty 0$ & $3.710 E+\infty$ & \\
\hline .4 & : & $1.212 \varepsilon+\infty 0$ & $1.520 E+\infty 0$ & $1.952 E+00$ & $2.541 E+\infty 0$ & $3.335 E+00$ & 4. $394 E+00$ & $5.800 E+00$ & $7.662 E+\infty 0$ & $1.012 E+01$ & \\
\hline .6 & : & $1.333 E+\infty 0$ & $1.869 E+00$ & 2. $709 E+00$ & $4.010 E+\infty 0$ & $6.003 E+\infty 0$ & $9.042 E+\infty 0$ & $1.366 E+01$ & $2.068 E+01$ & 3. 132E+01 & \\
\hline .8 & $:$ & $1.484 E+00$ & $2.350 E+\infty 0$ & $3.881 E+\infty 0$ & $6.570 E+00$ & 1.127E+01 & $1.948 E+01$ & $3.380 E+01$ & $5.873 E+01$ & $1.022 E+02$ & \\
\hline 1 & $:$ & $1.668 E+\infty 0$ & $3.003 E+00$ & $5.6735+00$ & $1.101 E+01$ & $2.169 E+01$ & $4.305 E+01$ & $8.578 E+01$ & $1.712 E+02$ & $3.421 E+02$ & \\
\hline 1.2 & $:$ & $1.889 \varepsilon+00$ & $3.886 E+00$ & $8.41 \varepsilon \varepsilon+\infty$ & $1.876 E+01$ & $4.244 E+01$ & $9.675 E+01$ & $2.214 E+02$ & $5.0 T 7 E+02$ & $1.165 E+03$ & \\
\hline 1.4 & : & $2.156 E+\infty 0$ & $5.084 E+00$ & $1.264 E+(11$ & $3.236 E+01$ & $8.410 E+01$ & $2.202 E+02$ & $5.789 E+02$ & $1.525 E+03$ & $4.020 E+03$ & \\
\hline 1.6 & : & $2.477 E+00$ & $6.715 E+\infty$ & $1.919 E+01$ & $5.642 E+01$ & $1.684 E+02$ & $5.066 E+02$ & $1.530 E+03$ & $4.629 E+03$ & $1.402 E+04$ & \\
\hline 1.8 & : & $2.865 E+00$ & 8. $943 E+(00)$ & $2.938 E+01$ & $9.926 E+01$ & $3.404 E+02$ & $1.176 E+03$ & $4.080 E+03$ & $1.418 E+04$ & $4.932 E+04$ & \\
\hline 2 & $:$ & 3. $333 E+00$ & $1.200 E+01$ & $4.533 E+01$ & $1.760, E+02$ & $6.933 E+02$ & $2.752 E+03$ & $1.097 E+04$ & $4.378 E+04$ & $1.749 E+05$ & \\
\hline
\end{tabular}

B1 $(N, r$, mu $)$ for $r=512$

\begin{tabular}{|c|c|c|c|c|c|c|c|c|c|c|c|}
\hline Ma 1 & $N=$ & 4 & 8 & 16 & 32 & 64 & 128 & 256 & 512 & 1024 & INF \\
\hline-2 & : & $1.0005+\infty 0$ & $1.000 \varepsilon+\infty$ & 1.000E+00 & $1.000 E+00$ & $1.000 E+\infty 0$ & $1.000 E+00$ & $1.000 E+\infty 0$ & $1.000 E+00$ & $1.000 E+00$ & 1. $000 E+00$ \\
\hline-1.8 & : & $1.000 E+\infty 0$ & $1.000 E+\infty$ & $1.000 E+00$ & $1.000 E+00$ & $1.000 E+\infty 0$ & $1.000 E+00$ & 1. $000 E+00$ & $1.000 E+\infty 0$ & $1.000 E+00$ & $1.000 E+00$ \\
\hline-1.6 & : & $1.000=+00$ & 1.000E+00 & $1.000 E+00$ & 1. $000 E+00$ & $1.000 E+00$ & $1.000 E+00$ & $1.000 E+\infty 0$ & $1.000 E+00$ & $1.000 E+\infty 0$ & $1.000 E+00$ \\
\hline-1.4 & : & $1.000 E+00$ & 1. $000 E+00$ & $1.000 E+00$ & $1.000 E+100$ & $1.000 E+00$ & $1.000 E+00$ & $1.000 E+00$ & $1.000 E+00$ & $1.000 E+00$ & $1.000 E+(00)$ \\
\hline-1.2 & $:$ & $1.000 E+00$ & 1.000E+00 & $1.000 E+00$ & 1.000E+00 & $1.000 E+00$ & 1. $000 E+\infty 0$ & $1.000 E+\infty$ & $1.000 E+\infty 0$ & 1.000E $+\infty$ & $1.000 E+00$ \\
\hline-1 & : & 1.000E+D0 & $1.000 E+\infty 0$ & $1.000 E+\infty 0$ & $1.000 E+00$ & 1.000E+00 & $1.000 E+00$ & 1. $000 E+O 0$ & $1.000 E+00$ & $1.000 E+00$ & $1.000 E+(00)$ \\
\hline-.8 & : & $\varepsilon+\infty$ & 1. $000 E+00$ & $1.001 E+\infty$ & $1.001 E+\infty$ & 1.001 & $1.001 E+\infty$ & $1.001 E+\infty$ & $1.001 E+\infty 0$ & $E+\infty$ & $1.001 E+00$ \\
\hline-.6 & $:$ & $1.001 E+00$ & $1.003 E+\infty 0$ & $1.004 E+00$ & $1.004 E+100$ & $1.005 E+00$ & $1.006 E+00$ & $1.006 E+\infty$ & $1.006 E+\infty 0$ & $1.006 E+\infty 0$ & $1.007 E+00$ \\
\hline-.4 & $:$ & 1. $005 E+00$ & $1.012 E+00$ & $1.017 E+00$ & $1.022 E+\infty 0$ & $1.026 E+00$ & $1.030 E+\infty 0$ & $1.032 E+00$ & $1.034 E+00$ & $1.036 E+00$ & $1.041 E+00$ \\
\hline-.2 & : & $1.020 E+\infty 0$ & $1.042 E+\infty 0$ & $1.064 E+00$ & $1.086 E+00$ & $1.107 E+00$ & $1.125 E+00$ & $1.142 E+00$ & $1.157 E+\infty 0$ & $1.170 E+\infty 0$ & $1.261 E+00$ \\
\hline 0 & $:$ & $1.054 E+00$ & $1.118 E+\infty 0$. & $1.191 E+\infty 0$ & $1.270 E+\infty 0$ & $1.353 E+\infty 0$ & $1.438 E+00$ & $1.526 E+\infty 0$ & $1.614 E+00$ & $1.703 E+100$ & \\
\hline .2 & $:$ & 1.1 & +100 & $+\infty$ & $1.692 E$ & & $+\infty$ & $+\infty$ & $\varepsilon+\infty$ & $3.598 E+00$ & \\
\hline .4 & $:$ & $1.209 E+00$ & $1.512 E+00$ & $1.936 E+00$ & $2.516 E+00$ & $3.296 E+00$ & $4.338 E+00$ & $5.721 E+00$ & $7.553 E+\infty 0$ & $9.973 E+00$ & \\
\hline .6 & : & $1.331 E+\infty 0$ & $1.864 E+\infty 0$ & $2.699 E+00$ & $3.992 E+00$ & $5.973 E+00$ & $8.994 E+00$ & 1. $359 E+01$ & $2.056 E+01$ & $3.114 E+01$ & \\
\hline .8 & : & $1.48 .3 E+60$ & 2.347E+CO & $3.875 E+\infty 0$ & $6.558 E+00$ & $1.125 E+01$ & $1.945 E+01$ & $3.373 E+01$ & $5.862 E+01$ & $1.020 E+02$ & \\
\hline 1 & $:$ & $1.667 E+\infty 0$ & $3.001 E+\infty 0$ & $5.670 E+00$ & $1.101 E+01$ & $2.168 E+01$ & 4. $303 E+01$ & $8.572 E+01$ & $1.711 E+02$ & $3.419 E+02$ & \\
\hline 1.2 & $:$ & $1.889 E+C 0$ & $3.8 \operatorname{csE}+00$ & $8.416 E+C 0$ & $1.876 E+01$ & $4.243 E+01$ & $9.673 E+01$ & $2.214 E+02$ & $5.076 E+1) 2$ & $1.165 E+03$ & \\
\hline 1.4 & $:$ & $2.156 E+00$ & $5.084 E+\infty 0$ & $1.264 E+01$ & 3. $236 E+01$ & $8.410 E+01$ & $2.202 E+02$ & $5.789 E+02$ & $1.525 E+03$ & $4.020 E+03$ & \\
\hline 1.6 & : & $2.477 E+00$ & $6.714 E+00$ & $1.91 \%+01$ & $5.642 E+01$ & $1.684 E+02$ & $5.066 E+02$ & $1.530 E+03$ & $4.628 E+03$ & $1.402 E+04$ & \\
\hline 1.8 & $:$ & $2.865 E+\infty 0$ & $8.943 E+00$ & $2.9385+11$ & $9.926 E+01$ & 3. $404 E+02$ & $1.176 E+03$ & $4.080 E+03$ & $1.418 E+04$ & $4.932 E+04$ & \\
\hline 2 & : & $3.333 E+10$ & 1.200E+01 & $4.5535+01$ & $1.760 E+02$ & $6.933 E+02$ & 2. $752 E+03$ & $1.097 E+04$ & $4.378 E+04$ & $1.749 E+05$ & \\
\hline
\end{tabular}


$B 1(K, r$, wu $)$ for $r=1024$

\begin{tabular}{|c|c|c|c|c|c|c|c|c|c|c|c|}
\hline Hu 1 & $\omega$ & 4 & 8 & 16 & 32 & 64 & 128 & 256 & 512 & 1024 & In \\
\hline-2 & 8 & $1.000 E+\infty 0$ & $1.000<+\infty 0$ & $1.000 E+00$ & $1.000 E+\infty 0$ & 1.000E+00 & $1.000 E+00$ & $1.000 E+00$ & $1.000 E+\infty$ & $1.000 E+\infty 0$ & $1.000 E+00$ \\
\hline-1.8 & 8 & $1.000<+00$ & $1.000 E+00$ & $1.000 E+00$ & $1.000 E+00$ & $1.0005+00$ & $1.000 E+00$ & $1.000 E+00$ & 1.000E & $1.000 E+\infty 0$ & $1.000=200$ \\
\hline-1.6 & 8 & $1.000 \leqslant+00$ & $1.000 E+\infty 0$ & $1.000 E+00$ & $1.000 E+00$ & $1.000 E+\infty 0$ & $1.000 E+00$ & $1.000 E+00$ & 1.000E $\div 00$ & $1.000 E+00$ & $1.000 E+00$ \\
\hline-1.4 & 8 & $1.000 E+00$ & $1.000 E+\infty 0$ & $1.000 E+00$ & $1.000 E+00$ & $1.000 E+00$ & $1.000 E+00$ & $1.000 E+00$ & $1.000 E+\infty 0$ & $1.000 E+\infty 0$ & 1. $000 E+\infty$ \\
\hline-1.2 & 8 & $1.000 \leqslant+00$ & $1.000 E+00$ & $1.000 E+00$ & $1.000 E+00$ & $1.0005+00$ & $1.000=+00$ & $1.000 E+00$ & $1.000 E+00$ & $1.000 E+00$ & 1. $000 E+\infty 0$ \\
\hline-1 & 1 & 1. $000 E+00$ & $1.000=+00$ & $1.000 E+\infty 0$ & $1.000=+00$ & $1.000 E+\infty 0$ & $1.000 E+00$ & $1.000 E+00$ & $1.000 E+00$ & $1.000 E+\infty 0$ & 1.000E+00 \\
\hline-.8 & 8 & $1.000 E+00$ & $1.000 E+00$ & $1.000 E+\infty 0$ & $1.000 E+00$ & $1.000 E+00$ & $1.000 E+00$ & $1.000 E+00$ & $1.000 E+\infty 0$ & $00 E+\infty 0$ & 1.00 \\
\hline-.6 & 1 & $1.001 E+00$ & $1.0028+00$ & $1.002 \leq+00$ & $1.0085+00$ & $1.00<5+\infty 0$ & $1.004 E+\infty 0$ & $1.004 E+00$ & $1.004 E+00$ & $1.004 E+00$ & $1.004 E+00$ \\
\hline-.4 & 8 & $1.0045+\infty 0$ & 1.00:E+00 & 1.0135 & & 1.0205 & $1.022 x$ & $1.024 E+00$ & $1.026 E+00$ & $1.02 \pi+\infty 0$ & $1.031 E+00$ \\
\hline-.2 & 8 & $1.01 \pi E+\infty 0$ & $1.08=5+00$ & $1.051 E+00$ & $1.0735+00$ & $1.090 E+\infty 0$ & $1.106 E+00$ & 1. $120 \leq+00$ & $1.132 x+00$ & $1.144 E+00$ & $1 . x$ \\
\hline 0 & 1 & $1.04 \%+00$ & $1.100 E+00$ & $1.175 E+00$ & $1.2465+00$ & $1.324 E+00$ & $1.402 \leq+00$ & $1.485 \leq i 00$ & $1.564 E+00$ & $1.645 E+00$ & \\
\hline .2 & 8 & $1.112 \geq+00$ & $1.2005+00$ & $1.44 E+\infty 0$ & $1.668 E+00$ & $1.934 E+00$ & $2.245 E+00$ & $2.60 \pi$ & $3.025 E+00$ & $3.50 \pi+\infty 0$ & \\
\hline .4 & 8 & $1.2065+00$ & $1.500=00$ & $1.924 E+00$ & $2.497 E+00$ & $3.26 \div \varepsilon+00$ & $4.29 \pi+00$ & $5.663 \div+00$ & $7.472<+\infty 0$ & $9.86 x+00$ & \\
\hline .6 & 8 & 1.30 & $1.860 E+00$ & $2.698 \leqslant+00$ & $3.900 E+00$ & $5.9535+\infty 0$ & $8.963 E+00$ & $1.354 E+01$ & 2.04\%+01 & $3.102 \varepsilon+01$ & \\
\hline .8 & 1 & $1.482 \pi+00$ & $2.345 E+00$ & $3.872 \div+00$ & $6.5525+00$ & $1.124 E+01$ & $1.942 x+01$ & $3.36 \% 5+01$ & 5. $855 E+01$ & $1.018 \varepsilon+02$ & \\
\hline 1 & $:$ & $1.66 \pi+00$ & $3.001 E+00$ & $5.6685+00$ & $1.100 E+01$ & $2.16 \pi+01$ & $4.301 E+01$ & 8.56\%+01 & $1.711 E+02$ & $3.418 E+02$ & \\
\hline 1.2 & 8 & $1.8095+00$ & $3.865 E+00$ & $8.416 E+00$ & $1.876 E+01$ & $4.243 E+01$ & $9.6722+01$ & $2.213 E+02$ & $5.075 E+02$ & $1.165 E+03$ & \\
\hline 1.4 & 8 & $2.156 ̈++00$ & $5.084 E+\infty 0$ & $1.264 E+01$ & $3.236 E+01$ & $8.409 \varepsilon+01$ & $2.202 \leq+02$ & $5.769 €+02$ & $1.525 E+03$ & $4.020 E+03$ & \\
\hline 1.6 & 8 & $2.47 \pi+00$ & $6.714 E+\infty 0$ & $1.919 E+01$ & $5.642 x+01$ & $1.694 E+02$ & $5.066 E+02$ & $1.530 \varepsilon+03$ & $4.628 E+03$ & 1. $402 E+04$ & \\
\hline 1.8 & 1 & $2.865 E+\infty 0$ & $8.943 E+\infty 0$ & $2.938 E+01$ & $9.926 E+01$ & 3. $404 E+02$ & $1.176 E+03$ & $4.000 E+03$ & $1.416 e+04$ & $4.932 E+04$ & \\
\hline 2 & : & $3.33<E+60$ & $1.200 E+01$ & $4.533 E+01$ & $1.760 E+02$ & $6.933 E+02$ & $2.73 x+03$ & $1.097 E+04$ & $4.378 E+04$ & $1.749 E+05$ & \\
\hline
\end{tabular}

$B 1(N, r, m)$ for $r=2048$

\begin{tabular}{|c|c|c|c|c|c|c|c|c|c|c|c|}
\hline 4 & $N=$ & 4 & 8 & 16 & 32 & 64 & 128 & 256 & 512 & 1024 & INF \\
\hline-2 & : & 1.000E+00 & 1. $000 E+\infty$ & $1.000 E+\infty 0$ & $1.000 E+00$ & $1.000 E+00$ & $1.000 E+\infty$ & $1.000 E+\infty 0$ & $1.000 E+\infty 0$ & $1.000 E+\infty 0$ & 1. $000 E+\infty$ \\
\hline-1.8 & : & $1.000 E+00$ & 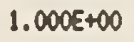 & $1.000 E+\infty 00$ & $1.000 E+\infty 00$ & $1.000 E+00$ & $1.000 E+00$ & $1.000 E+\infty 0$ & $1.000 E+\infty$ & $1.000 E+\infty 0$ & $1.000 E+00$ \\
\hline 1.6 & : & $1.000 E+00$ & $1.000 E+\infty$ & $1.000 E+\infty$ & $1.000 E+\infty$ & $1.000 E+00$ & $1.000 E+00$ & $1.000 E+00$ & 1. $000 E+\infty$ & $1.000 E+\infty$ & $1.000 E+\infty 0$ \\
\hline 1.4 & : & $1.000 E+\infty 0$ & $1.000 E+\infty 0$ & $1.000 E+\infty 0$ & $1.000 E+\infty 0$ & $1.000 \varepsilon+00$ & $1.000 E+\infty 0$ & $1.000 E+00$ & $1.000 E+\infty 0$ & $1.000 E+00$ & $1.000 E+00$ \\
\hline-1.2 & : & $1.000 E+00$ & 1.000E $+\infty 0$ & 1.000E+00 & 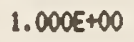 & $1.000 E+\infty 0$ & $1.000 E+\infty 0$ & $1.000 E+\infty 0$ & $1.000 E+\infty 0$ & $1.000 E+\infty$ & $1.000 E+00$ \\
\hline 8 & : & $1.000 E+00$ & $1.000 E+\infty 0$ & 1. $000 E+\infty$ & $1.000 E+\infty 0$ & $1.000 E+00$ & $1.000 E+\infty$ & $1.000 E+00$ & $1.000 E+\infty 0$ & $1.000 E+\infty 0$ & $1.000 E+00$ \\
\hline-.8 & : & $1.000 E+\infty$ & $1.000 E+\infty 0$ & $1.000 E+\infty$ & 1. $000 E+\infty$ & $1.000 E+\infty$ & $1.000 E+\infty 0$ & $1.000 E+\infty 0$ & $1.000 E+\infty$ & $1.000 E+\infty 0$ & 1.000E+00 \\
\hline .6 & : & $1.001 E+\infty 0$ & $1.001 E+\infty 0$ & $1.002 \leq \div 00$ & $1.002 x+\infty 0$ & $1.002 E+00$ & $1.002 E+\infty$ & $1.003 E+00$ & $1.003 E+00$ & $1.003 E+00$ & $1.003 E+00$ \\
\hline. .4 & : & $1.003 E+00$ & $1.007 E+00$ & $1.010 E+\infty$ & $1.012 x+\infty 0$ & $1.015 E+00$ & $1.017 E+\infty$ & $1.018 E+\infty$ & $1.019 E+\infty$ & $1.020 E+\infty 0$ & $1.023 E+00$ \\
\hline-.2 & : & $1.014 E+00$ & $1.030 E+\infty 0$ & $1.046 E+00$ & $1.061 E+\infty$ & $1.076 E+00$ & $1.009 E+00$ & $1.101 E+\infty 0$ & $1.112 E+\infty$ & $1.122 E+\infty 0$ & $1.186 E+00$ \\
\hline 0 & : & $1.045 E+00$ & $1.100 E+\infty 0$ & $1.162 E+\infty 0$ & $1.22 \%+\infty 00$ & $1.299 E+00$ & $1.372 E+\infty 0$ & $1.446 E+\infty$ & $1.521 E+\infty 0$ & $1.596 E+\infty 0$ & \\
\hline .2 & : & & $+\infty 0$ & $1.431 E+\infty$ & $1.648 E+\infty 0$ & $1.906 E+\infty$ & $2.205 \leq+\infty$ & $2.560 E+\infty 0$ & $2.966 E+00$ & & \\
\hline .4 & 8 & $1.204 E+00$ & $1.501 E+\infty$ & $1.916 E+\infty 0$ & $2.483 E+\infty$ & $3.247 E+\infty 0$ & $4.2665+\infty 00$ & $5.620 E+\infty 0$ & $7.412 \varepsilon+\infty$ & $9.780 E+00$ & \\
\hline .6 & : & $1.32 \% E+00$ & $1.858 \varepsilon+00$ & $2.668 E+\infty 0$ & 3.972 & 5.94 & $8.942 E+00$ & 1.351E+01 & $2.044 E+01$ & $3.095 E+01$ & \\
\hline .8 & : & $1.48 x+\infty 0$ & $2.345 E+00$ & $3.870 E+00$ & $6.548 E+\infty 0$ & $1.123 E+01$ & $1.941 E+01$ & 3.367E+01 & $5.851 E+01$ & $1.018 E+02$ & \\
\hline 1 & : & $1.66 \pi E+\infty 0$ & 3. $000 E+00$ & $5.667 \pi+\infty 0$ & $1.100 E+01$ & $2.167 E+01$ & 4. $301 E+01$ & $8.568 E+01$ & $1.710 E+02$ & 3. $417 E+02$ & \\
\hline 1.2 & : & $1.889+\infty+0$ & $3.885 E+00$ & $8.415 E+00$ & $1.875 E+01$ & $4.243 E+01$ & $9.672 E+01$ & 2. $213 \mathrm{E}+02$ & $5.075 E+02$ & $1.165 \mathrm{E}+03$ & \\
\hline 1.4 & : & $2.156 E+\infty 0$ & $5.084 E+\infty 0$ & $1.264 E+01$ & $3.236 E+01$ & 8.409E+01 & $2.202 \Sigma+02$ & 5. $789 E+02$ & $1.525 E+03$ & $4.020 E+03$ & \\
\hline 1.6 & : & $2.477 E+00$ & $6.714 E+\infty 0$ & $1.91 \%+01$ & $5.64 X E+01$ & $1.684 E+02$ & $5.066 E+02$ & $1.530 E+03$ & $4.626 \varepsilon+03$ & $1.402 E+04$ & \\
\hline 1.8 & : & $2.865 E+\infty 00$ & $8.943 E+00$ & $2.9385+01$ & $9.926 E+01$ & 3. 404E+02 & $1.176 E+03$ & $4.080 E+03$ & $1.418 E+04$ & $4.932 E+04$ & \\
\hline 0 & : & $3.333 E+\infty 0$ & $1.200 E+01$ & $4.533 E+01$ & $1.760 E+02$ & $6.933 E+02$ & $2.752 E+03$ & $1.097 E+04$ & $4.378 E+04$ & $1.749 E+05$ & \\
\hline
\end{tabular}


$B I(N, r, w)$ for $r=$ INFINITY

\begin{tabular}{|c|c|c|c|c|c|c|c|c|c|c|c|}
\hline u 1 & $N=$ & 4 & 8 & 16 & 32 & 64 & 128 & 256 & 512 & 1024 & $I N F$ \\
\hline-2 & $:$ & 1. $000 E+00$ & $1.000 E+00$ & $1.000 E+\infty 0$ & $1.000 E+00$ & $1.000 E+00$ & $1.000 E+\infty$ & $1.000 E+00$ & $1.000 E+00$ & $1.000 E+00$ & $1.000 E+\infty$ \\
\hline 1.8 & : & $1.000 E+00$ & $1.000 E+00$ & $1.000 E+00$ & $1.000 E+00$ & $1.000 E+\infty 0$ & $1.000 E+00$ & $1.000 E+00$ & $1.000 E+00$ & $1.000 E+00$ & $1.000 E+00$ \\
\hline 1.6 & : & 1. $000 E+00$ & $1.000 E+00$ & $1.000 E+00$ & $1.000 E+\infty$ & $1.000 E+00$ & $1.000 E+00$ & $1.000 E+00$ & $1.000 E+00$ & $1.000 E+00$ & 1. $000 E+00$ \\
\hline-1.4 & : & $1.000 E+00$ & $1.000 E+00$ & $1.000 E+00$ & $1.000 E+00$ & $1.000 E+\infty 0$ & $1.000 E+00$ & 1. $000 E+\infty 0$ & $1.000 E+00$ & $1.000 E+\infty$ & $1.000 E+00$ \\
\hline-1.2 & : & $1.000 E+00$ & $1.000 E+00$ & $1.000 E+\infty 0$ & $1.0005+\infty 0$ & 1. $0005+\infty$ & $1.000 E+00$ & $1.000 E+\infty 0$ & $1.000 E+\infty$ & $1.0005+00$ & $1.000 E+00$ \\
\hline-1 & $:$ & $1.000 E+00$ & $1.000 E+\infty 0$ & $1.000 E+\infty$ & 1. $000 E+00$ & $1.000 E+00$ & 1. $000 E+00$ & $1.000 E+00$ & $1.000 E+00$ & $1.000=+\infty$ & $1.000 E+\infty 0$ \\
\hline. .8 & $:$ & $1.000 E+00$ & 1. $000 E+00$ & $1.000 E+\infty 0$ & $1.000 E+00$ & $1.000 E+\infty 0$ & $1.000 \varepsilon+\infty 0$ & $1.000 E+00$ & $1.000 E+00$ & $1.000 E+\infty$ & $1.000 E+\infty$ \\
\hline .6 & : & $1.000 E+00$ & $1.000 E$ & & & 1.000 & 1.000 & $1.000 E$ & $1.000 E+00$ & $1.000 E+\infty$ & 1. $000 E+00$ \\
\hline-.4 & : & $1.000 E+\infty$ & $1.000 E+\infty 0$ & $1.000 E+00$ & 1.000E+00 & $1.000 E+00$ & $1.000 E+\infty 0$ & $1.000 E+\infty 0$ & $1.000 E+00$ & $1.000 E+\infty 00$ & $1.000 E+\infty 0$ \\
\hline-.2 & : & $1.000 E+00$ & $1.000 E+00$ & $1.000 E+00$ & 1. $000 E+00$ & $1.000 E+\infty 0$ & $1.000 E+00$ & $1.000 E+00$ & $1.000 E+00$ & 1.000E+00 & 1.000E+00 \\
\hline 0 & $:$ & $1.000 E+00$ & 1. $000 E+00$ & $1.000 E+\infty 0$ & $1.000 E+\infty 00$ & 1.000EPOC & $1.000 E+\infty$ & $1.000 E+\infty$ & $1.000 E+\infty$ & $1.000 E+\infty 0$ & \\
\hline .2 & $:$ & $1.091 E+\infty 0$ & $1.210 E+00$ & $1.360 E+00$ & $1.541 E+00$ & 1. $757 E+\infty 0$ & $2.009 \div+\infty 0$ & $2.3055+00$ & $2.642 E+\infty 0$ & $3.035 \leq+00$ & \\
\hline .4 & $:$ & $1.1996+00$ & $1.487 E$ & $1.890 E+00$ & 2. $441 E+00$ & & $4.174 \varepsilon+\infty$ & $5.489 \varepsilon+\infty$ & $7.231 E+\infty 0$ & $9.535 E+00$ & \\
\hline .6 & : & $1.327 E+\infty$ & $1.854 E+\infty$ & $2.680 E+\infty 0$ & $3.9585+\infty 0$ & $5.916 E+00$ & $8.9035+00$ & $1.344 E+01$ & $2.034 E+01$ & 3.000E+01 & \\
\hline .8 & : & $1.482 E+00$ & $2.343 E+\infty 0$ & $3.867 \mathrm{E}+00$ & 6.5436 & $1.123 E+01$ & $1.940 E+01$ & 3. 364E+01 & $5.846 E+01$ & $1.01 \pi+02$ & \\
\hline 1 & $:$ & $1.667 E+\infty 0$ & $3.000 E+00$ & $5.66 \pi+00$ & 1. 100E+01 & $2.16 \pi+01$ & 4. $300 E+01$ & $8.567 E+01$ & $1.710 E+02$ & $3.417 E+02$ & \\
\hline 1.2 & : & $1.88 \%+\infty$ & 3. $885 E+00$ & $8.415 E+00$ & $1.875 E+01$ & $4.243 E+01$ & $9.672 x+01$ & $2.213 E+02$ & $5.075 E+02$ & $1.165 E+03$ & \\
\hline 1.4 & : & $2.156 E+00$ & $5.084 E+00$ & $1.264 E+01$ & $3.236 E+01$ & $8.40 \% £+01$ & $2.202 E+02$ & $5.789 E+02$ & $1.525 E+03$ & $4.020 E+03$ & \\
\hline 1.6 & : & $2.477 E+00$ & $6.714 E+\infty 0$ & $1.91 \% E+01$ & $5.642 E+01$ & $1.684 E+02$ & $5.066 E+02$ & $1.530 E+03$ & $4.622 \varepsilon+03$ & $1.402 \sum+04$ & \\
\hline 1.8 & : & $2.865 E+00$ & $8.943 E+00$ & $2.938 E+01$ & $9.926 E+01$ & 3. $404 E+02$ & $1.176 E+03$ & $4.080 E+03$ & $1.418 E+04$ & $4.932 E+04$ & \\
\hline 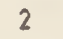 & : & $3.333 E+00$ & $1.200 E+01$ & 4.533E+01 & $1.760 E+02$ & $6.933 E+02$ & $2.752 E+03$ & $1.097 E+04$ & $4.378 E+04$ & 1.749E+05 & \\
\hline
\end{tabular}


$B 2(r$, aus $)$

\begin{tabular}{|c|c|c|c|c|c|c|c|c|c|c|c|}
\hline 11 & $r$ & .0 & .0003 & .001 & .003 & .01 & .03 & .1 & .3 & .5 & .7 \\
\hline-2 & 8 & $E-01$ & $E-01$ & -01 & 0.001502 & 8 & 8 & (1) & 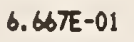 & & \\
\hline 1.8 & 8 & $1.112 \leq-01$ & $1.365 E-01$ & $1.76 x=01$ & 2. $195 E-01$ & $3.793 E-01$ & 3. $47 \% E-01$ & $4.431 E-01$ & $5.566 E-01$ & -01 & \\
\hline 1.6 & $:$ & $1.874 E-02$ & $.900<-02$ & 4. $700 E-02$ & $7.306 E-02$ & $.185 E-01$ & $1.8365-01$ & $2.979 E-01$ & $4.6935-01$ & 5. $901 E-01$ & $.013 E-01$ \\
\hline .4 & : & $x-03$ & $196 E-03$ & $1.276 E-02$ & -02 & $E-02$ & $c \varepsilon-02$ & 01 & 01 & & 61E-01 \\
\hline 1.2 & : & $5.586 E-04$ & $345 E-03$ & $3.52 x x-03$ & 1. $49 \%=-03$ & $.25 x-02$ & $362 x-02$ & $1.410 E-01$ & 1. 4HE-01 & $.273 E-01$ & $.05 x-01$ \\
\hline & : & $1.000<-04$ & $.000 E-04$ & $O E-\infty 3$ & $000 E-03$ & $.000<-02$ & $000 E-02$ & $1.000 E-01$ & 1 & 1 & OOE-01 \\
\hline 8 & 8 & $1.86 z-05$ & $956 E-05$ & TE-OA & $101 E-03$ & $1.6625-03$ & $73 x E-02$ & $7.271 E-02$ & 01 & & $16 E-01$ \\
\hline- & $:$ & $3.687 E-\infty 6$ & $715 E-05$ & $.231 E-05$ & $200 E-04$ & $28:=-03$ & $04 \pi-02$ & $5.430 E-02$ & $2.351 E-01$ & $4.51 \pi-01$ & $.800 E-01$ \\
\hline .4 & : & & $E=-\infty 6$ & & & & & & & & \\
\hline-.2 & 8 & $\varepsilon-07$ & $x=-06$ & $1.260 E-05$ & $8.6065-05$ & $1 E-04$ & $E-\infty$ & $3.340 E-02$ & 1 & 1 & . 546E-01 \\
\hline 0 & : & $26 E-\infty 8$ & .240E-07 & $6.065 E-06$ & $4.745 E-05$ & AE-OA & $250 E-03$ & $2.742 E-02$ & 1. & 01 & $01 E-01$ \\
\hline .2 & : & $06 E-\infty 8$ & $.397 t-07$ & $.994 E-06$ & $048 E-05$ & 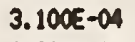 & $.494 \varepsilon-03$ & 2 & & & $E-01$ \\
\hline .4 & $:$ & $-\infty$ & 07 & $-\infty 6$ & $228 E-\infty 5$ & & -03 & & & & $E-01$ \\
\hline .6 & : & $-\infty$ & $800 \leq-07$ & 06 & 1. $7208-\infty$ & & $.70 \leq \varepsilon-03$ & $1.773 E$ & .308 & -01 & $3.943 E-01$ \\
\hline .8 & $:$ & $-\infty 8$ & $529 E-07$ & 06 & $1.524 E-05$ & $5-$ & $.493 \varepsilon-03$ & $1.5935-02$ & 1 & -01 & . $789 E-01$ \\
\hline & $:$ & $1.500 E-08$ & . 350E-07 & $1.500 E-06$ & $1.349-05$ & $1.495 x-04$ & $.337 t=03$ & $1.450 E-02$ & 1.2 & $3.125 z-01$ & $5.635 E-01$ \\
\hline 1.2 & : & & & & & 30. & & & & & $x E-01$ \\
\hline 1.4 & 8 & $1.245 E-08$ & $120 E-07$ & $1.245 E-\infty 6$ & $1.120=-05$ & $1.244 E-04$ & $1.118<-03$ & $1.233 E-02$ & 1.074 & $2.85 \% E-01$ & 5. $333 E-01$ \\
\hline & 8 & $1.152 \leq-\infty$ & $.037 E-07$ & $1.152 E-06$ & (e) & - & $1.0365-03$ & $1.14 \pi-02$ & & -01 & $.186 E-01$ \\
\hline 1.8 & : & $1.0725-08$ & $9.645 E-08$ & $1.072 E-06$ & $9.645 E-06$ & $1.0725-04$ & $9.642 E-04$ & $1.070 E-02$ & 9.541 & $2.615 E-01$ & 5. $042 x-01$ \\
\hline 2 & 8 & $1.000 E-08$ & $9.000 E-08$ & $1.000 E-06$ & $9.000 E-06$ & $1.000 E-04$ & $9.000 E-04$ & $1.000 E-02$ & $9.000 E-02$ & $2.500 E-01$ & 4. $900 E-01$ \\
\hline
\end{tabular}




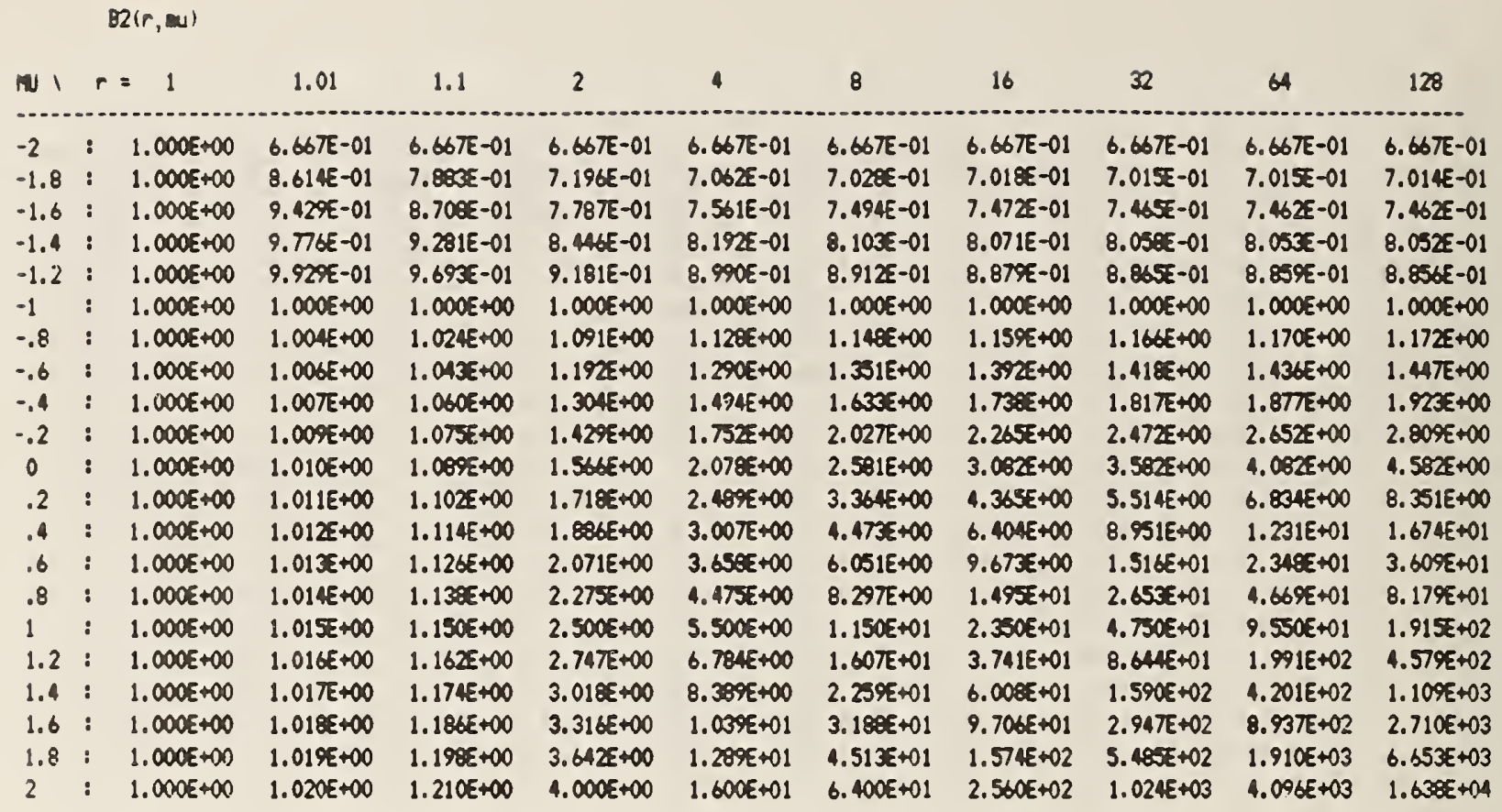

\begin{tabular}{|c|c|c|c|c|c|c|c|}
\hline mi 1 & $r=$ & 256 & 512 & 1024 & 2048 & 4096 & INF \\
\hline-2 & : & $6.667 E-01$ & $6.667 E-01$ & $6.667 E-01$ & $6.667 E=01$ & $6.66 \pi E-01$ & $6.667 E-01$ \\
\hline-1.8 & : & $7.014 E-01$ & $7.014 E-01$ & $7.014 E-01$ & $7.014 E-01$ & $7.014 E-01$ & $7.014 E-01$ \\
\hline-1.6 & : & $7.461 E-01$ & $7.461 E-01$ & $7.461 E-01$ & $7.461 E-01$ & $7.461 E-01$ & $7.461 E-01$ \\
\hline-1.4 & : & $6.051 E-01$ & $8.051 E-01$ & $8.051 E-01$ & $8.051 E-01$ & $8.051 E-01$ & $8.051 E-01$ \\
\hline-1.2 & : & $3.8555-01$ & $8.854 E-01$ & $8.854 E-01$ & $8.854 E-01$ & $8.854 E-01$ & $8.854 E-01$ \\
\hline-1 & : & 1. $000 E+\infty 0$ & $1.000 E+100$ & $1.000 E+00$ & 1.000E +00 & $1.000 E+\infty$ & $1.000=+00$ \\
\hline-.8 & : & $1.173 E+(x)$ & $1.174 E+00$ & $1.174 E+\infty 0$ & $1.174 E+\infty 0$ & $1.174 E+\infty$ & $1.175 E+\infty$ \\
\hline-.6 & : & $1.455 E+\infty 0$ & $1.460 E+100$ & $1.463 E+\infty 0$ & $1.465 E+00$ & $1.46 \pi+\infty 00$ & $1.470 E+00$ \\
\hline-.4 & : & $1.95 \pi+\infty 0$ & $1.983 E+00$ & $2.003 E+00$ & $2.018 \varepsilon+\infty 0$ & $2.029 E+100$ & $2.065 E+\infty 0$ \\
\hline-.2 & $:$ & $2.9455+00$ & $3.064 E+00$ & $3.16 \pi=00$ & $3.25 \pi E+\infty 0$ & $3.3365+100$ & $3.86 .35+00$ \\
\hline 0 & : & $5.002 E+100$ & $5.582 E+00$ & $6.082 E+00$ & $6.582 E+00$ & $7.082 E+100$ & \\
\hline.$\hat{8}$ & ; & 1.009E+01 & $1.2096+01$ & $1.43 \%+01$ & $1.703 E+01$ & $2.006 E+01$ & \\
\hline .4 & : & $2.259 E+01$ & $3.031 E+01$ & $4.050 E+01$ & $5.394 E+01$ & $7.167 \mathrm{E}+01$ & \\
\hline.$\dot{s}$ & : & $5.521 E+01$ & $8.418 E+01$ & $1.281 E+02$ & $1.947 E+02$ & $2.955 E+02$ & \\
\hline .8 & : & $1.429 E+02$ & $2.493 E+02$ & $4.346 E+02$ & $7.571 E+02$ & $1.319 E+03$ & \\
\hline 1 & : & $3.835 E+02$ & $7.675 E+02$ & $1.5365+03$ & $3.0725+03$ & $6.144 E+03$ & \\
\hline 1.2 & : & $1.0525+03$ & $2.418 E+03$ & $5.5565+03$ & $1.2 \pi \pi+04$ & $2.933 E+04$ & \\
\hline 1.4 & $:$ & $2.928[1+13$ & $7.72 \pi+03$ & $2.03 \%+04$ & $5.382 E+04$ & $1.420 E+05$ & \\
\hline 1.6 & : & $8.215 E+03$ & $2.490 E+04$ & $7.5495+04$ & $2.280 E+05$ & $6.937 \mathrm{E}+05$ & \\
\hline 1.8 & $:$ & $2.31 \pi+\infty$ & $8.067 E+04$ & $2.809 \%+05$ & $9.782 E+05$ & 3. $406 E+06$ & \\
\hline 2 & : & $6.554 E+04$ & $2.621 E+05$ & $1.049 E+06$ & 4.194E +06 & $1.678 E+07$ & \\
\hline
\end{tabular}


$23(2, \mu, r, w)$ for $r \bullet .01$

\begin{tabular}{|c|c|c|c|c|c|c|c|c|c|c|c|c|}
\hline & & 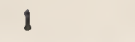 & 2 & $\checkmark$ & & & & 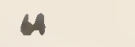 & 88 & 256 & 12 & 24 \\
\hline & 8 & $E+\infty$ & $+\infty$ & $E+\infty$ & $:+\infty$ & 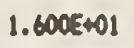 & 1 & 1 & 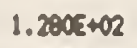 & & & \\
\hline & . & $1.000<+00$ & & & & & & & & & & \\
\hline & 8 & & & & & $\div 02$ & & & 100 & $20 x+\infty$ & & $: 8$ \\
\hline & 1 & & & & & & & & & & & \\
\hline & 8 & $1.000 E+\infty 0$ & $\infty$ & $E+\infty$ & 3. $043 E+01$ & & & $+\infty 0$ & $25 x+\infty$ & & & \\
\hline & : & & & & ol & & 02 & 3 & 10 & 03 & $\boldsymbol{\infty}$ & 5 \\
\hline & 8 & & 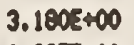 & & & & & & & & & \\
\hline & 8 & & & & 4.8 & & 02 & 2.3 & 103 & & 03 & 10 \\
\hline & 1 & & & & & & & & & & & \\
\hline & : & & & & & & & & & & & +0 \\
\hline & 8 & & & & & & & & & & & \\
\hline .2 & 1 & & & & & & & & & & & +0 \\
\hline .4 & 1 & & 00 & & & & & & 02 & 02 & & $=40$ \\
\hline 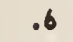 & : & & 2.5 & & & 01 & & & 02 & & & +0 \\
\hline & 1 & & & & & & & & & & & $E$ \\
\hline & 1 & & 1.9 & & $:+\infty$ & 1.4 & & & & & & $\varepsilon+C$ \\
\hline & & & 1.73 & & $=+\infty$ & $8 . T$ & & & +01 & 2.85 & 01 & $2.933 E+$ \\
\hline & & & & & & & & & & & & $.272 E+$ \\
\hline & & 1.000 & & 1.73 & $2.260 E+\infty 0$ & 2.98 & 3.82 & 4.67 & $+\infty$ & 5.40 & $+\infty$ & $5.473 E \hbar$ \\
\hline & $:$ & & & & & & & & & & $2.340 E+\infty 0$ & 2.3 \\
\hline & 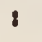 & 1.000 & $1.000 \leq+\infty 0$ & $1.0005+00$ & $1.000 E \div+00$ & $1.000 E+00$ & $0<+\infty 0$ & $1.000 \varepsilon+\infty$ & $\varepsilon+\infty$ & $1.000 E+00$ & $1.000 E+\infty$ & 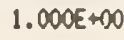 \\
\hline
\end{tabular}

$03(2, n, r$, wu $)$ for $r=.03$

\begin{tabular}{|c|c|c|c|c|c|c|c|c|c|c|c|c|}
\hline 4 & $H=$ & d & 2 & 4 & 8 & 16 & 32 & 64 & 128 & 256 & 512 & 1024 \\
\hline .8 & : & 1. $000 E+\infty 0$ & $216 E+100$ & $8665+00$ & $.865 E+01$ & $1385+01$ & $81 \approx \boldsymbol{z}+02$ & 4. $800 E+02$ & $6.534 E+02$ & $8.075 E+02$ & $.64 \pi t+02$ & $143 E+03$ \\
\hline 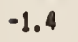 & $:$ & 1.000E+00 & $2.623 E+00$ & $8.81 \pi+00$ & $3.378 E+01$ & $1.411 E+02$ & $5.073 E+02$ & $8.205 E+02$ & $1.022 E+03$ & $1.147 E+03$ & $1.22 \% E+03$ & $1.283 E+03$ \\
\hline .2 & : & 1.000E+00 & $2.816 E+00$ & $1.000 E+01$ & $3.910 E+01$ & $1.60 \pi+02$ & $5.3865+02$ & $8.548 E+02$ & $1.02 \pi+03$ & $1.125 x+03$ & $1.180 \varepsilon+03$ & $1.21 \approx E+03$ \\
\hline & : & $1.000 \leqslant+\infty 0$ & $3.000 E+\infty 0$ & $1.100 E+01$ & +01 & $1.710 E+02$ & $5.328 \varepsilon+02$ & 8.22 & $9.66 \pi+02$ & $039 E+03$ & $1.075 E+03$ & $E+03$ \\
\hline & $:$ & $1.000 \leqslant+\infty$ & $3.3565+\infty 0$ & $1.213<+01$ & $4.331 E+01$ & $1.4555+02$ & $3.618 E+02$ & 5.12 & $5.743 E+02$ & $5.974 E+02$ & 6.057 & 6.06 \\
\hline 2 & : & $1.000 E+\infty$ & $3.3395+\infty 0$ & $1.157 \mathrm{E}+01$ & $3.896 E+01$ & $1.214 E+02$ & $2.79 \pi+02$ & 3.85 & $4.262 E+02$ & 4. $405 E+02$ & $4.45 x+02$ & $4.468 E+02$ \\
\hline 0 & : & $1,000 E+\infty 0$ & 3. $2295+\infty$ & $1.052 E+01$ & $3.290 E+01$ & $9.462 x+01$ & $2.019 E+02$ & $2.702 E+02$ & $2.957 E+02$ & 3. $040 E+02$ & $3.065 E+02$ & $3.074 E+122$ \\
\hline .2 & : & $1.000 \leqslant+\infty$ & $3.036 E+\infty 0$ & $9.146 E+\infty$ & $2.635 \mathrm{E}+01$ & $6.9125+01$ & $1.366 E+02$ & 1.77 & $1.924 E+02$ & $1.970 \Sigma+02$ & 1.983 & 1.98 \\
\hline .4 & : & $1.000 E+\infty 0$ & $2.787 E+\infty$ & $7.6405+00$ & $2.0025+01$ & $4.774 E+01$ & 8. $737 E+01$ & 1.10 & $1.184 E+02$ & $1.20 \%+02$ & $1.215 x+02$ & $E+02$ \\
\hline & $:$ & $1.000 \leqslant+\infty 0$ & $1.508 \varepsilon+\infty 0$ & $2.25 \%++\infty$ & $3.322 E++00$ & $4.658 E+00$ & $5.829 E+00$ & 6.37 & $6.538 E+\infty 0$ & $6.580 \varepsilon+\infty 0$ & $6.595 E+\infty$ & $6.599 E+\infty 0$ \\
\hline & : & $1.0005+\infty 0$ & $1.316 E+\infty 0$ & $1.72 x x+\infty 0$ & $2.234 E+\infty 0$ & $2.801 E+\infty$ & $3.251 E+\infty 0$ & 3. $450 E+\infty$ & $3.511 E+\infty 0$ & $3.52 \pi E+\infty 0$ & $3.531 E+\infty 0$ & $3.532 \leq+\infty 0$ \\
\hline & $:$ & $1.000 E+\infty 0$ & $1.140 E+\infty$ & $1.3145+\infty 0$ & $1.497 E+00$ & $1.6765+\infty 0$ & $1.805 E+00$ & $\sum+\infty$ & $1.876 E+\infty 0$ & $1.881 E+\infty$ & $1.882 E+\infty$ & $1.88 x+\infty 0$ \\
\hline 2 & : & $1.000 \leqslant+\infty 0$ & $1.000 E+\infty 0$ & $1.000 \leqslant+\infty 0$ & $1.000 \varepsilon+\infty 0$ & $1.000 E+00$ & $1.000 E+\infty 0$ & $1.000 \leqslant+00$ & $1.000 \varepsilon+\infty$ & $1.000 E+00$ & $1.000 E+\infty$ & 1. $000 E+00$ \\
\hline
\end{tabular}


$13(2, M, r$, eu $)$ for $r=.1$

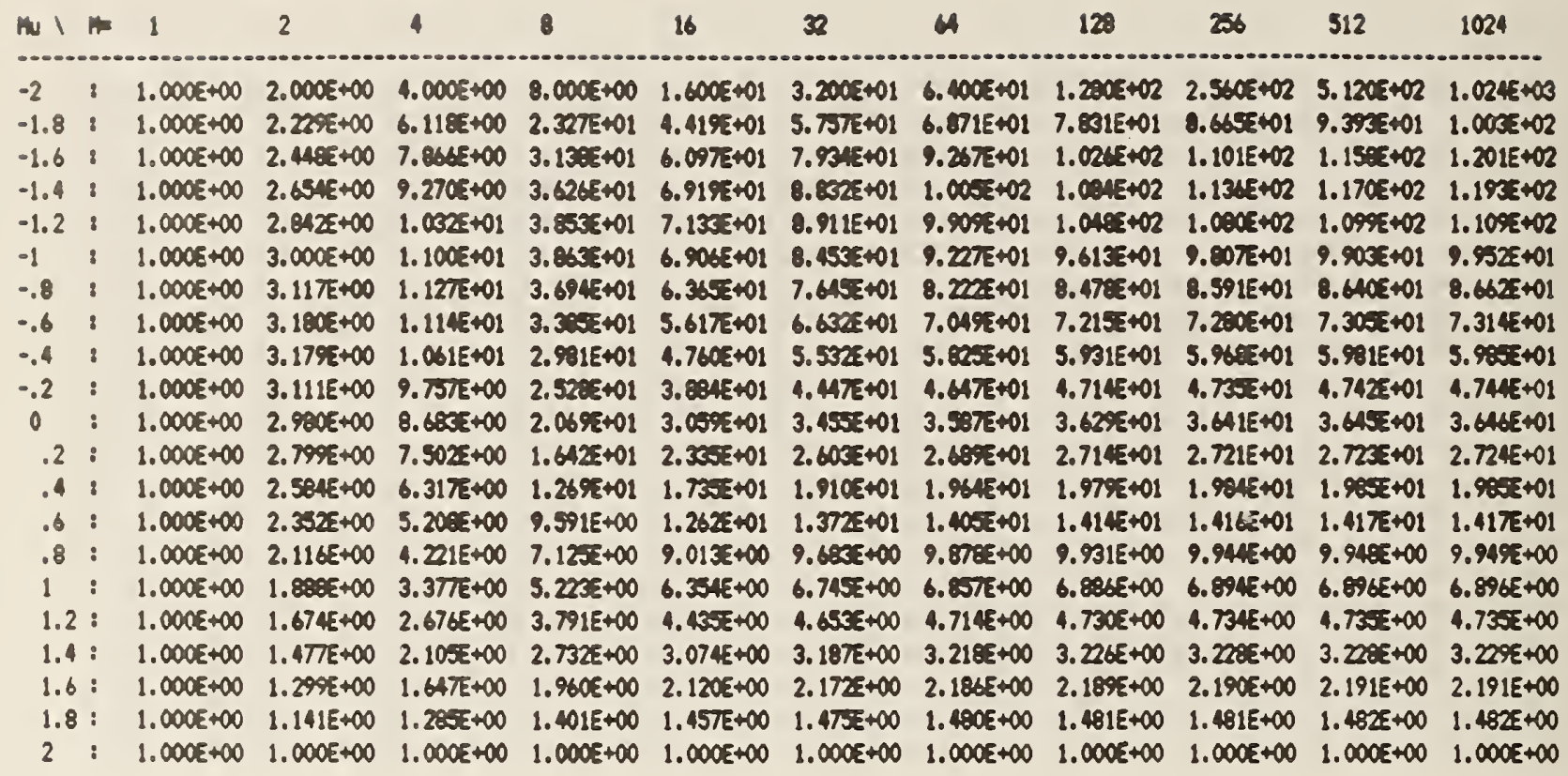

$B 3(2, H, r$, eus $)$ for $r=.3$

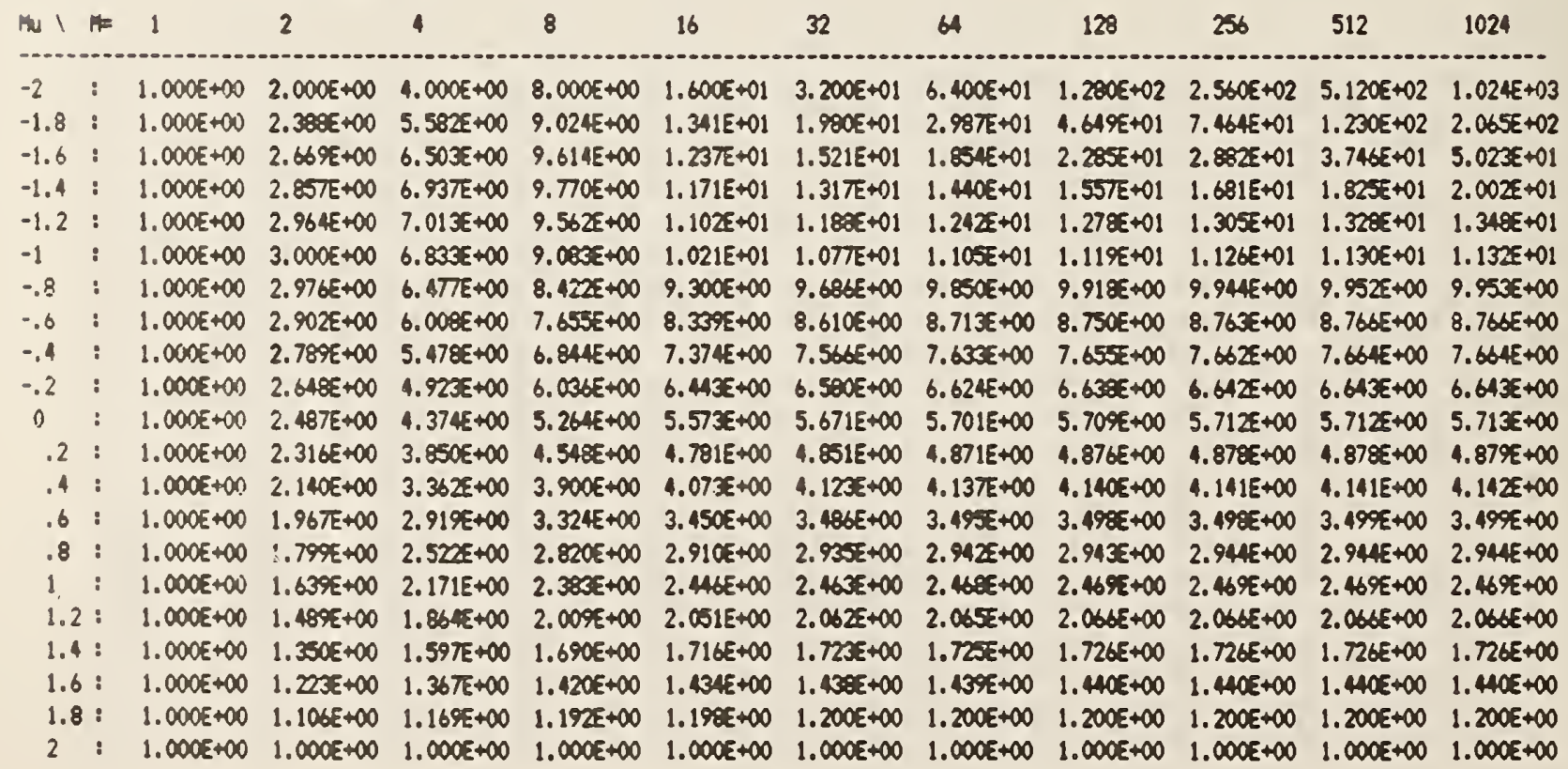




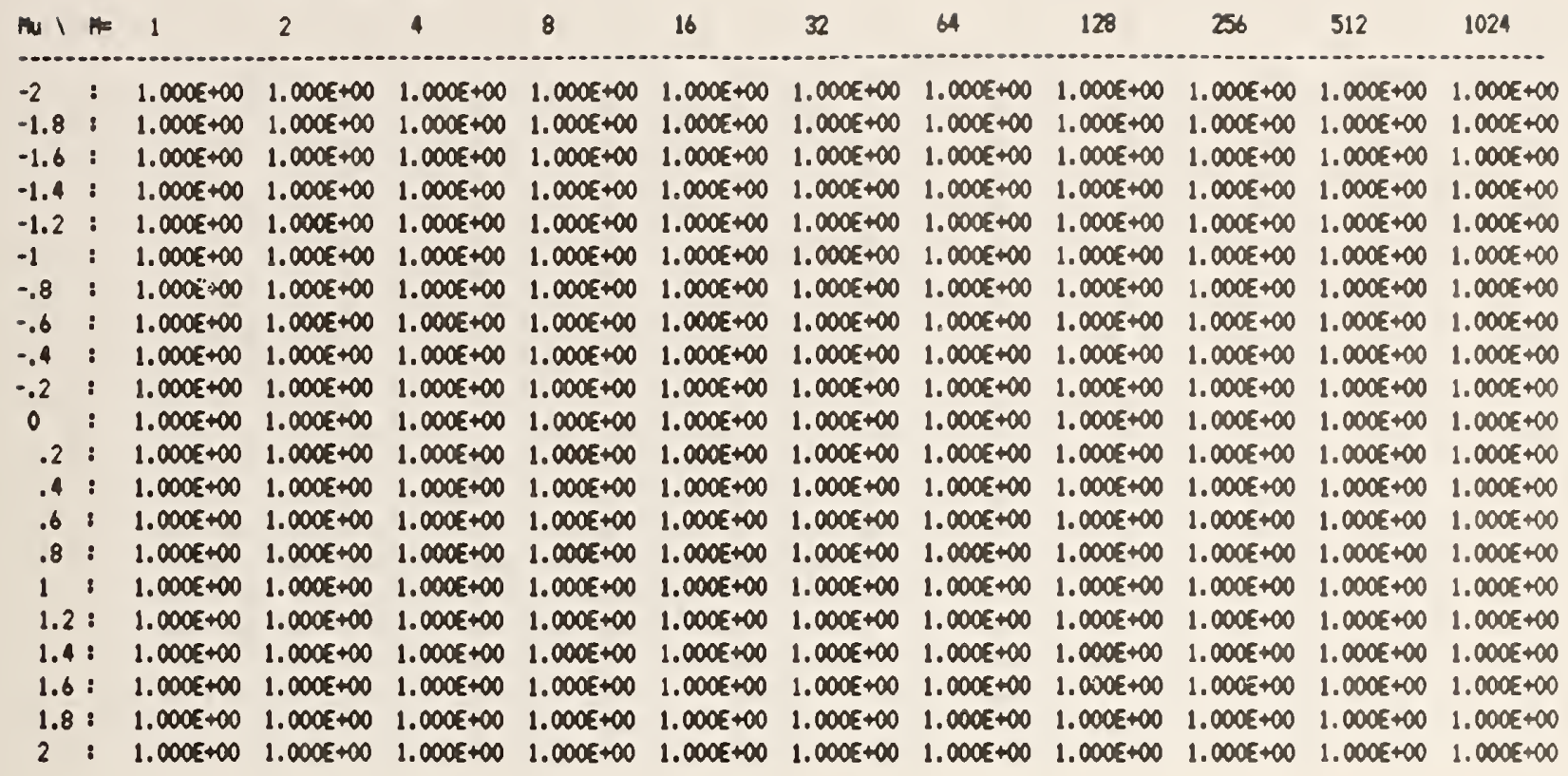

$B 3(2, \mathrm{H}, r$, wu $)$ for $r=1.01$

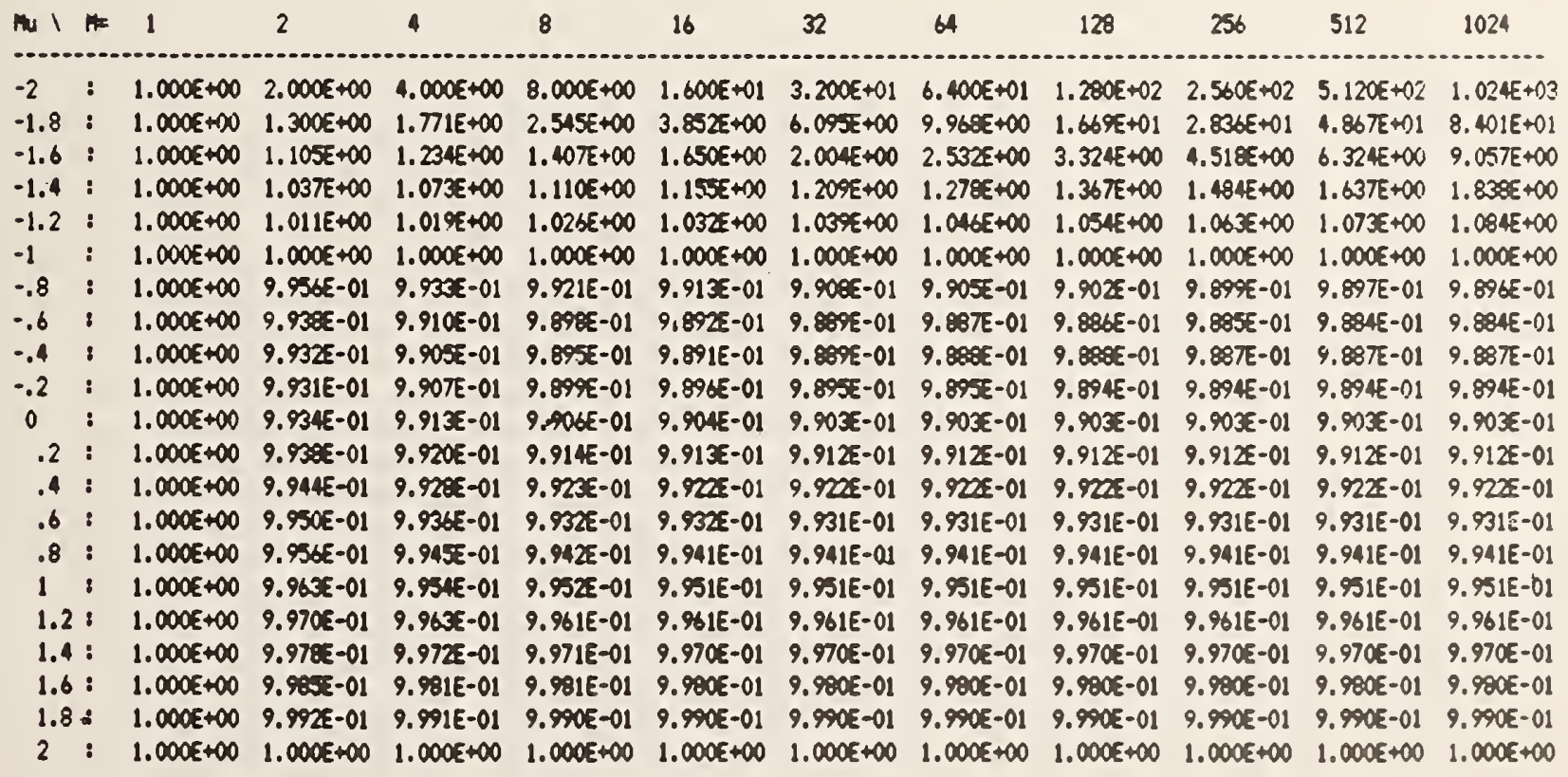


$B 3(2, \mu, r$, au $)$ for $r=1.1$

\begin{tabular}{|c|c|c|c|c|c|c|c|c|c|c|c|c|}
\hline 11 & $=$ & 1 & 2 & 9 & 8 & 16 & 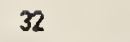 & 64 & 128 & 56 & 512 & \\
\hline .8 & : & $0 E+\infty$ & $494 E+\infty 0$ & $2286 E+\infty 0$ & $604 E+00$ & $+\infty$ & $700 E+00$ & 1 & $795 E+01$ & $808 E+01$ & & \\
\hline .4 & $:$ & $E+\infty$ & & $+\infty$ & $385 E+\infty 0$ & $:+\infty$ & $+\infty$ & & $12 E+\infty$ & $88 \pi+\infty$ & & \\
\hline 1.2 & : & $E+\infty$ & $-\infty$ & $+\infty$ & $.11 \pi+\infty 0$ & $153 E+\infty 0$ & $+\infty$ & 00 & $.282 \mathbb{E}+00$ & $336 E+\infty 0$ & $1.39 \% \varepsilon+00$ & $.470 E+00$ \\
\hline & : & $=+\infty$ & & & $+\infty$ & & 400 & & $:+\infty$ & $D O E+\infty$ & & \\
\hline 8 & : & $E+\infty 0$ & -01 & & $x-01$ & -01 & & & & $E-01$ & & $E-01$ \\
\hline 2 & : & $E+\infty$ & -01 & & & & & & & $E-01$ & & $E-01$ \\
\hline 0 & : & $E+\infty$ & $47 x=01$ & 01 & -01 & 01 & -01 & & $=01$ & $E-01$ & & $E-01$ \\
\hline .2 & . & $0 E+\infty$ & $0 E-01$ & & $=-01$ & & -01 & & -01 & $E-01$ & & $E-01$ \\
\hline .4 & : & $0 E+\infty 0$ & $523 E-01$ & 01 & $x-01$ & -01 & $E-01$ & 01 & $26 E-01$ & $6 E-01$ & -01 & $E-01$ \\
\hline & : & $1.000 E+00$ & $9.798 E-01$ & & $9.739 E-01$ & & $E-01$ & & $9.735 E-01$ & $9.735 E-01$ & $9 . \cdots 35 E-01$ & $.735 E-01$ \\
\hline & 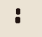 & $x+(x)$ & $9.864 E-01$ & -01 & $.825 E-01$ & $9.823 E-01$ & $822 E-01$ & $9 . \vdots 2$ & $8225=01$ & $9.822 \leq-01$ & $9.822 \leq-01$ & $.8225-01$ \\
\hline 1. & : & $+\infty 10$ & $9.931 E-01$ & & $9.912 E_{0}-01$ & S. : 15-01 & $=-01$ & 9.91 & $E-01$ & $9.911 E-01$ & $9.911 E-01$ & $E-01$ \\
\hline 2 & : & $1.000 E+00$ & $1.000 E+\infty 0$ & 1. NOE- 0 & $1.000 E+n 0$ & $1.000 E+\infty 0$ & $1.000 E+\infty 0$ & 1. $000 E+00$ & $1.000 \sum+00$ & $1.000 E+\infty$ & $1.000 E+00$ & $1.000 \leq+\infty 0$ \\
\hline
\end{tabular}

$B 3(2, M, r, \infty)$ for $r=2$

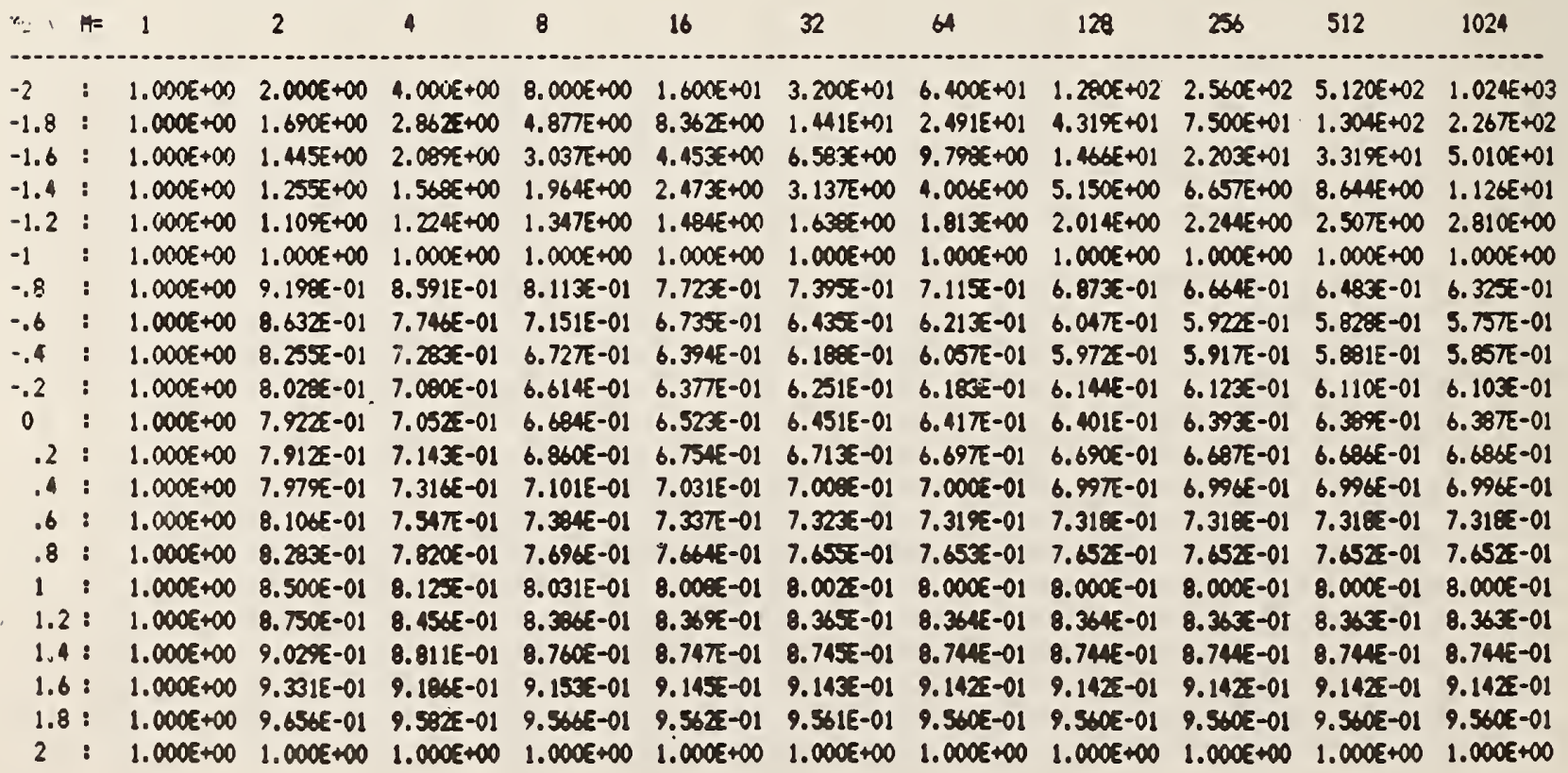


$B(2, n, r, w)$ for $r=4$

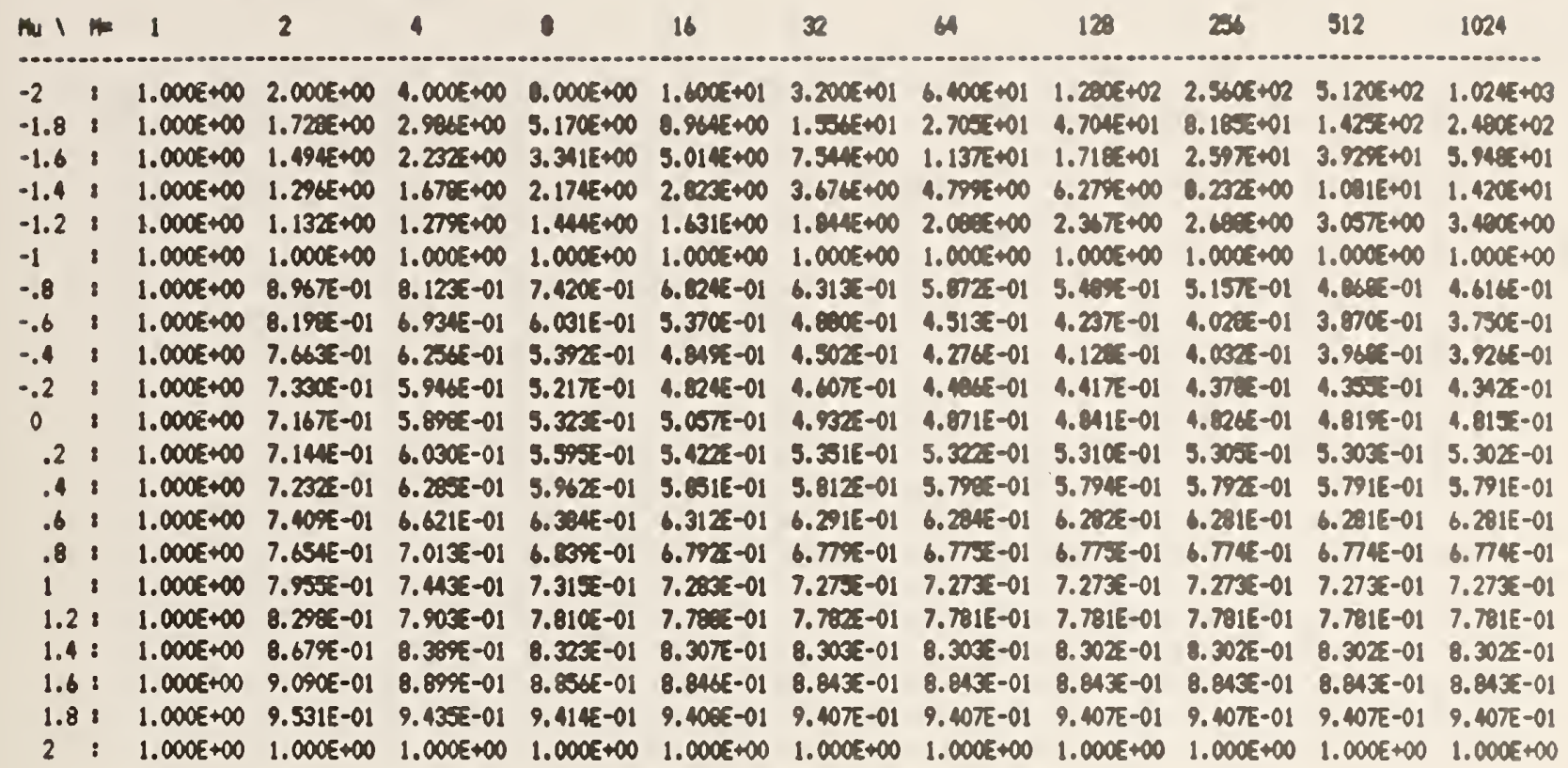

$B 3(2, H, r, \infty u)$ for $r=8$

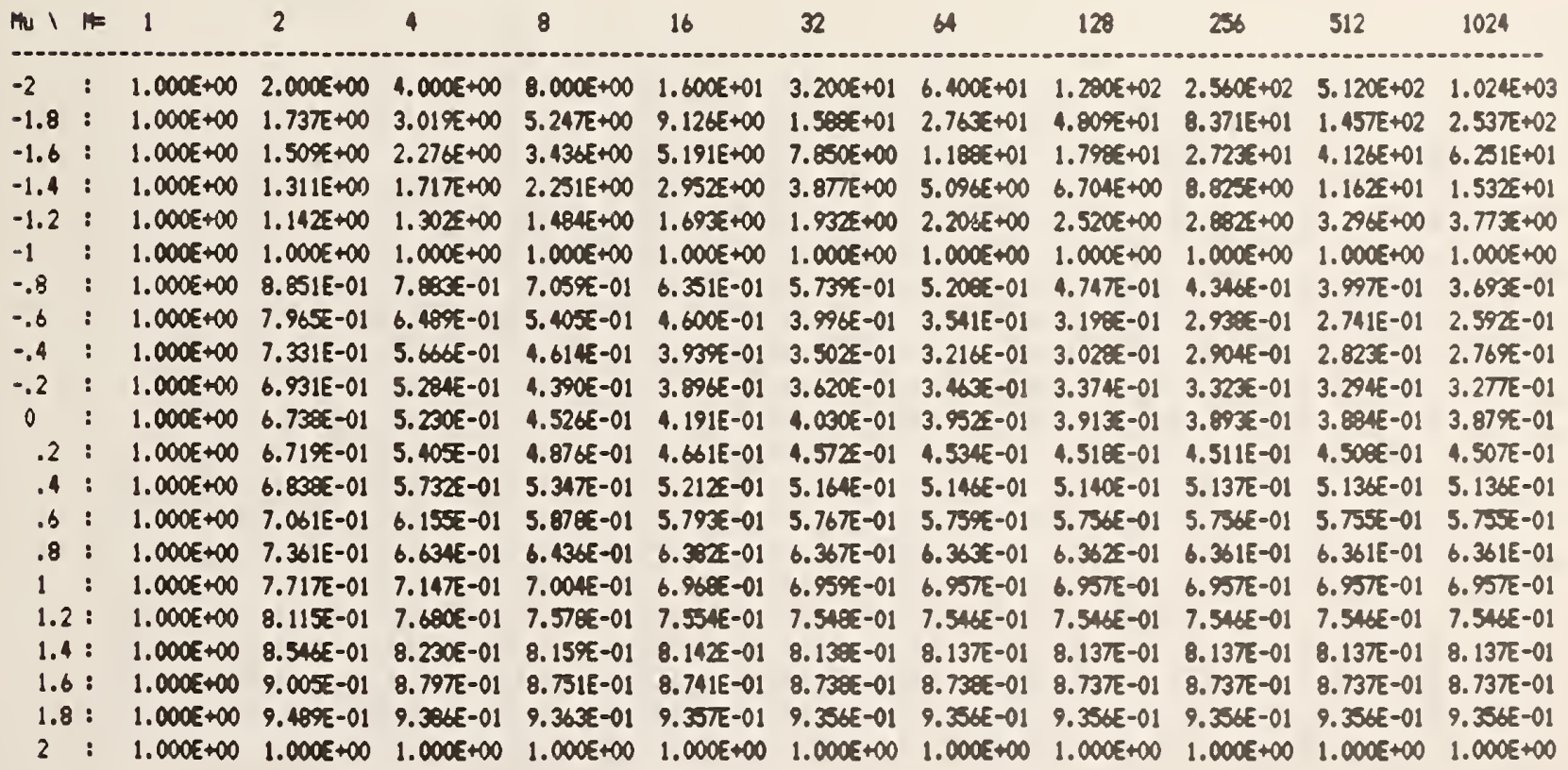


$3(2, n, r, e u)$ for $r=16$

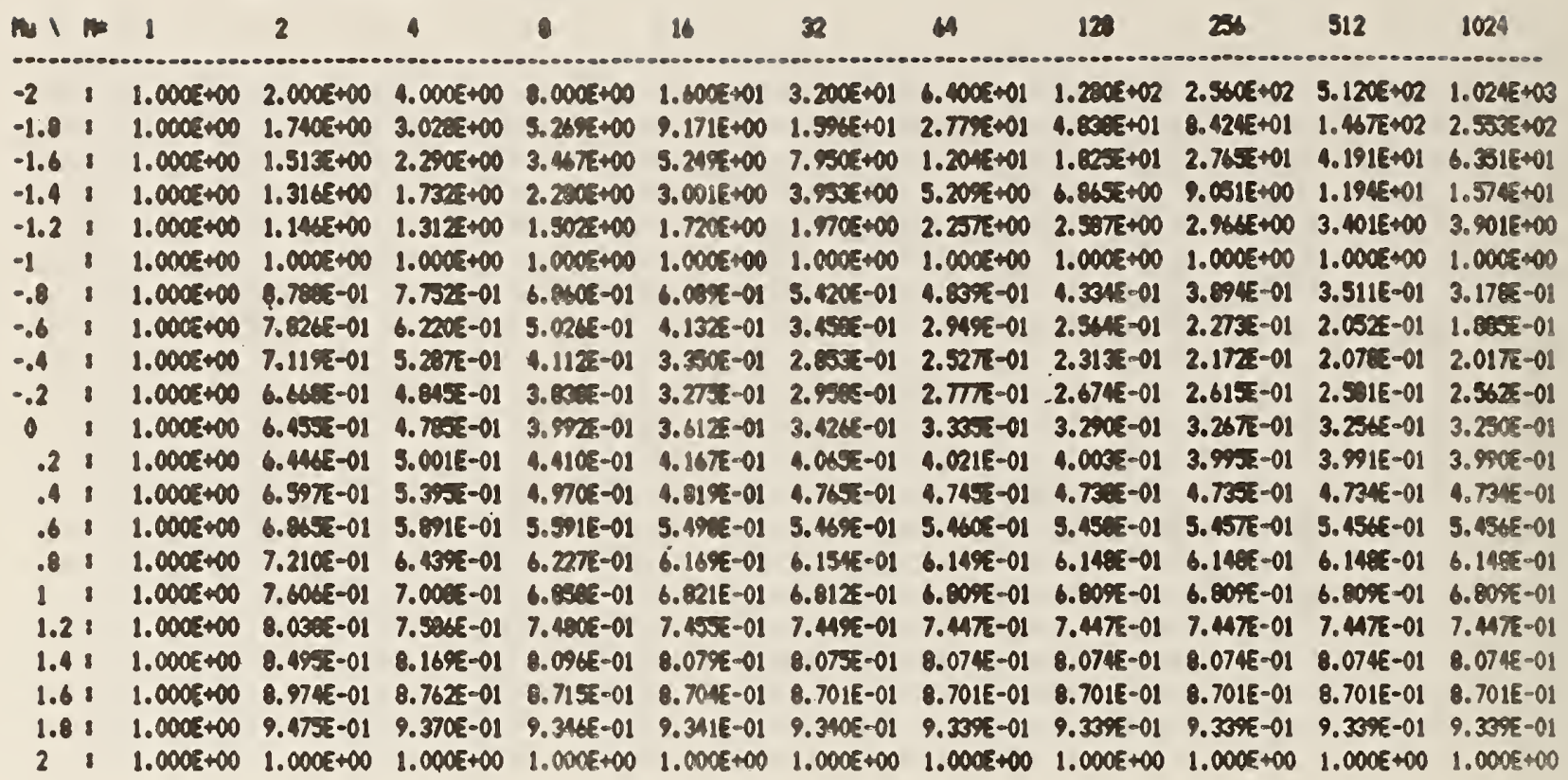

$B(2, A, r$, wu $)$ for $r=32$

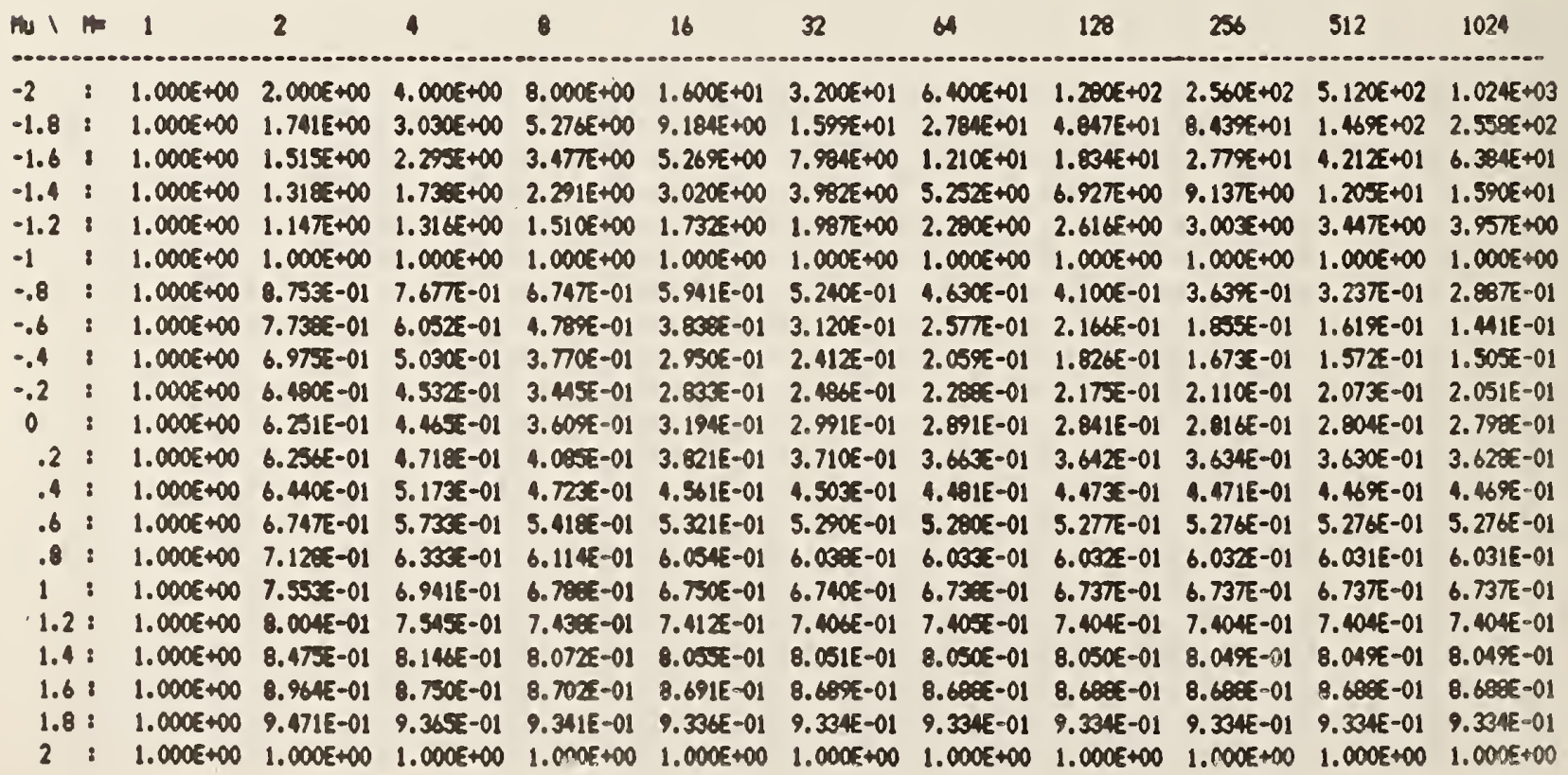


$83(2, H, r, \infty u)$ for $r=64$

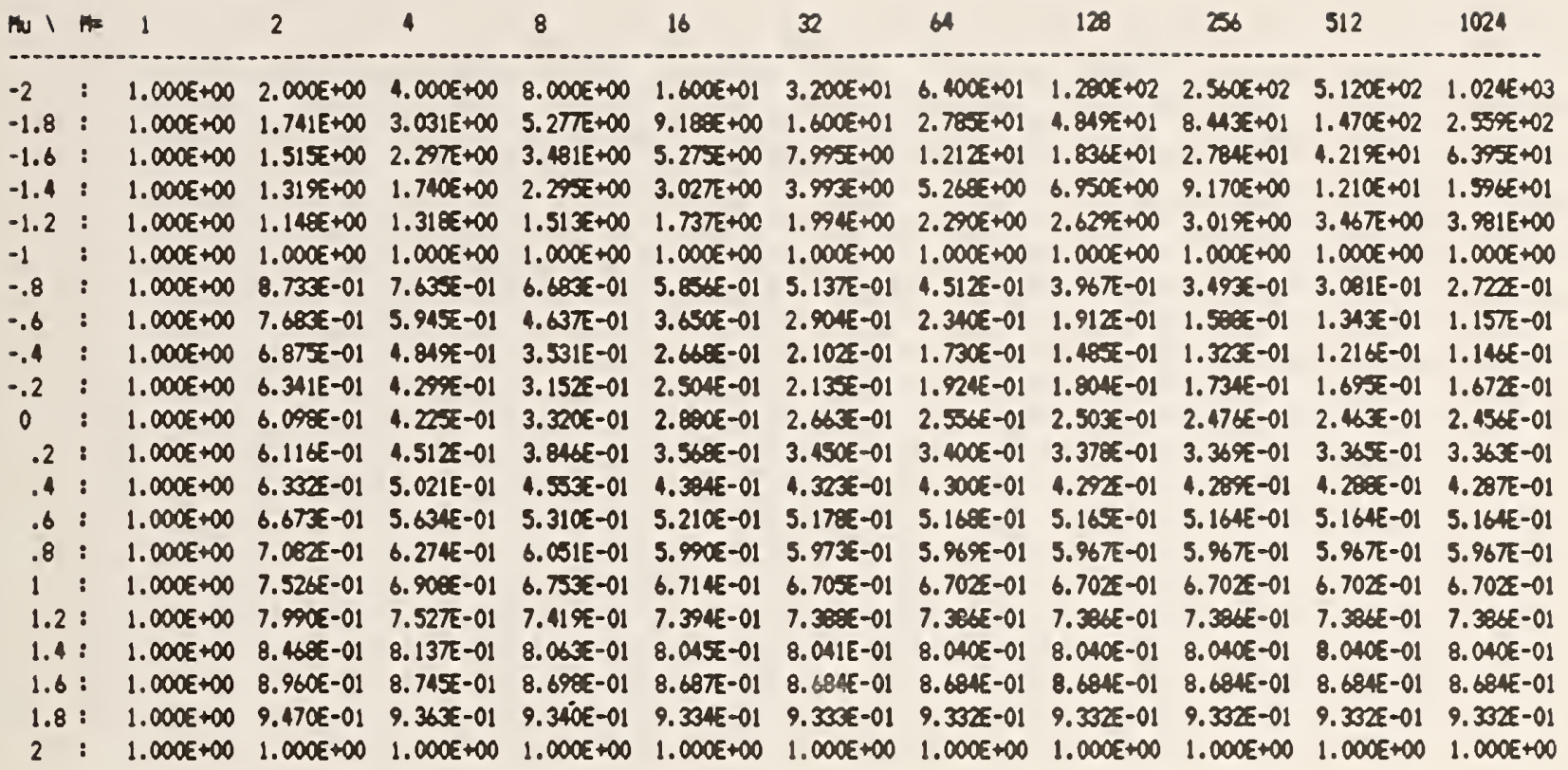

$B 3(2, M, r, w)$ for $r=128$

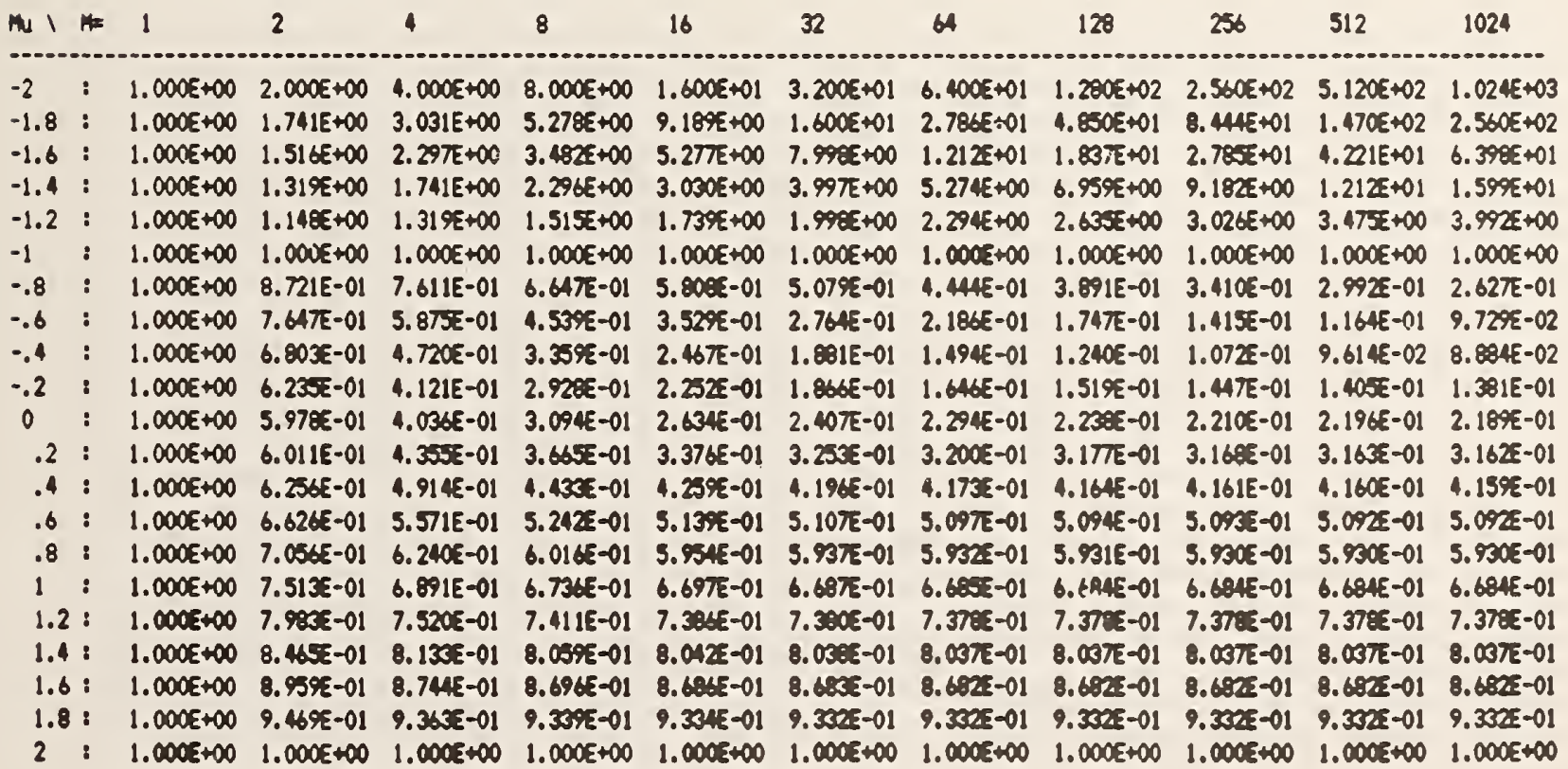


$83(2, \mu, r, a)$ for $r=236$

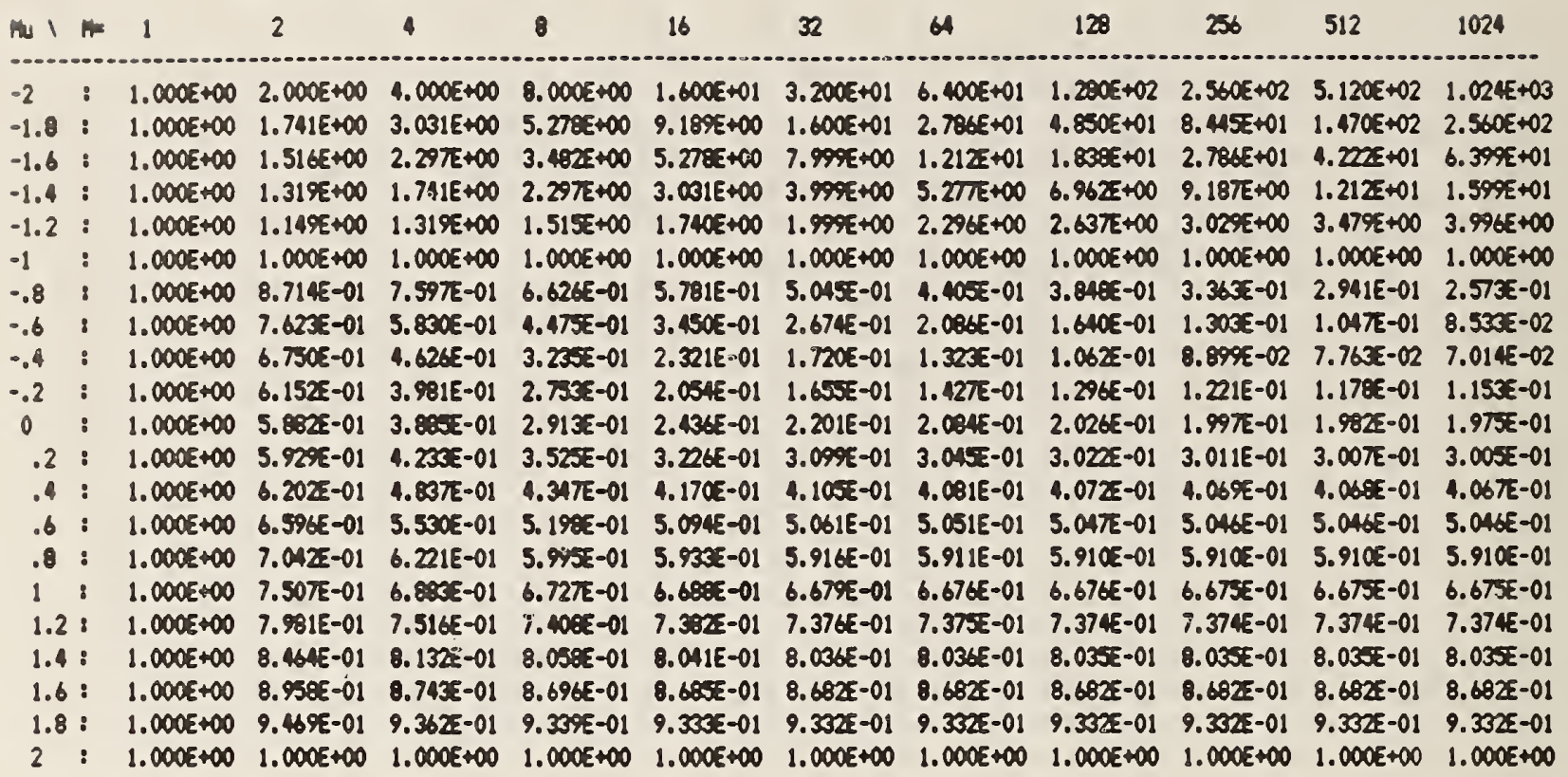

$\$(2, H, r, w)$ for $r=512$

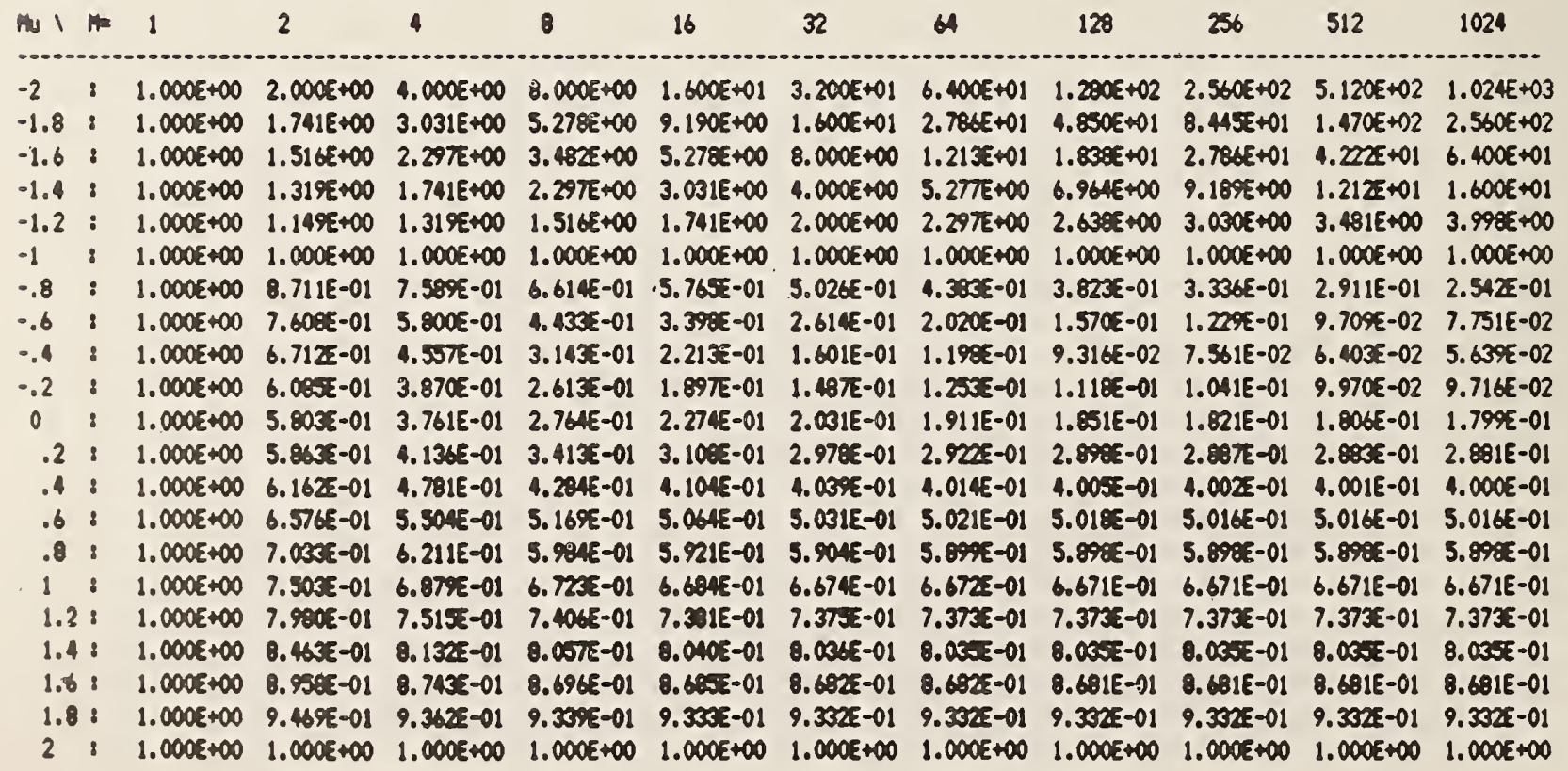




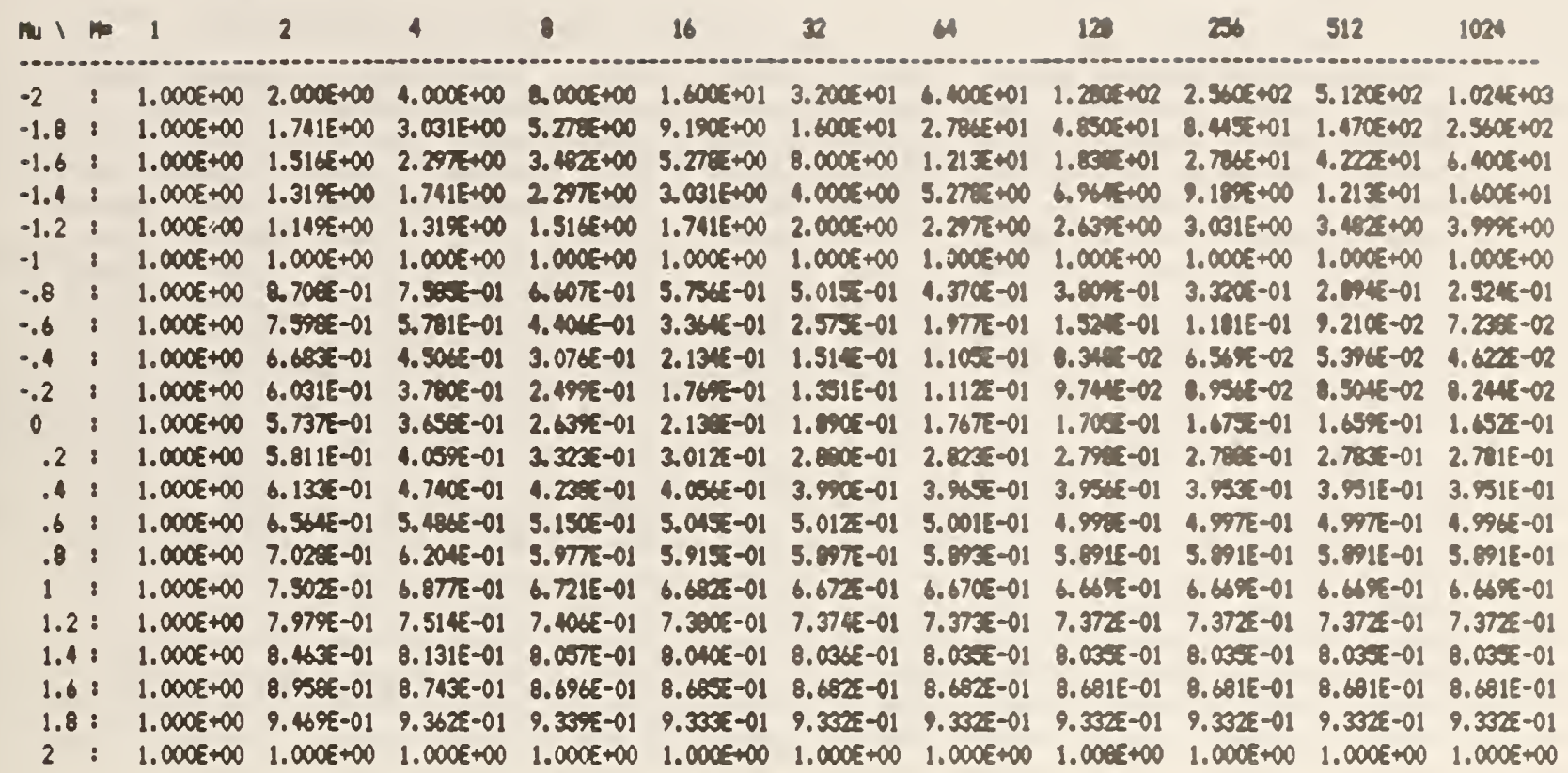

$23(2, n, r$, we for $r=2048$

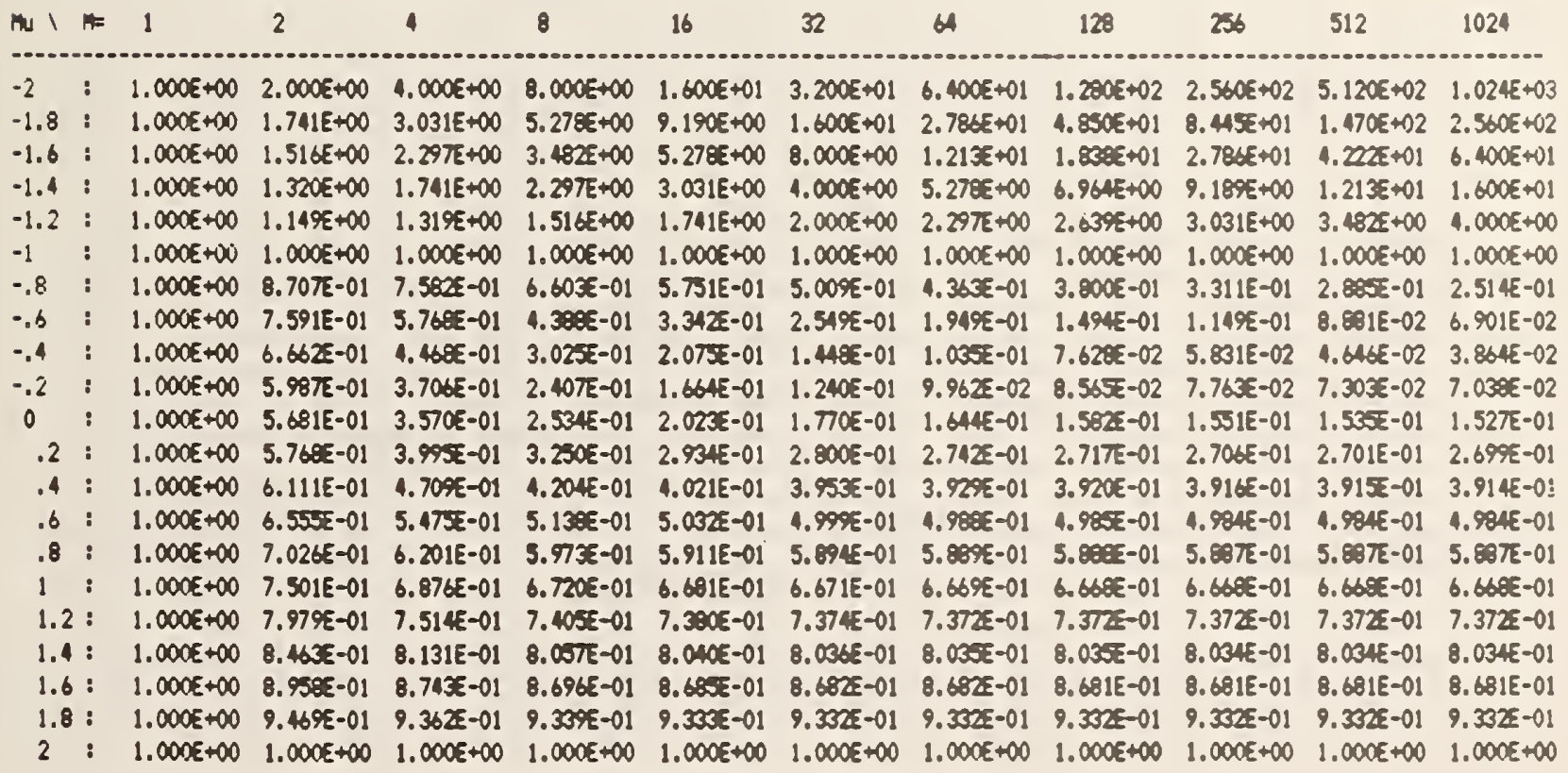


ex(2, $r, r, m)$ for $r=400$

\begin{tabular}{|c|c|c|c|c|c|c|c|c|c|c|c|c|}
\hline 11 & $m$ & d & 2 & 4 & 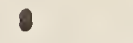 & 16 & 32 & $\mathbf{M}$ & 120 & 25 & 512 & 1024 \\
\hline 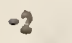 & 8 & $+\infty$ & $+\infty 0$ & $5+\infty$ & $\sum+\infty$ & $5+01$ & $d$ & $c+01$ & $2001+02$ & .02 & $0 \leq+02$ & - 10 \\
\hline-1.8 & 1 & & & $-\mathrm{C}_{2}$ & $2 \pi=+\infty$ & & & ones & $8505+01$ & 0.45E+01 & $0 \leq 402$ & $2.540 E 402$ \\
\hline-1.6 & 1 & $005+00$ & $5+\infty$ & $2 * \pi+\infty$ & 4825100 & $2 \pi=5+\infty$ & 00 & $21 \div \div 01$ & $06 x+01$ & $2.7045+01$ & $200 \div+01$ & $6.400=01$ \\
\hline 1.4 & 8 & $0 \leqslant * 0$ & $30,1+00$ & $1.741 E+00$ & 2. $29 \pi \mathrm{E}+00$ & 3.0S1E+CO & $.0005+00$ & $5.27=3+0$ & $645+00$ & $9.1905+\infty 0$ & $1.213 z+01$ & 1.6005401 \\
\hline-1.2 & $:$ & & $+\infty$ & 1.3 & $+\infty$ & $\infty$ & sow & $+\infty 0$ & $2.63: 15 \div 00$ & $3.001 E+\infty 0$ & $3.4925+00$ & $4.000 E+\infty 0$ \\
\hline 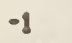 & $:$ & 1.000 & $+\infty$ & $5+\infty$ & 1.00 & $1.0000+00$ & 1.00 & 1.00 & $1.0005+00$ & $1.000 \leqslant+\infty 0$ & $1.000 E+\infty 0$ & $1.000=00$ \\
\hline .8 & 1 & 1. & -01 & & & $5.741:-01$ & -01 & 4.30 & & $3.30<5-01$ & $2.87 \%-01$ & 2.50:5-01 \\
\hline .6 & 8 & & 01 & 1 & & 3.5 & 01 & -01 & 01 & $.12 \%-01$ & $0.45=02$ & -02 \\
\hline .4 & 8 & & 01 & 01 & 01 & 01 & 01 & -02 & & $5.27 \%-02$ & $4.00=-02$ & $3.29 \pi-02$ \\
\hline .2 & 8 & 1.0 & -01 & 3.6 & 2.30 & $1.57=3-08$ & 1.14 & & 02 & $6 . \pi m=-02$ & $6.31 c \varepsilon-02$ & $\xi-02$ \\
\hline 0 & $:$ & $x$ & -01 & 01 & -01 & -01 & & & & & $5-01$ & $=-01$ \\
\hline .2 & 8 & 1.0 & -01 & 3.9 & 3.1 & 2.87 & 2.7 & $=01$ & 01 & $E-01$ & $2.02 \pi 5-01$ & $2.638 \leq-01$ \\
\hline .4 & 1 & 1.00 & 6.09 & 4.68 & {$[-01$} & 3.99 & 3.92 & $3.902-01$ & 3. $925-01$ & 3. $\cos 3-01$ & 3.83TE-01 & $3.837 E-01$ \\
\hline .6 & 8 & $\infty$ & $E-01$ & 3.46 & $5-01$ & 5.02 & $4 . \%$ & 4. $900-01$ & $4.9 \pi / E-01$ & $4.97<5-01$ & $4.975=01$ & $4.9755-01$ \\
\hline .8 & $:$ & & $7.02<=01$ & 6.1 & $5.971 E-01$ & 5. $00:=01$ & 5.69 & $5.68 \pi-01$ & $5.605-01$ & S. $88<\tau-01$ & 5. $8005-01$ & $5.8 \div 5-01$ \\
\hline 1 & $:$ & & $7.500=01$ & 6.87 & $6.719-01$ & $6.6805=01$ & 6.67 & $6.668=01$ & $6.66 \pi=01$ & $6.66 \pi-01$ & $6.66 \pi-01$ & $6.667 E-01$ \\
\hline & 1 & 1.0 & -01 & 7.5 & {$[-01$} & $7.3005=01$ & 7.3 & $7.3725-01$ & -01 & $7.372 x-01$ & $7.372 \leq-01$ & $7.37 z-01$ \\
\hline & $:$ & 1.00 & $0.463 ;-01$ & Q.1 & $8.05 \pi-01$ & 8.04 & $8 . \alpha$ & $8.085 x-01$ & $8.035=01$ & 8.0BLE-01 & $8.0315-01$ & $8.034 E-01$ \\
\hline & 1 & & $0.9585-01$ & 8.74 & $8.696 E-01$ & $8.645-01$ & $8.6825-01$ & $8.6825=01$ & $8.681 E-01$ & 8.681E-01 & $8.681 E-01$ & $8.681 E-01$ \\
\hline 1.1 & $:$ & & $9.46 \%-01$ & & $9.30 \% E-01$ & $9.305 E-01$ & $9.302 E=01$ & $9.3525-01$ & $9.352 E-01$ & $9.332 E-01$ & 9.332E-01 & $9.3325-01$ \\
\hline 2 & 1 & $1.000 E+\infty 0$ & $1.000 E+\infty 0$ & $1.000 E+00$ & $1.000 E+\infty 0$ & $1.0005+\infty$ & $1.000 E+\infty 0$ & $1.000=+\infty 0$ & $1.000 E+\infty 0$ & $1.000 E+\infty 0$ & $1.000 E+\infty 0$ & $1.000 E+00$ \\
\hline
\end{tabular}

$83(2, H, r$, as $)$ for $r=8192$

\begin{tabular}{|c|c|c|c|c|c|c|c|c|c|c|c|c|}
\hline 1 & $h^{3}$ & 1 & 2 & 4 & 8 & 16 & 32 & 64 & 128 & 256 & 512 & 1024 \\
\hline-2 & $:$ & $100 E+\infty 0$ & $000 E+\infty 0$ & $000 \varepsilon+\infty 0$ & $00 \varepsilon+\infty$ & $D O E+01$ & $E+01$ & 01 & $20 E+02$ & $60 \varepsilon+02$ & 02 & , \\
\hline .8 & 1 & $0 E+\infty 0$ & $+\infty$ & $+\infty$ & $+\infty$ & $+\infty$ & 01 & $d$ & $50 E+01$ & & & $E+02$ \\
\hline 1.6 & $:$ & $1.000 E+00$ & $1.516 E+\infty 0$ & $2.29 \pi t+\infty$ & $32 \varepsilon+\infty 0$ & $278 E+\infty 0$ & $.000 E+\infty 0$ & $.213 E+01$ & $8305+01$ & $7865+01$ & $222 x+01$ & $400 E+01$ \\
\hline 1.4 & : & $1.000 E+00$ & $E+\infty$ & 1.741 & $2.297 E+\infty 0$ & $.031 E+\infty 0$ & $4.000 E+00$ & $5.278 E+\infty 0$ & $.964 E+00$ & $.190 E+00$ & 1.213 & $.600 E+01$ \\
\hline 1.2 & $:$ & $1.000 E+\infty 0$ & 100 & $+\infty$ & 100 & $E+\infty$ & $E+\infty 0$ & $2 . x$ & $639+00$ & $31 E+\infty 0$ & $\infty$ & $.000 E+00$ \\
\hline & : & $1.000 E+00$ & 1.0 & $+\infty$ & +00 & $E+\infty$ & $E+\infty$ & 1.00 & $1.0005+00$ & $.000 E+\infty$ & 1.000 & $1.000 E+00$ \\
\hline .8 & : & $1.000 E+\infty 00$ & 8.70 & 7. $500 \varepsilon-01$ & -01 & $5.7465-01$ & 5.00 & $4.3565-01$ & $3.7935-01$ & $3.3035-01$ & $2.876 E-01$ & 2. $505 E-01$ \\
\hline .6 & $:$ & $+\infty$ & & $=01$ & & 01 & 01 & 01 & $461 E-01$ & $5 E-01$ & -02 & $E-02$ \\
\hline .4 & : & $=+\infty$ & 6.6 & $=01$ & 01 & -01 & 1.3 & 9.4 & $6.68 x=02$ & $4.864 E-02$ & .02 & $2.871 E-02$ \\
\hline. .2 & $:$ & $1.000 E+00$ & 5.9 & -01 & 01 & -01 & 1.0 & 8.2 & $6.778 \varepsilon=02$ & $955 x-02$ & $4835-02$ & $5.212-02$ \\
\hline 0 & : & 00 & 5.5 & -01 & & -01 & .01 & -01 & $1.385 z=01$ & $1.351 E-01$ & $.33 x-01$ & $.327 E-01$ \\
\hline .2 & : & 1.00 & 5.7 & -01 & & 2.81 & 2.6 & 2.62 & $2.5945-01$ & 2. 583E-01 & $2.578 E-01$ & $2.576 \varepsilon-01$ \\
\hline .4 & : & 1.00 & 6.00 & 4.66 & 01 & $3.974 E-01$ & 3.9 & $3.881 E-01$ & $3.87 x \leq-01$ & $3.868 \varepsilon-01$ & $3.86 \pi-01$ & $3.86 \pi-01$ \\
\hline .6 & $:$ & $1.000 E+\infty 0$ & 6.54 & $5.4635-01$ & -01 & $5.018 E-01$ & 4.98 & $4.975 E-01$ & $4.971 E-01$ & $4.970 E-01$ & $4.970 E-01$ & $.970 E-01$ \\
\hline .8 & : & $+\infty 0$ & 7.02 & 6. $196 E-01$ & & 5.90 & 5.8 & 5.60 & 5. 884E-01 & 5. & 5.8Q4E-01 & $5.8845-01$ \\
\hline 1 & : & $1.000 E+00$ & 7.500 & $6.875 E-01$ & 6.7 & $6.680 E-01$ & 6.67 & 6.66 & $667 E-01$ & $6.667 E-01$ & -01 & $6.667 E-01$ \\
\hline 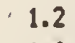 & $:$ & & 7.9 & -01 & 01 & -01 & 01 & -01 & $2 \varepsilon-01$ & $7.37 z-01$ & $172 \leq-01$ & $x-01$ \\
\hline 1.4 & : & 1.00 & 8.46 & $8.131 E-01$ & 8.0 & 8. 040E-01 & 8. $036 E-01$ & $8.035 z-01$ & $8.034 E-01$ & 8.034E-01 & $8.034 E-01$ & $8.03 A E-01$ \\
\hline 1.6 & : & $1.000 E+\infty 0$ & $8.9585-01$ & $8.743 E-01$ & 8.69 & $8.685 \bar{x}-01$ & $8.6825-01$ & $8.682 E-01$ & 8.681E-01 & $8.681 E-01$ & 8.681E-01 & 8. $681 E-01$ \\
\hline 18 & : & & 9.46 & & & & & & $9.332 \leq-01$ & $9.332 \leq-01$ & $9.332 E-01$ & $9.332 E-01$ \\
\hline 2 & : & $1.000 \varepsilon+\infty 0$ & $1.000 E+\infty 0$ & $1.000 E+00$ & $1.000 E+00$ & $1.000 E+00$ & $1.000=+00$ & $1.000 E+\infty 0$ & $1.000 E+00$ & $1.000 E+\infty 0$ & $.000 \varepsilon+\infty$ & $\div+\infty$ \\
\hline
\end{tabular}




\begin{tabular}{|c|c|c|}
\hline \multirow[t]{3}{*}{$\begin{array}{l}\text { NIST-114A } \\
\text { (REV. 3-89) }\end{array}$} & \multirow{3}{*}{$\begin{array}{l}\text { U.S. DEPARTMENT OF COMMERCE } \\
\text { NATIONAL INSTITUTE OF STANDARDS AND TECHNOLOGY } \\
\text { BIBLIOGRAPHIC DATA SHEET }\end{array}$} & $\begin{array}{l}\text { 1. PUBLCATION OR REPORT NUMBER } \\
\text { NIST/TN-1318 }\end{array}$ \\
\hline & & 2. PERFORMING ORGANIZATION REPORT NUMBER \\
\hline & & $\begin{array}{r}\text { 3. PUBLCATION DATE } \\
\text { March } 1990\end{array}$ \\
\hline
\end{tabular}

4. TITLE AND SUBTITLE

Variances Based on Data With Dead Time Between the Measurements

5. AUTHOR(S)

James A. Barnes and David W. Allan

6. PERFORMING ORGANIZATION (IF JOINT OR OTHER THAN NIST, SEE INSTRUCTIONS)

U.S. DEPARTMENT OF COMMERCE

NATIONAL INSTITUTE OF STANDARDS AND TECHNOLOGY

GAITHERSBURG, MD 20899

\begin{tabular}{l} 
7. CONTRACT/ORANT NUMBER \\
\hline 8. TYPE OF REPORT AND PERIOD COVERED
\end{tabular}

9. SPONSORING ORGANIZATION NAME AND COMPLETE ADDRESS (STAEET, CITY, STATE, ZIP)

10. SUPPLEMENTARY NOTES

DOCUMENT DESCRIBES A COMPUTER PROQRAM; SF-185, FIPS SOFTWARE SUMMARY, IS ATTACHED.

11. ABSTRACT (A 200-WORD OR LESS FACTUAL SUMMARY OF MOST SIGNIFICANT INFORMATION. IF DOCUMENT INCLUDES A SIGNIFICANT BIBLIOQRAPHY OR UTERATURE SURVEY, MENTION IT HERE.)

The accepted definition of frequency stability in the time domain is the two-sample variance (or Allan variance). It is based on the measurement of average frequencies over adjacent time intervals, with no "dead time" between the intervals. The primary advantages of the Allan variance are that ( 1 ) it is convergent for many encountered noise models for which the conventional variance is divergent; (2) it can distinguish between many important and different spectral noise types; (3) the two-sample approach relates to many practical implementations, for example, the rms change of an oscillator's frequency from one period to the next; and (4) Allan variances can be easily estimated at integer multiples of the sample interval.

In 1974 a table of bias functions which related variance estimates with various confiqurations of number of samples and dead time to the two-sample (or Allan) variance was published [I]. The tables were based on noises with pure power-law spectral densities.

Often situations occur which unavoidabiy have distributed dead time between measurements, but still the conventional variances are not convergent. Some of these applications are outside of the time and frequency field. Also, the dead times are often distributed throughout a given average, and this distributed dead time is not treated in the 1974 tables.

This paper reviews the bias functions $B_{1}(N, r, \mu)$, and $B_{2}(r, \mu)$ and introduces a new bias function, $B_{3}$ $(2, r, \mu)$, to handle the commonly occurring cases of the effect of distributed dead time on the computed variances. Some convenient and easy to interpret asymptotic limits are reported. A set of tables for the bias functions are included in the Appendix of this paper.

12. KEY WORDS (6TO 12 ENTRIES: ALPHABETICAL ORDER: CAPITALIZ ONLY PROPER NAMES; AND SEPARATE KEY WORDS BY SEMICOLONS) Allan variance; bias functions; data sampling and dead time; dead time between the measurement; definition of frequency stability; distributed dead time; two-sample variance

13. AVAILABIUTY

\section{UNUMITED}

$\mathrm{X}$

$\mathrm{X}$

FOR OFFICIAL DISTRIBUTION. DO NOT RELEASE TO NATIONAL TECHNICAL INFORMATION SEAVICE (NTIS).

ORDER FROM SUPERINTENDENT OF DOCUMENTS, U.S. GOVERMMENT PRINTING OFFICE, WASHINGTON, DC 20402.

ORDER FROM NATIONAL TECHNICAL INFORMATION SERVICE (NTIS), SPRINGFIELD, VA 22161.

14. NUMBER OF PRINTED PAGES

15. PRICE 





\section{NIST Technical Publications}

\section{Periodical}

Journal of Research of the National Institute of Standards and Technology-Reports NIST research and development in those disciplines of the physical and engineering sciences in which the Institute is active. These include physics, chemistry, engineering, mathematics, and computer sciences. Papers cover broad range of subjects, with major emphasis on measurement methodology and the basic technology underlying standardization. Also included from time to time are survey articles on topics closely releted to the Institute's tochnical and scientific programs. Issued six times a year.

\section{Nonperiodicals}

Monograph-Major contributions to the technical literature on various subjects related to the Institute's scientific and technical activities.

Handbooke-Recommended codes of engineering and industrial practice (including safety codes) developed in cooperation with interested industries, professional organizations, and regulatory bodies. Special Publications-Include proceedings of conferences sponsored by NIST, NIST annual reports, and other special publications appropriate to this grouping such as wall charts, pocket cards, and bibliographien.

Applled Mathematics Series-Mathematical tables, manuals, and studies of special interest to physicists, engineers, chemists, biologists, mathematicians, computer programmers, and others engaged in ecientific and technical work.

National Standard Reference Dat Series-Provides quantitative dats on the physical and chemical properties of materials, compiled from the world's literature and critically evaluated. Developed under a worldwide program coordinated by NIST under the authority of the National Standard Data Act (Public Law 90.390). NOTE: The Journal of Physical and Chemical Reference Data (JPCRD) is published quarterly for NIST by the American Chemical Society (ACS) and the American Institule of Phyaics (AIP). Subscriptions, reprints, and supplements are available from ACS, 1155 Sir. teenth St., NW., Washington, DC 20056.

Bulling Selence Sertes-Disceminates technical information developed at the Institute on building materials, components, systems, and whole structures. The series presents research results, teat. methods, and performance criteria related to the structural and environmental functions and the durability and safety characteristics of building elements and systems.

Technical Notes-Studies or reports which are complete in themselves but restrictive in their treatment of a subject. Analogous to monographs but not 20 comprehensive in scope or definitive in treatment of the subject aren. Often serve as a vehicle for final reports of work performed at NIST under the sponsorihnip of other government agenciea.

Voluntur Produet Standaris-Developed under procedures published by the Department of Commerce in Part 10, Title 15, of the Code of Federal Regulations. The atandards extablish nationally recognized requirements for products, and provide all concerned interests with a basis for common undentanding of the charecteristics of the products. NIST administers this program as a supplement to the ectivities of the private sector standerdizing organizations.

Consumer Information Series-Practical information, based on NIST resenrch and experience, covering areas of interest to the consumer. Easily understandable language and illustrations provide useful buckground knowledge for shopping in today's technological marketplece.

Order the above NIST publications from: Superintendens of Documents, Covernment Printing Office, Washingram, DC 20102

Onder the following NIST publications-FIPS and NISTIRs-from the National Technical Information Service, Springield, VA 22161.

Pederal Inforation Procesoling Standards Publications (RIPS PUB)-Publications in this series collectively comstitute the Federal Information Processing Standards Register. The Register serves as the official cource of information in the Redert Government regarding standards issued by NIST pursuant to the Federal Property and Administrative Services Act of 1949 as amended, Public Law 89.306 (79 Stat. 1127), and as implemented by Executive Order 11717 (38 FR 12315, dated May 11, 1973) and Part 6 of Title 15 CFR (Code of Foderl Regulations).

NIST Intentemey Reports (NISTLR) - A special series of interim or find reports on work performed by NIST for outside eponson (both government and non-government). In general, initial distributon b hadied by the aponsor, public distribution is by the Nationd Technical Information Service, Springfield, VA 22161, in paper copy or microfiche form. 
U.8. DEPARTMENT OF COMMERCE

Natlonal Institute of Standards and Tochnology

(formerty National Bureau of Standards)

325 Broadway

Boulder, Colorado 803033328

\section{OFFICIAL BUSINESS}

PENALTY FOR PRIVATE USE, $\$ 300$ 